\title{
Theory of Disclinations: IV. Straight Disclinations
}

\author{
Roland deWit
}

Institute for Materials Research, National Bureau of Standards, Washington, D.C. 20234

(May 31, 1973)

\begin{abstract}
The general theory of disclinations developed earlier is applied to the special case of a straight disclination line. First the geometrical fields are found, such as the defect loop densities which correspond to Mura's new concepts of "plastic distortion" and "plastic rotation," the basic plastic fields (strain and bend-twist), the defect densities (dislocation and disclination), the characteristic vectors (Burgers and Frank), and the incompatibility. Then the static fields are found for the isotropic case, such as the displacement, total distortion, basic elastic fields, and the stress. It is shown that the disclination axis is moved by adding a dislocation to the disclination line. All these special results for the straight disclination line are shown to satisfy the general equations of the theory. As corollaries the following topics are also treated:

1. The finite and infinitesimal straight disclination dipole, which can be biaxial or uniaxial. It resembles the straight dislocation line.

2. The dislocation models of the straight disclination line and of the finite disclination dipole. They are terminating dislocation walls (tilt and twist).

3. The compensated disclination line and the bent dislocation wall.

4. Finally we show analytically a special case of a dislocation ending on a disclination.
\end{abstract}

Key words: Burgers vector; continuum mechanics; defect; dipole; disclination; dislocation; distortion; Green's tensor; incompatibility; loop; plasticity; strain; Volterra.

\section{Contents}

1. Introduction.

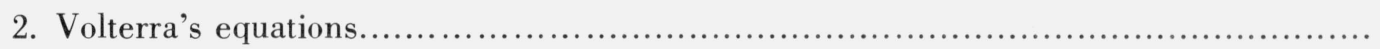

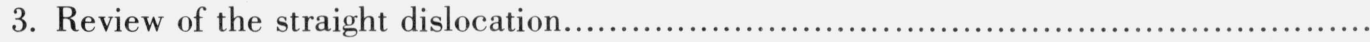

3.1 Geometry....

3.2 Isotropic statics

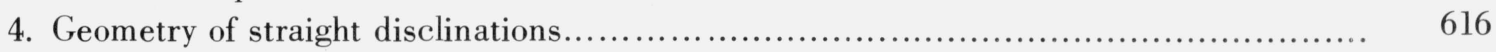

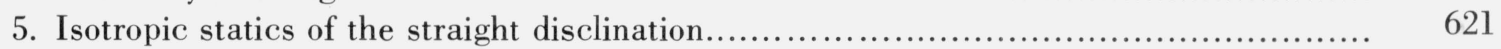

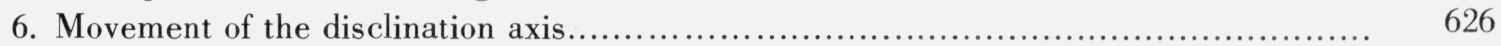

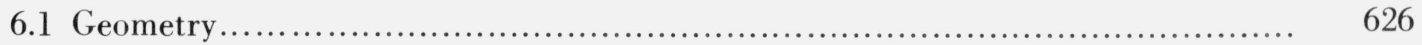

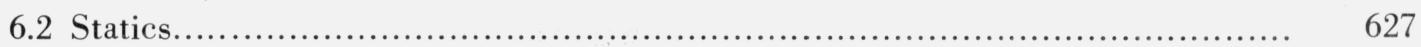

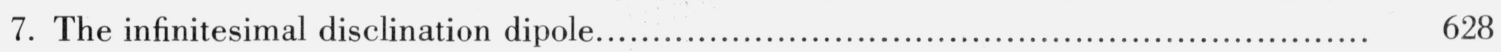

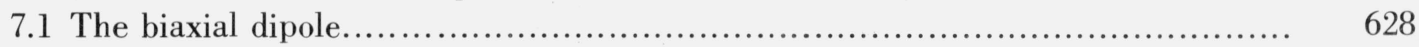

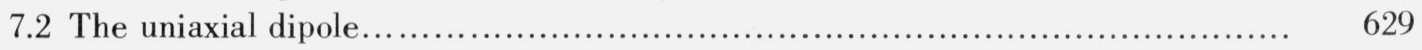

8. The "Dislocation model" of the straight disclination................................ 631

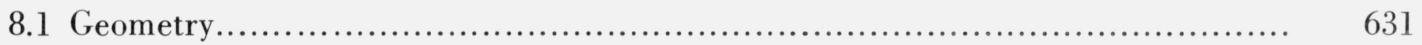

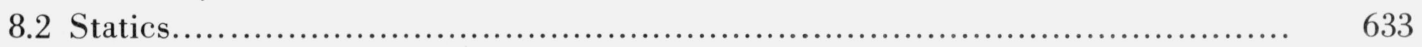

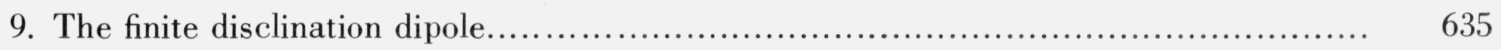

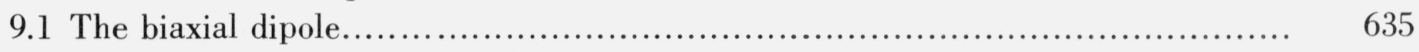

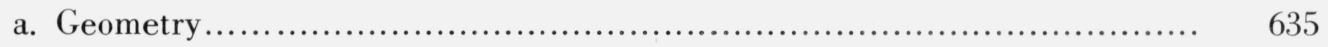

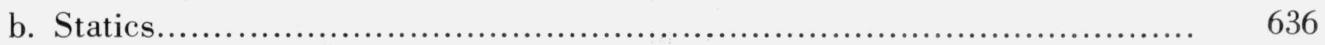

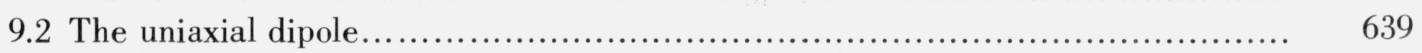


10. The compensated disclination line

11. The bent dislocation wall.

12. Example of a terminating dislocation line.

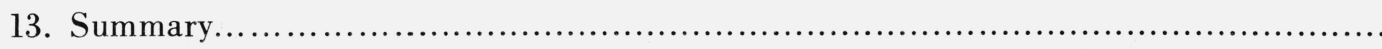

14. Appendix A. Evaluation of the delta function on a straight line and a semi-infinite

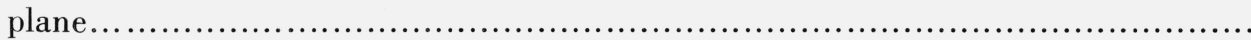

15. Appendix B. Generalized derivatives of cylindrical coordinates.

16. Appendix C. Derivation of static fields of a straight defect from the general theory......

17. References

\section{Introduction}

In previous papers $[1,2,3]^{1}$ we have developed the general linear theory of stationary disclinations in an anisotropic or isotropic body. In the present paper we wish to specialize some of these results to the case of discrete straight disclination lines.

In 1907 Volterra [4] gave the elastic fields of straight hollow core dislocations and disclinations running along the axis of a long isotropic cylinder. He called these defects "distortions." Later Love [5] ventured to call them "dislocations." This name has been widely accepted, but its meaning has been narrowed to translational dislocations, because of the early importance of this type of defect in plastic deformation. Therefore Frank [6] introduced the new name "disinclination," later changed to disclination, to describe the rotational dislocation. Furthermore the word "distortion" has since come to stand for the gradient of the displacement, and it would therefore lead to confusion if we still tried to ascribe to it Volterra's original meaning as the combination of dislocations and disclinations. Still it would be useful to have a name for this combination, and we have adopted the word defect for that purpose. In this terminology, then, the present paper deals with discrete straight defect lines. This word usage does not exclude the consideration of "point defects," because they can be regarded as combinations of discrete dislocation loops [3].

We would here also like to point out that it has become customary to refer to "disclination theory" when really "defect theory" is meant (i.e., the theory of dislocations and disclinations). We have done this in our paper titles to emphasize the fact that the new results in defect theory are obtained because disclinations have been introduced.

Furthermore there are at least three independent ways of defining the two types of defects, i.e., in terms of (1) continuous distributions, (2) discrete lines, or (3) infinitesimal loops, which lead to essentially different descriptions of the disclination. This means, for example, that a discrete disclination line contains a certain amount of dislocation density, or a certain different amount of dislocation loop density. In this paper we shall identify these quantities for the case of straight disclinations.

In section 2 we recall that Volterra also summarized the characteristic terms in the displacement fields for straight defects. These results have to be slightly modified to obtain equilibrium everywhere.

Section 3 reviews straight dislocations to show the approach used in specializing the general theory. It also serves as a reference for the later sections.

In section 4 we apply the general results of [2] to our special case of discrete straight disclinations. We find the defect loop densities which are Mura's "plastic distortion" and "plastic rotation." Then we identify the basic plastic fields from which all other results follow. We find the defect densities and show that they satisfy the necessary general relations. The Burgers and Frank vectors are examined, and we conclude with relations for the incompatibility tensor.

Section 5 treats the isotropic statics of straight disclinations. From the given displacement we derive the basic elastic fields, and show that they satisfy the field equations with the defect densities. Then we find the stress and show that it maintains equilibrium.

${ }^{1}$ Figures in brackets indicate the literature references at the end of this paper. 
In section 6 we find how the fields are modified when the disclination axis is moved away from the origin. We show that it is equivalent to adding a dislocation to the disclination.

Section 7 treats the infinitesimal straight disclination dipole line.

In section 8 we give the dislocation model of the straight disclination. We show that it is a terminating dislocation wall (in general tilt and twist). It has the same displacement, elastic strain and stress as the corresponding disclination line. In terms of the dislocation model we find more precise interpretations of Mura's new concepts.

Section 9 treats the finite straight disclination dipole line. We show that it reduces to the infinitesimal case of section 7 when the width approaches zero.

In section 10 we derive the results for the straight compensated disclination line, using two different defect surfaces.

In section 11 we apply the results of section 10 to the bent dislocation wall, which is made up of two semi-infinite dislocation walls meeting at right angles.

Section 12 shows analytically how a dislocation can end on a disclination.

Throughout the paper we assume that the body is infinitely extended. The geometric results are valid for the anisotropic case, but we have obtained the static fields only for the isotropic body.

We shall freely refer to the results of the three previous papers [1,2,3], which will be denoted in the present paper as I, II, and III.

\section{Volterra's Equations}

Weingarten's theorem [1, 2] states: On following around an irreducible circuit in a multiplyconnected body, the displacement and rotation change by an amount that represents a rigid body motion, if and only if the classical elastic compatibility conditions are satisfied throughout the body.

This theorem led Volterra to conceive of the defects he called "distortions." They have subsequently also been called the most general Weingarten-Volterra dislocations. Volterra then proceeded to calculate the properties of such straight defects lying along the $z$ axis. He first found the terms in the displacement fields that characterize such defects. Nabarro [7] considered these so important to dislocation theory that he quoted them directly in his book (eq (2.20)):

$$
\begin{aligned}
& 2 \pi u_{x}=\left(b_{x}-d_{z x} z+d_{x y} y\right) \tan ^{-1}(y / x)+\frac{1}{2}\left(-b_{y}-d_{y z} z-\alpha d_{x y} x\right) \ln \left(x^{2}+y^{2}\right), \\
& 2 \pi u_{y}=\left(b_{y}-d_{x y} x+d_{y z} z\right) \tan ^{-1}(y / x)+\frac{1}{2}\left(b_{x}-d_{z x} z-\alpha d_{x y} y\right) \ln \left(x^{2}+y^{2}\right), \\
& 2 \pi u_{z}=\left(b_{z}-d_{y z} y+d_{z x} x\right) \tan ^{-1}(y / x)+\frac{1}{2}\left(d_{y z} x+d_{z x} y\right) \ln \left(x^{2}+y^{2}\right),
\end{aligned}
$$

where $\alpha$ is an elastic constant. These displacements maintain equilibrium under no body forces except at the $z$ axis. It will be recognized that the terms with coefficients $b$ represent possible displacements for dislocations. The terms with coefficients $d$ will be seen to be possible displacements for straight discrete disclinations.

These equations have to be modified to obtain equilibrium under no body forces also at the $z$ axis. This can be done in several ways. One method is to calculate the body force from (2.1) by the usual equations of elasticity. It is found to be singular at the $z$ axis. Then the displacement field of an equal but opposite force along the $z$ axis is derived, and added to (2.1) to get the desired result. We do not show the details in this paper. Another method is to use the general expressions from III. Even though straight defects do not satisfy the necessary boundary conditions used there, it is still possible to find the correct functional dependence of the fields from the general expressions. The details are given in appendix $\mathrm{C}$.

Once a set of displacement functions is found "by hook or crook," it is easy to verify that they are a solution by showing that they maintain equilibrium everywhere, including at singular points, and that they include the characteristic Volterra terms (2.1). Equilibrium is checked simply by deriving the stress and showing that it satisfies the equilibrium equation. 
The development in this paper can also be put in terms of the following general question: Given the displacement as an empirical function of the coordinates representing experimental data, what is the system of defects that would produce such a displacement? To answer this question we have to determine how to divide the resulting fields into elastic and plastic parts. For a discrete defect this will be simple to do, because the most logical candidates for the basic plastic fields are the terms that are singular on a particular surface, as we shall see for the dislocation in section 3.2 and for the disclination in section 5. However, by another division we can also find a different system of defects that will give the same displacement, as we shall also see for the "dislocation model" of the straight disclination in section 8 .

\section{Review of the Straight Dislocation}

In this section we apply the general results of section II $3.2^{*}$ to the special case of a straight dislocation.

\subsection{Geometry}

The plastic distortion for a constant displacement across a surface $S$ and the dislocation density for the corresponding discrete dislocation along the line $L$ are given by the general expressions, (II3.21, II3.23), *

$$
\begin{aligned}
& \beta_{k l}^{P}(\mathbf{r})=-\delta_{k}(S) b_{l}, \\
& \alpha_{p l}(\mathbf{r})=\delta_{p}(L) b_{l},
\end{aligned}
$$

where $L$ is the closed boundary of $S, \delta_{k}(S)$ and $\delta_{p}(L)$ are the Dirac delta functions on the surface $S$ and the curve $L$, respectively, defined by (A11-12) in appendix A, and $b_{l}$ is constant, identified as the Burgers vector of the discrete dislocation line. For the special case of this paper we wish to take $L$ as a straight line and $S$ as a semi-infinite plane bounded by $L$. In particular, let $L$ run along the $x_{3}$ axis and $S$ be the part of the $x_{1} x_{3}$ plane for negative $x_{1}$. To satisfy the right-hand rule we take the normal of $S$ in the negative $x_{2}$ direction. These relationships are illustrated in figure 1 . Then the only nonvanishing components of (3.1-2) become

$$
\begin{gathered}
\beta_{2 l}^{P}=b_{l} H\left(-x_{1}\right) \delta\left(x_{2}\right), \\
\alpha_{3 l}=b_{l} \delta\left(x_{1}\right) \delta\left(x_{2}\right),
\end{gathered}
$$

according to (A15-16). The plastic distortion is singular on the plane $S$, and the dislocation density is concentrated on the $x_{3}$ axis. The three different cases for $l=1,2,3$ are illustrated in figures $2-4$. In figures 2 and 4 the plane $S$ is also the slip plane, because it contains the Burgers vector.

For consistency we see that these results satisfy the definition, (I5.2), (II3.4),

$$
\alpha_{p l} \equiv-\epsilon_{p m k} \beta_{k l, m}^{P},
$$

by (A6). We see also that (3.4) satisfies the continuity condition, (I5.4), (II3.24),

$$
\alpha_{p l, p}=0 .
$$

Now take for a Burgers circuit $\lambda$ a circle of radius $a$ in the $x_{1} x_{2}$ plane centered at the origin (fig. 1).

* Section II 3.2 refers to section 3.2 in reference [2], and the symbol (II3.21) refers to eq (3.21) in reference [2]. 


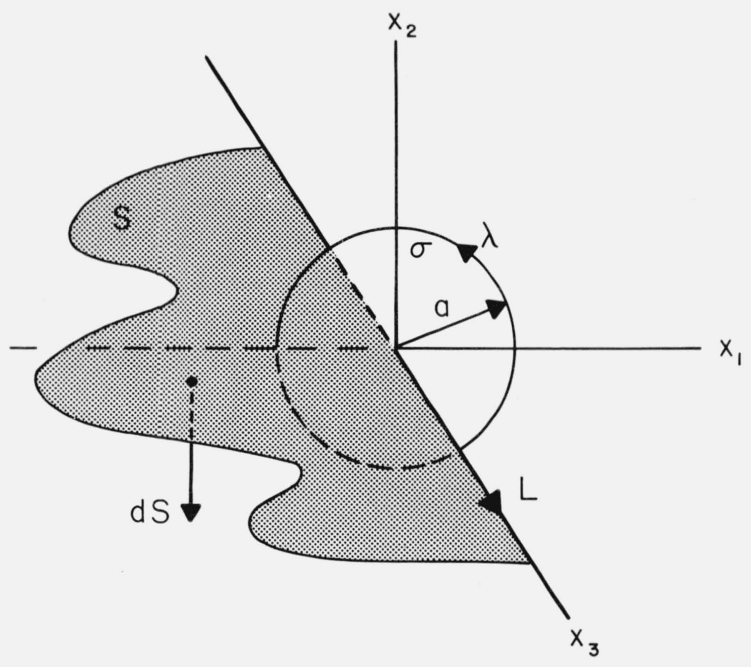

Figure 1. Geometry of the discrete straight defect line in the Cartesian coordinate system $x_{1} x_{2} x_{3}$.

The defect line $L$ is along the $x_{3}$ axis and the defect surface $S$ is the semi-infinite part of the $x_{1} x_{3}$ plane for which $x_{1}$ is a negative, with a normal in the negative $x_{2}$ direction. For the Burgers circuit $\lambda$, which links $L$, and the Burgers surface $\sigma$, which is pierced by $L$, we have chosen a circle of radius $a$ in the $x_{1} x_{2}$ plane centered at the origin.

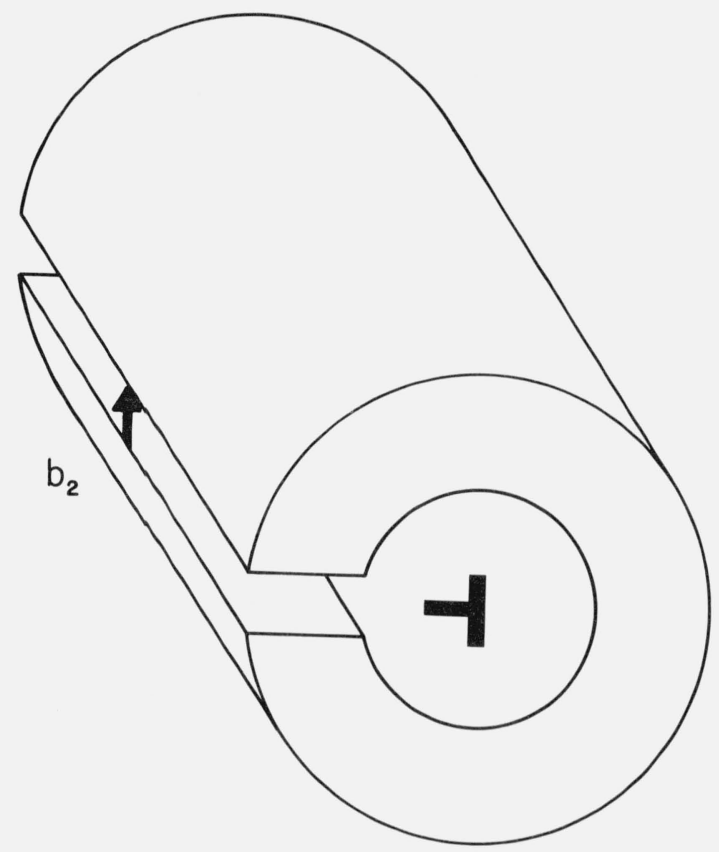

Figure 3. Edge dislocation described by the dislocation density $\alpha_{32}=b_{2} \delta\left(x_{1}\right) \delta\left(x_{2}\right)$

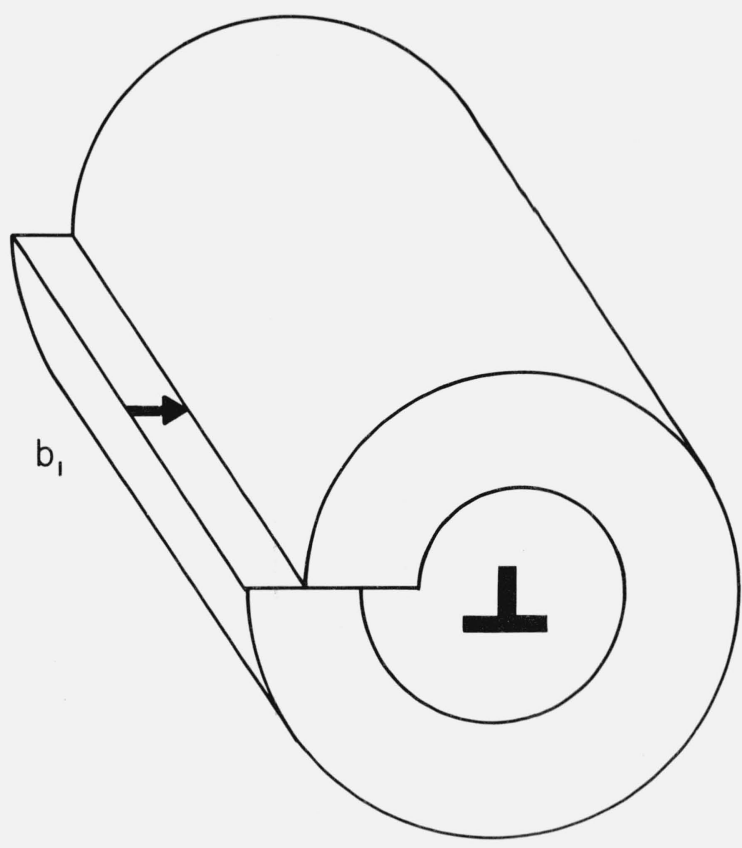

Figure 2. Edge dislocation described by the dislocation density $\alpha_{31}=b_{1} \delta\left(x_{1}\right) \delta\left(x_{2}\right)$.

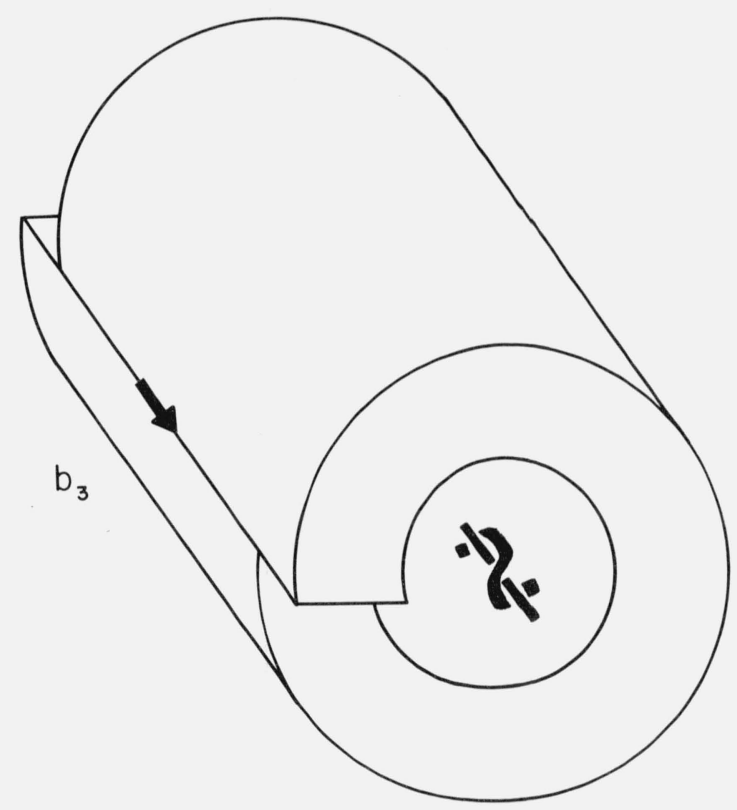

Figure 4. Screw dislocation described by the dislocation density $\alpha_{33}=b_{3} \delta\left(x_{1}\right) \delta\left(x_{2}\right)$. 
Then we have by (3.3) and (A23)

$$
-\oint_{\lambda} \beta_{k l}^{P} d L_{k}=-\oint_{\lambda} b_{l} H\left(-x_{1}\right) \delta\left(x_{2}\right) d L_{2}=b_{l},
$$

in agreement with (II3.22), confirming that $b_{l}$ is the Burgers vector. We also have by (3.4) and (A22)

$$
\int_{\sigma} \alpha_{p l} d S_{p}=\int_{\sigma} b_{l} \delta\left(x_{1}\right) \delta\left(x_{2}\right) d S_{3}=b_{l},
$$

in agreement with (II3.25). So in this section we have found the special forms of the plastic distortion and dislocation density (3.3-4) for a straight dislocation running along the $x_{3}$ axis, and these equations are in complete agreement with all the general results for geometry.

We conclude this section with expressions for the incompatibility tensor. It is in general given by, (I5.3),

$$
\eta_{p q}=-\left(\epsilon_{q n l} \alpha_{p l, n}\right)_{(p q)}
$$

For our special case the only nonvanishing components are

$$
\begin{aligned}
& \eta_{13}=-\frac{1}{2} b_{3} \delta\left(x_{1}\right) \delta^{\prime}\left(x_{2}\right), \\
& \eta_{23}=\frac{1}{2} b_{3} \delta^{\prime}\left(x_{1}\right) \delta\left(x_{2}\right), \\
& \eta_{33}=b_{1} \delta\left(x_{1}\right) \delta^{\prime}\left(x_{2}\right)-b_{2} \delta^{\prime}\left(x_{1}\right) \delta\left(x_{2}\right),
\end{aligned}
$$

from (3.4). We note that they satisfy the continuity condition, (I4.2),

$$
\eta_{p q, p}=0 .
$$

The results in this section apply to an infinitely extended body, whether isotropic or anisotropic.

\subsection{Isotropic Statics}

In this section we find the static fields for a straight dislocation in an infinitely extended isotropic body. The displacement is given by (appendix $\mathrm{C}$ )

$$
\begin{aligned}
& u_{1}^{T}=b_{1}\left[\frac{\varphi}{2 \pi}+\frac{x_{1} x_{2}}{4 \pi(1-\nu) \rho^{2}}\right]+\frac{b_{2}}{4 \pi(1-\nu)}\left[(1-2 \nu) \ln \rho+\frac{x_{2}^{2}}{\rho^{2}}\right], \\
& u_{2}^{T}=-\frac{b_{1}}{4 \pi(1-\nu)}\left[(1-2 \nu) \ln \rho+\frac{x_{1}^{2}}{\rho^{2}}\right]+b_{2}\left[\frac{\varphi}{2 \pi}-\frac{x_{1} x_{2}}{4 \pi(1-\nu) \rho^{2}}\right], \\
& u_{3}^{T}=\frac{b_{3} \varphi}{2 \pi},
\end{aligned}
$$

where $\nu$ is Poisson's ratio. These expressions are well-known for a dislocation line of Burger vector $b_{l}$ running along the $x_{3}$ axis. We have used a cartesian coordinate system $x_{1} x_{2} x_{3}$, as shown in figure 1. The cylindrical coordinates $\rho$ and $\phi$ are defined in appendix B and shown in figure 5. By restricting the angle $\phi$ to the range $(-\pi, \pi)$ it becomes discontinuous on the semi-infinite plane $S$ shown in figure 1, which then becomes the natural plastic displacement surface of section 3.1. Note that we have labeled (3.12) as the total displacement. In the literature these expressions are 


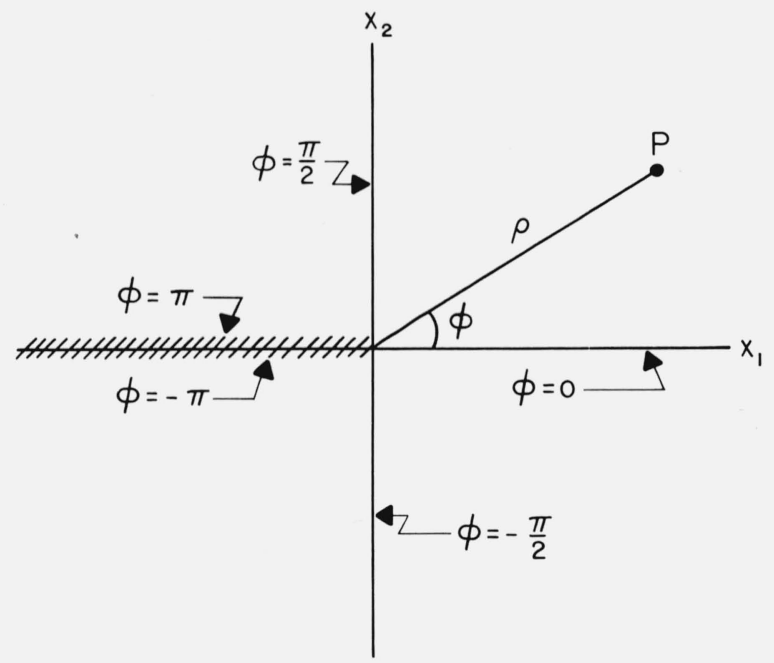

Figure 5. Relation between Cartesian coordinates $\mathrm{x}_{1}$ and $\mathrm{x}_{2}$ and cylindrical coordinates $\rho$ and $\phi$.

The angle $\phi$ has the range $(-\pi, \pi)$, assumes the values shown at the ordinates, and has a jump of $2 \pi$ across the negative $x_{1}$ axis.

usually regarded as the elastic displacement which is then taken to be multiple-valued on account of the angle $\phi$. In the framework of the present theory, however, it would not be correct to regard (3.12) as the elastic displacement. For, if the elastic displacement is prescribed, then there can be no dislocations (c.f. I2.1, I2.2, and I5.6, c.f. also footnote 10 in II). But if the total displacement is given the dislocations are still unspecified. We see from (3.5) that they are specified when we determine how the total distortion is split into an elastic and a plastic part. Intuitively we can see that it is unreasonable to split the displacement into an elastic and a plastic part in the present special case because of the fact that the angle $\phi$ cannot be split into a continuous and a discrete part. In general we shall contend that if a total field quantity of a straight defect contains the angle $\phi$, it is unreasonable to split it into an elastic and plastic part. This idea will also be applied in section 5. The results (3.12) show a logarithmic dependence for the displacement at large distances from the dislocation line, in agreement with table II3.

The total distortion is defined in terms of the total displacement and consists of an elastic and plastic part, (I2.1), (II3.9),

$$
\beta_{k l}^{T} \equiv u_{l, k}^{T}=\beta_{k l}+\beta_{k l}^{P} .
$$

On differentiating the displacement (3.12), the terms containing $\phi$ will produce expressions singular on $S$, by (B4). These expressions correspond exactly to the plastic distortion (3.3) found in the previous section. This apparent coincidence is of course achieved by our special choice of $S$ and the range of $\phi$. These terms occur only because we chose the angle $\phi$ to be discontinuous rather than multiple-valued. Alternatively, if we had no a priori knowledge of the plastic distortion, we could assign the singular terms to it. These terms intuitively represent a surface along which a plastic displacement has taken place, as discussed in section 3.1. So we find for the elastic distortion of the straight dislocation

$$
\beta_{11}=-\frac{b_{1}}{4 \pi(1-\nu)}\left[(1-2 \nu) \frac{x_{2}}{\rho^{4}}+2 \frac{x_{1}^{2} x_{2}}{\rho^{4}}\right]+\frac{b_{2}}{4 \pi(1-\nu)}\left[(1-2 \nu) \frac{x_{1}}{\rho^{2}}-\frac{x_{1} x_{2}^{2}}{\rho^{4}}\right],
$$




$$
\begin{aligned}
& \beta_{21}=\frac{b_{1}}{4 \pi(1-\nu)}\left[(3-2 \nu) \frac{x_{1}}{\rho^{2}}-2 \frac{x_{1} x_{2}^{2}}{\rho^{4}}\right]+\frac{b_{2}}{4 \pi(1-\nu)}\left[(1-2 \nu) \frac{x_{2}}{\rho^{2}}+2 \frac{x_{1}^{2} x_{2}}{\rho^{4}}\right], \\
& \beta_{31}=0, \\
& \beta_{12}=-\frac{b_{1}}{4 \pi(1-\nu)}\left[(1-2 \nu) \frac{x_{1}}{\rho^{2}}+2 \frac{x_{1} x_{2}^{2}}{\rho^{4}}\right]-\frac{b_{2}}{4 \pi(1-\nu)}\left[(3-2 \nu) \frac{x_{2}}{\rho^{2}}-2 \frac{x_{1}^{2} x_{2}}{\rho^{4}}\right], \\
& \beta_{22}=-\frac{b_{1}}{4 \pi(1-\nu)}\left[(1-2 \nu) \frac{x_{2}}{\rho^{2}}-2 \frac{x_{1}^{2} x_{2}}{\rho^{4}}\right]+\frac{b_{2}}{4 \pi(1-\nu)}\left[(1-2 \nu) \frac{x_{1}}{\rho^{2}}+2 \frac{x_{1} x_{2}^{2}}{\rho^{4}}\right], \\
& \beta_{32}=0, \\
& \beta_{13}=-\frac{b_{3} x_{2}}{2 \pi \rho^{2}} \\
& \beta_{33}=0, \\
& \beta_{23}=\frac{b_{3} x_{1}}{2 \pi \rho^{2}} \\
&
\end{aligned}
$$

using (B3-7). By contrast with the plastic distortion (3.3), we see that the elastic distortion consists of functions that are continuous everywhere except at the $x_{3}$ axis. This is reasonable to expect for an elastic field. To check the consistency of our results, it is easily shown that the elastic distortion and the dislocations density (3.4) satisfy the field equation, (I5.6), (II3.10),

$$
\epsilon_{p m k} \beta_{k l, m}=\alpha_{p l},
$$

by (B8-10). This result then confirms that (3.14) represents the elastic distortion of a discrete dislocation line running along the $x_{3}$ axis.

Next we want to show that equilibrium is maintained everywhere. We first find the elastic strain, which is in general given by, (II3.13),

$$
e_{k l}=\beta_{(k l)}
$$

Hence we have from (3.14) for the elastic strain of a straight dislocation

$$
\begin{aligned}
& e_{11}=-\frac{b_{1}}{4 \pi(1-\nu)}\left[(1-2 \nu) \frac{x_{2}}{\rho^{2}}+2 \frac{x_{1}^{2} x_{2}}{\rho^{4}}\right]+\frac{b_{2}}{4 \pi(1-\nu)}\left[(1-2 \nu) \frac{x_{1}}{\rho^{2}}-2 \frac{x_{1} x_{2}^{2}}{\rho^{4}}\right], \\
& e_{22}=-\frac{b_{1}}{4 \pi(1-\nu)}\left[(1-2 \nu) \frac{x_{2}}{\rho^{2}}-2 \frac{x_{1}^{2} x_{2}}{\rho^{4}}\right]+\frac{b_{2}}{4 \pi(1-\nu)}\left[(1-2 \nu) \frac{x_{1}}{\rho^{2}}+2 \frac{x_{1} x_{2}^{2}}{\rho^{4}}\right], \\
& e_{33}=0
\end{aligned}
$$


$e_{12}=\frac{b_{1}}{4 \pi(1-\nu)}\left[\frac{x_{1}}{\rho^{2}}-2 \frac{x_{1} x_{2}^{2}}{\rho^{4}}\right]-\frac{b_{2}}{4 \pi(1-\nu)}\left[\frac{x_{2}}{\rho^{2}}-2 \frac{{ }_{1}^{2} x_{2}}{\rho^{4}}\right]$

$e_{23}=\frac{b_{3} x_{1}}{4 \pi \rho^{2}}$

$e_{31}=-\frac{b_{3} x_{2}}{4 \pi \rho^{2}}$

The dilatation $e=e_{k k}$ is

$$
e=-\frac{1-2 \nu}{2 \pi(1-\nu) \rho^{2}}\left(b_{1} x_{2}-b_{2} x_{1}\right)
$$

At large distances from the dislocation line the elastic strain varies inversely with the distance, in agreement with table II3. Hooke's law for an isotropic body is in general given by, (III3.2),

$$
\sigma_{k l}=2 G\left(e_{k l}+\frac{\nu}{1-2 \nu} e \delta_{k l}\right)
$$

Hence we find for the stress of the straight dislocation

$$
\begin{aligned}
& \sigma_{11}=-\frac{G b_{1}}{2 \pi(1-\nu)}\left[\frac{x_{2}}{\rho^{2}}+2 \frac{x_{1}^{2} x_{2}}{\rho^{4}}\right]+\frac{G b_{2}}{2 \pi(1-\nu)}\left[\frac{x_{1}}{\rho^{2}}-2 \frac{x_{1} x_{2}^{2}}{\rho^{4}}\right], \\
& \sigma_{22}=-\frac{G b_{1}}{2 \pi(1-\nu)}\left[\frac{x_{2}}{\rho^{2}}-2 \frac{x_{1}^{2} x_{2}}{\rho^{4}}\right]+\frac{G b_{2}}{2 \pi(1-\nu)}\left[\frac{x_{1}}{\rho^{2}}+2 \frac{x_{1} x_{2}^{2}}{\rho^{4}}\right], \\
& \sigma_{33}=-\frac{G \nu}{2 \pi(1-\nu)}\left(2 \frac{b_{1} x_{2}}{\rho^{2}}-2 \frac{b_{2} x_{1}}{\rho^{2}}\right), \\
& \sigma_{12}=\frac{G b_{1}}{2 \pi(1-\nu)}\left[\frac{x_{1}}{\rho^{2}}-2 \frac{x_{1} x_{2}^{2}}{\rho^{4}}\right]-\frac{G b_{2}}{2 \pi(1-\nu)}\left[\frac{x_{2}}{\rho^{2}}-2 \frac{x_{1}^{2} x_{2}}{\rho^{4}}\right], \\
& \sigma_{23}=\frac{G b_{3} x_{1}}{2 \pi \rho^{2}}, \\
& \sigma_{31}=-\frac{G b_{3} x_{2}}{2 \pi \rho^{2}} .
\end{aligned}
$$

These expressions are also well-known in the literature. Finally, the equation of equilibrium are, (II2.1),

$$
f_{l}=-\sigma_{k l, k}
$$

where $f_{l}$ is the body force per unit volume. From (3.19) and (B8-10) we find

$$
f_{l}=0
$$

everywhere, including at the $x_{3}$ axis where (3.19) is singular. So the above fields maintain equilib- 
rium without body forces, including at the $x_{3}$ axis. The displacement (3.12) contains the characteristic Volterra terms (2.1) for a dislocation. Therefore, as discussed in section 2, we may conclude that (3.12) correctly represents the displacement of a dislocation.

We conclude this section with expressions for the elastic rotation of the dislocation, which is in general given by (III4.6),

$$
\omega_{q}=\frac{1}{2} \epsilon_{k l q} \beta_{k l},
$$

or, from (3.14), for our special case by

$$
\begin{aligned}
& \omega_{1}=\frac{b_{3} x_{1}}{4 \pi \rho^{2}}, \\
& \omega_{2}=\frac{b_{3} x_{2}}{4 \pi \rho^{2}}, \\
& \omega_{3}=-\frac{b_{1} x_{1}}{2 \pi \rho^{2}}-\frac{b_{2} x_{2}}{2 \pi \rho^{2}} .
\end{aligned}
$$

In this section we have summarized the well-known expressions for the displacement and the elastic fields of a straight dislocation in an infinite isotropic body.

\section{Geometry of Straight Disclinations}

In this section we shall apply the general geometrical results of section II5.2 to the special case of a straight disclination. For a discrete defect along the line $L$, which has been formed by plastic displacement across the surface $S$, the dislocation and disclination loop densities are in general given by, (II5.12-13),

$$
\begin{aligned}
& \beta_{k l}^{*}(\mathbf{r})=-\delta_{k}(S)\left\{b_{l}+\epsilon_{l q r} \Omega_{q}\left(x_{r}-x_{r}^{0}\right)\right\}, \\
& \varphi_{k q}^{*}(\mathbf{r})=-\delta_{k}(S) \Omega_{q},
\end{aligned}
$$

where $b_{l}$ is the dislocation Burgers vector, $\Omega_{q}$ the Frank vector, $x_{r}^{0}$ a point through which the disclination axis passes, and $\delta_{k}(S)$ is the Dirac delta function on the surface $S$, defined by (A12) in appendix A. The quantities (4.1-2) were introduced by Mura [9], who called them the "plastic distortion" and "plastic rotation." For our special case of a straight disclination running along the $x_{3}$ axis, we take $S$ to be the semi-infinite $x_{1} x_{3}$ plane for negative $x_{1}$ with normal in the negative $x_{2}$ direction, as illustrated in figure 1 , and set $b_{l}=0$. Furthermore, for simplicity at this point, we let the axis of rotation pass through the origin, so that $x_{r}^{0}=0$. The effect of moving the axis will be investigated in section 6 . Then the only nonvanishing components of $(4.1-2)$ become

$$
\begin{aligned}
& \beta_{2 l}^{*}=\epsilon_{l q r} \Omega_{q} x_{r} H\left(-x_{1}\right) \delta\left(x_{2}\right), \\
& \varphi_{2 q}^{*}=\Omega_{q} H\left(-x_{1}\right) \delta\left(x_{2}\right),
\end{aligned}
$$

according to (A16). For convenience, we write these results out in full

$$
\begin{aligned}
& \beta_{21}^{*}=\Omega_{2} x_{3} H\left(-x_{1}\right) \delta\left(x_{2}\right), \\
& \beta_{22}^{*}=\left(\Omega_{3} x_{1}-\Omega_{1} x_{3}\right) H\left(-x_{1}\right) \delta\left(x_{2}\right), \\
& \beta_{23}^{*}=-\Omega_{2} x_{1} H\left(-x_{1}\right) \delta\left(x_{2}\right),
\end{aligned}
$$




$$
\begin{aligned}
& \varphi_{21}^{*}=\Omega_{1} H\left(-x_{1}\right) \delta\left(x_{2}\right), \\
& \varphi_{22}^{*}=\Omega_{2} H\left(-x_{1}\right) \delta\left(x_{2}\right), \\
& \varphi_{23}^{*}=\Omega_{3} H\left(-x_{1}\right) \delta\left(x_{2}\right),
\end{aligned}
$$

where we have used (A8). The basic plastic fields (plastic strain and bend-twist) are in general given by, (II5.14-15),

$$
\begin{aligned}
e_{k l}^{P} & =\beta_{(k l)}^{*}, \\
\kappa_{m q}^{P} & =\frac{1}{2} \epsilon_{k l q} \beta_{k l, m}^{*}+\varphi_{m q}^{*} .
\end{aligned}
$$

For our special case we easily find the only nonvanishing components for the plastic strain

$$
\begin{aligned}
& e_{12}^{P}=\frac{1}{2} \Omega_{2} x_{3} H\left(-x_{1}\right) \delta\left(x_{2}\right), \\
& e_{22}^{P}=\left(\Omega_{3} x_{1}-\Omega_{1} x_{3}\right) H\left(-x_{1}\right) \delta\left(x_{2}\right), \\
& e_{23}^{P}=-\frac{1}{2} \Omega_{2} x_{1} H\left(-x_{1}\right) \delta\left(x_{2}\right),
\end{aligned}
$$

from (4.3), and by (4.6) we find that the components of the plastic bend-twist are

$$
\begin{aligned}
& \kappa_{11}^{P}=-\frac{1}{2} \Omega_{2} H\left(-x_{1}\right) \delta\left(x_{2}\right), \\
& \kappa_{21}^{P}=-\frac{1}{2} \Omega_{2} x_{1} H\left(-x_{1}\right) \delta^{\prime}\left(x_{2}\right)+\Omega_{1} H\left(-x_{1}\right) \delta\left(x_{2}\right), \\
& \kappa_{31}^{P}=0, \\
& \kappa_{12}^{P}=0, \\
& \kappa_{22}^{P}=\Omega_{2} H\left(-x_{1}\right) \delta\left(x_{2}\right), \\
& \kappa_{32}^{P}=0 . \\
& \kappa_{13}^{P}=\frac{1}{2} \Omega_{2} x_{3} \delta\left(x_{1}\right) \delta\left(x_{2}\right), \\
& \kappa_{23}^{P}=-\frac{1}{2} \Omega_{2} x_{3} H\left(-x_{1}\right) \delta^{\prime}\left(x_{2}\right)+\Omega_{3} H\left(-x_{1}\right) \delta\left(x_{2}\right), \\
& \kappa_{33}^{P}=-\frac{1}{2} \Omega_{2} H\left(-x_{1}\right) \delta\left(x_{2}\right),
\end{aligned}
$$

from (4.3-4), where we have used (A6). The defect loop densities (4.3-4) and the basic plastic fields (4.7-8) are singular on the plane $S$, figure 1 . This is the plane on which a discrete amount of plastic displacement has taken place to create the disclination line along the $x_{3}$ axis.

The general expressions for the dislocation and disclination densities are given by, (II5.19-20),

$$
\begin{aligned}
& \alpha_{p l}(\mathbf{r})=\delta_{p}(L)\left\{b_{l}+\epsilon_{l q r} \Omega_{q}\left(x_{r}-x_{r}^{0}\right)\right\}, \\
& \theta_{p q}(\mathbf{r})=\delta_{p}(L) \Omega_{q},
\end{aligned}
$$

where $\delta_{p}(L)$ is the Dirac delta function on the curve $L$, defined by (A11). For our special case, with $b_{l}=x_{r}^{0}=0$, the only nonvanishing components of the defect densities are

$$
\alpha_{3 l}=\epsilon_{l q r} \Omega_{q} x_{r} \delta\left(x_{1}\right) \delta\left(x_{2}\right),
$$




$$
\theta_{3 q}=\Omega_{q} \delta\left(x_{1}\right) \delta\left(x_{2}\right)
$$

by (A15). Again, for convenience, we write these results out in full

$$
\begin{aligned}
& \alpha_{31}=\Omega_{2} x_{3} \delta\left(x_{1}\right) \delta\left(x_{2}\right), \\
& \alpha_{32}=-\Omega_{1} x_{3} \delta\left(x_{1}\right) \delta\left(x_{1}\right), \\
& \alpha_{33}=0, \\
& \theta_{31}=\Omega_{1} \delta\left(x_{1}\right) \delta\left(x_{2}\right), \\
& \theta_{32}=\Omega_{2} \delta\left(x_{1}\right) \delta\left(x_{2}\right), \\
& \theta_{33}=\Omega_{3} \delta\left(x_{1}\right) \delta\left(x_{2}\right),
\end{aligned}
$$

where we have used (A8). The defect densities are concentrated on the $x_{3}$ axis. We illustrate the three different types of disclinations for $q=1,2,3$ in figures 6-8. Figures 6-7 represent twist disclinations because the Frank vectors $\Omega_{1}$ and $\Omega_{2}$ are normal to the disclination line, whereas figure 8 represents a wedge disclination because the Frank vector $\Omega_{3}$ is parallel to the disclination line. From (4.12) we see that the twist disclinations correspond to the off-diagonal components of the disclination density, whereas the wedge disclination corresponds to the diagonal component (see table II1). Note also that the discrete straight twist disclination contains a certain amount of dislocation density, given by $(4.11 \mathrm{a}-\mathrm{b})$. The significance of these expressions can be interpreted as follows. Consider a slice of material parallel to the $x_{1} x_{2}$ plane at the point $x_{3}$. Locally near the

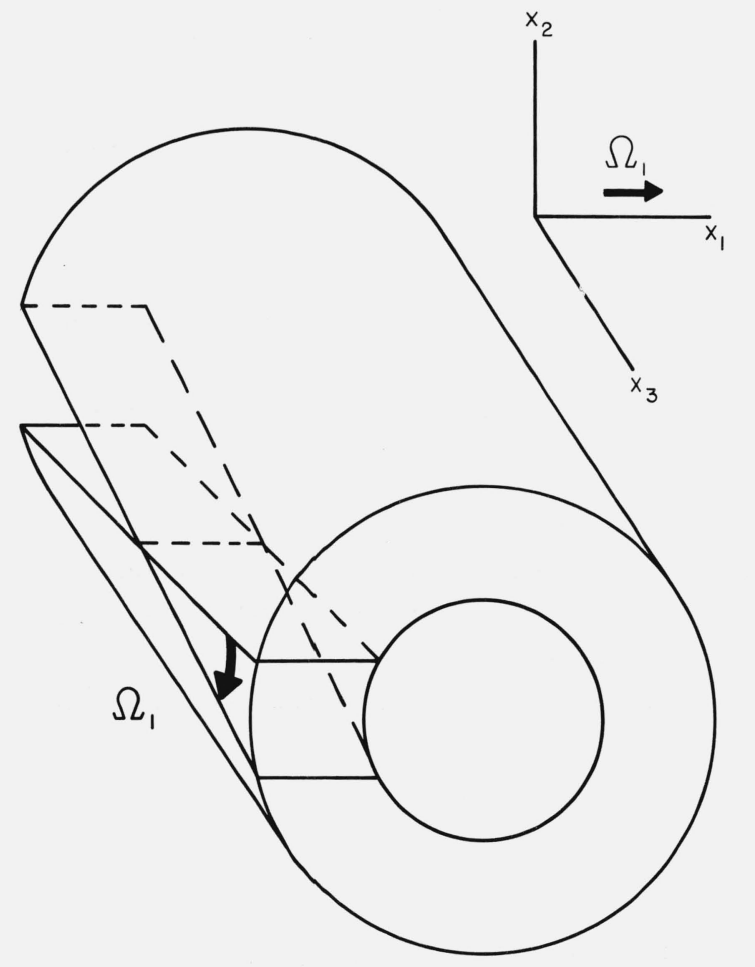

FIGURE 6. Twist disclination with Frank vector $\Omega_{1}$.

It is described by the defect densities $\alpha_{32}=-\Omega_{1} x_{3} \delta\left(x_{1}\right) \delta\left(x_{2}\right)$ and $\theta_{31}=$ $\Omega_{1} \delta\left(x_{1}\right) \delta\left(x_{2}\right)$.

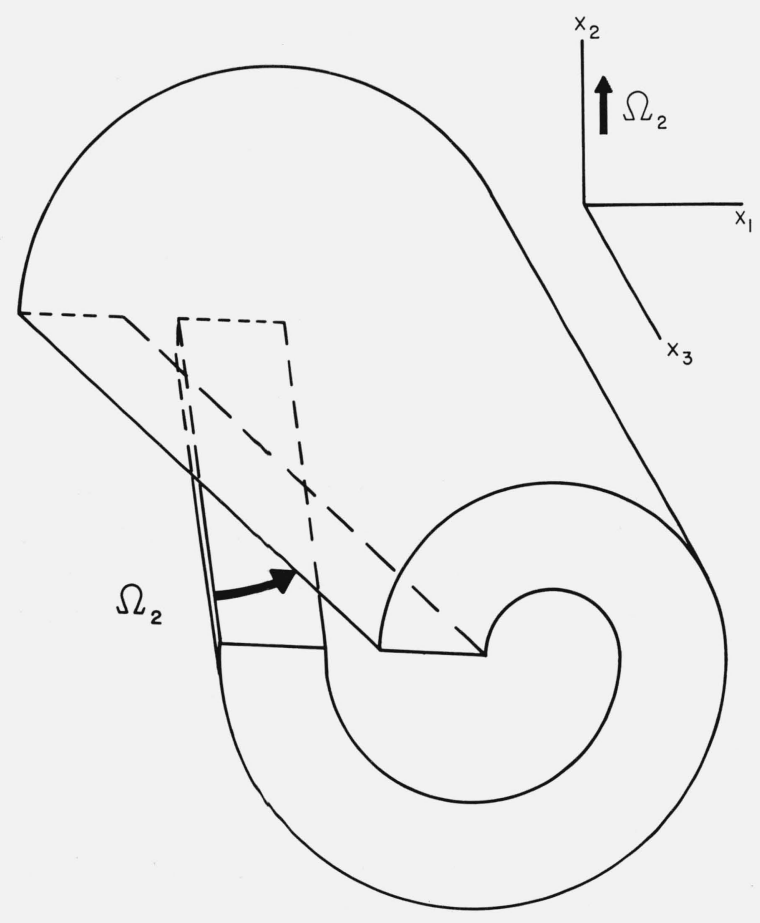

FiguRE 7. Twist disclination with Frank vector $\Omega_{2}$.

It is described by the defect densities $\alpha_{31}=\Omega_{2} x_{3} \delta\left(x_{1}\right) \delta\left(x_{2}\right)$ and $\theta_{32}=\Omega_{2} \delta\left(x_{1}\right) \delta\left(x_{2}\right)$. 


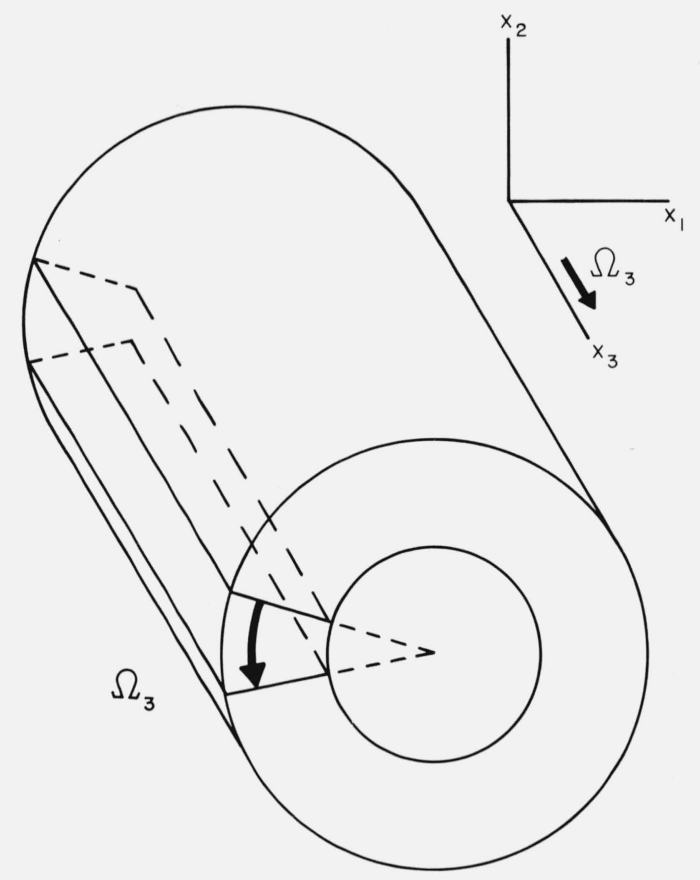

FIGURE 8. Wedge disclination with Frank vector $\Omega_{3}$.

It is described by the disclination density $\theta_{33}=\Omega_{3} \delta\left(x_{1}\right) \delta\left(x_{2}\right)$.

$x_{3}$ axis the displacement jump of the disclination cannot be distinguished from that of a dislocation, even though there is a rotation jump. In figure 6 the deformation and the displacement jump near the point $x_{3}$ is similar to that of the dislocation in figure 3 with the density given by (4.11b). Similarly in figure 7 the deformation near the disclination line is similar to that of the dislocation in figure 2 with the density given by (4.11a). From this point of view it is therefore quite reasonable to regard the discrete disclination line as consisting of a mixture of dislocation and disclination density. This is a particular illustration of the general assertation we have already made in II. Note that the dislocation density implies a dislocation line with changing Burgers vector.

By contrast Anthony[10] does not regard (4.11) as a dislocation density, but as an integral part of the disclination density. The advantage of this approach is that the disclination is unambiguously defined, whether it is described in terms of continuous distribution of defects, or a discrete line. However, this approach becomes cumbersome when we try to describe a terminating dislocation as in section 12. Anthony has also treated several special cases of straight disclinations. For the wedge disclination his equations (27-28) and (30) correspond to our equations (4.12c) and (4.11c). We shall compare his results for the twist disclination with ours in section 6 .

For consistency it is straightforward to verify that the basic plastic fields (4.7-8) and the defect densities (4.11-12) satisfy the general definitions, (I6.3, I6.1), (II4.1-2),

$$
\begin{aligned}
& \alpha_{p l} \equiv-\epsilon_{p m k}\left(e_{k l, m}^{P}+\epsilon_{k l q} \kappa_{m q}^{P}\right), \\
& \theta_{p q} \equiv-\epsilon_{p m k} \kappa_{k q, m^{\prime}}^{P}
\end{aligned}
$$

by (A6) and (A8). Furthermore, it is easy to verify the general relations (II6.27-28) between the loop densities (4.3-4) and the defect densities (4.11-12)

$$
\alpha_{p l}=-\epsilon_{p m k}\left(\beta_{k l, m}^{*}+\epsilon_{k l q} \varphi_{m q}^{*}\right),
$$




$$
\boldsymbol{\theta}_{p q}=-\boldsymbol{\epsilon}_{p m k} \boldsymbol{\varphi}_{k q, m}^{*}
$$

also by (A6) and (A8). We also see that the defect densities (4.11-12) satisfy the general continuity conditions, (I6.8, I6.2), (II5.27-28),

$$
\begin{gathered}
\alpha_{p l, p}+\epsilon_{l p q} \theta_{p q}=0, \\
\theta_{p q, p}=0 .
\end{gathered}
$$

We next wish to calculate the total Burgers vector which is in general defined in terms of the basic plastic fields by, (II4.5),

$$
B_{l}=-\oint_{\lambda}\left(e_{k l}^{P}-\epsilon_{l q r} \kappa_{k q}^{P} x_{r}\right) d L_{k},
$$

where $\lambda$ is the Burgers circuit, figure 1. For our special case we find from (4.7-8) by a somewhat tedious calculation that

$$
B_{l}=0 \text {, }
$$

where we have used (A8-9). The general relation for a discrete defect line is, (II5.17),

$$
B_{l}=b_{l}-\epsilon_{l q r} \Omega_{q} x_{r}^{0}
$$

and with our special assumption that $b_{l}=x_{r}^{0}=0$ this reduces to (4.20). The total Burgers vector $B_{l}$ measures the displacement jump at the origin, which vanishes in figures 6-8. The total Burgers vector can in general also be expressed in terms of the defect loop densities, (II6.31), or the defect densities, (II4.7),

$$
\begin{aligned}
B_{l} & =-\oint_{\lambda}\left(\beta_{k l}^{*}-\epsilon_{l q r} \varphi_{k q}^{*} x_{r}\right) d L_{k} \\
& =\int_{\sigma}\left(\alpha_{p l}-\epsilon_{l q r} \theta_{p q} x_{r}\right) d S_{p},
\end{aligned}
$$

where $\sigma$ is the Burgers surface, figure 1. From (4.3-4) and (4.11-12) it easily follows that these equations lead to the same result as (4.20). Now we take for the Burgers circuit $\lambda$ a circle of radius $a$ in the $x_{1} x_{2}$ plane centered at the origin (fig. 1). Then we have by (4.8) and (A19-21)

$$
-\oint_{\lambda} \kappa_{k q}^{P} d L_{k}=\Omega_{q}
$$

in agreement with (II5.18), confirming that $\Omega_{q}$ is the Frank vector. We also have by (4.4) and (A23)

$$
-\oint_{\lambda} \varphi_{k q}^{*} d L_{k}=-\oint_{\lambda} \Omega_{q} H\left(-x_{1}\right) \delta\left(x_{2}\right) d L_{2}=\Omega_{q}
$$

in agreement with (II6.32), and by (4.12) and (A22)

$$
\int_{\sigma} \theta_{p q} d S_{p}=\int_{\sigma} \Omega_{q} \delta\left(x_{1}\right) \delta\left(x_{2}\right) d S_{3}=\Omega_{q}
$$

in agreement with (II5.30). The jump in displacement across the surface $S$ is in general given by, 
(II5.1, II5.5),

$$
\begin{aligned}
{\left[u_{l}(\mathbf{r})\right] } & =B_{l}+\epsilon_{l q r} \Omega_{q} x_{r} \\
& =b_{l}+\epsilon_{l q r} \Omega_{q}\left(x_{r}-x_{r}^{0}\right) .
\end{aligned}
$$

In our special case with $b_{l}=x_{r}^{0}=0$, or by $(4.20)$, this reduces to

$$
\left[u_{l}(\mathbf{r})\right]=\epsilon_{l q r} \Omega_{q} x_{r} .
$$

This jump agrees with those depicted in figures 6-8. So, in this section we have found the special forms of the defect loop densities (4.3-4), the basic plastic fields (4.7-8), and the defect densities (4.11-12), for a straight disclination running along the $x_{3}$ axis, and these equations are in complete agreement with all the general results for geometry from II.

We conclude this section with expressions for the incompatability tensor. It is in general given by, (I6.6), (II4.12),

$$
\eta_{p q}=-\left(\epsilon_{q n l} \alpha_{p l, n}+\theta_{p q}\right)_{(p q)}
$$

For our special case we find the only nonvanishing components from (4.11-12)

$$
\begin{aligned}
& \eta_{13}=-\Omega_{1} \delta\left(x_{1}\right) \delta\left(x_{2}\right), \\
& \eta_{23}=-\Omega_{2} \delta\left(x_{1}\right) \delta\left(x_{2}\right) \\
& \eta_{33}=\Omega_{1} x_{3} \delta^{\prime}\left(x_{1}\right) \delta\left(x_{2}\right)+\Omega_{2} x_{3} \delta\left(x_{1}\right) \delta^{\prime}\left(x_{2}\right)-\Omega_{3} \delta\left(x_{1}\right) \delta\left(x_{2}\right),
\end{aligned}
$$

by (A9). We note that these results satisfy the continuity condition, (I4.2), (II4.13),

$$
\eta_{p q, p}=0 .
$$

The results in this section apply to an infinitely extended body, whether isotropic or anisotropic.

\section{Isotropic Statics of the Straight Disclination}

In this section we find the elastostatic fields for a straight disclination in an infinitely extended isotropic body. If the Volterra expressions (2.1) are properly modified to maintain equilibrium everywhere, then the displacement is given by (appendix $\mathrm{C}$ )

$$
\begin{aligned}
& u_{1}^{T}=-\frac{\Omega_{1} x_{3}}{4 \pi(1-\nu)}\left[(1-2 \nu) \ln \rho+\frac{x_{2}^{2}}{\rho^{2}}\right]+\Omega_{2} x_{3}\left[\frac{\varphi}{2 \pi}+\frac{x_{1} x_{2}}{4 \pi(1-\nu) \rho^{2}}\right] \\
& -\Omega_{3}\left[\frac{x_{2} \varphi}{2 \pi}-\frac{1-2 \nu}{4 \pi(1-\nu)} x_{1}(\ln \rho-1)\right] \text {, } \\
& u_{2}^{T}=-\Omega_{1} x_{3}\left[\frac{\varphi}{2 \pi}-\frac{x_{1} x_{2}}{4 \pi(1-\nu) \rho^{2}}\right]-\frac{\Omega_{2} x_{3}}{4 \pi(1-\nu)}\left[(1-2 \nu) \ln \rho+\frac{x_{1}^{2}}{\rho^{2}}\right] \\
& +\Omega_{3}\left[\frac{x_{1} \varphi}{2 \pi}+\frac{1-2 \nu}{4 \pi(1-\nu)} x_{2}(\ln \rho-1)\right], \\
& u_{3}^{T}=\Omega_{1}\left[\frac{x_{2} \varphi}{2 \pi}-\frac{1-2 \nu}{4 \pi(1-\nu)} x_{1}(\ln \rho-1)\right]-\Omega_{2}\left[\frac{x_{1} \varphi}{2 \pi}+\frac{1-2 \nu}{4 \pi(1-\nu)} x_{1}(\ln \rho-1)\right],
\end{aligned}
$$


where $\nu$ is Poisson's ratio. We have used a Cartesian coordinate system $x_{1}, x_{2}, x_{3}$, as shown in figure 1, and the cylindrical coordinates $\rho$ and $\phi$, as defined in appendix B and shown in figure 2 . This is the displacement field of a straight disclination line running along the $x_{3}$ axis, with Frank vector $\Omega_{q}$. By restricting the angle $\phi$ to the range $(-\pi, \pi)$ we shall find that the semi-infinite plane $S$ becomes the natural plastic displacement surface of section 4 . The asymptotic dependence of the displacement (5.1) goes as $\rho \ln \rho$, in agreement with table II3.

The total distortion is in general defined in terms of the displacement by, (I2.1), (II3.9),

$$
\beta_{k l}^{T} \equiv u_{l, k}^{T} .
$$

In performing these differentiations in the displacement (5.1) we shall regard three-dimensional space to consist of the product of the two-dimensional $x_{1} x_{2}$ plane and the independent onedimensional $x_{3}$ line. For $x_{1}$ and $x_{2}$ we use the results for generalized derivatives of appendix B. Since $x_{3}$ appears only linearly in (5.1) the classical and generalized derivatives with respect to $x_{3}$ coincide. So we find for the total distortion, using (B3-7),

$$
\begin{array}{r}
\beta_{11}^{T}=-\frac{\Omega_{1} x_{3}}{4 \pi(1-\nu)}\left[(1-2 \nu) \frac{x_{1}}{\rho^{2}}-2 \frac{x_{1} x_{2}^{2}}{\rho^{4}}\right]-\frac{\Omega_{2} x_{3}}{4 \pi(1-\nu)}\left[(1-2 \nu) \frac{x_{2}}{\rho^{2}}+2 \frac{x_{1}^{2} x_{2}}{\rho^{4}}\right] \\
+\frac{\Omega_{3}}{4 \pi(1-\nu)}\left[(1-2 \nu) \ln \rho+\frac{x_{2}^{2}}{\rho^{2}}\right], \\
\begin{aligned}
\beta_{21}^{T}=-\frac{\Omega_{1} x_{3}}{4 \pi(1-\nu)}\left[(1-2 \nu) \frac{x_{2}}{\rho^{2}}+2 \frac{x_{1}^{2} x_{2}}{\rho^{4}}\right]+ & \frac{\Omega_{2} x_{3}}{4 \pi(1-\nu)}\left[(3-2 \nu) \frac{x_{1}}{\rho^{2}}-2 \frac{x_{1} x_{2}^{2}}{\rho^{4}}\right] \\
& +\Omega_{2} x_{3} H\left(-x_{1}\right) \delta\left(x_{2}\right)-\Omega_{3}\left[\frac{\varphi}{2 \pi}+\frac{x_{1} x_{2}}{4 \pi(1-\nu) \rho^{2}}\right],
\end{aligned}
\end{array}
$$

$\beta_{31}^{T}=-\frac{\Omega_{1}}{4 \pi(1-\nu)}\left[(1-2 \nu) \ln \rho+\frac{x_{2}^{2}}{\rho^{2}}\right]+\Omega_{2}\left[\frac{\varphi}{2 \pi}+\frac{x_{1} x_{2}}{4 \pi(1-\nu) \rho^{2}}\right]$,

$\beta_{12}^{T}=\frac{\Omega_{1} x_{3}}{4 \pi(1-\nu)}\left[(3-2 \nu) \frac{x_{2}}{\rho^{2}}-2 \frac{x_{1}^{2} x_{2}}{\rho^{4}}\right]$

$$
-\frac{\Omega_{2} x_{3}}{4 \pi(1-\nu)}\left[(1-2 \nu) \frac{x_{1}}{\rho^{2}}+2 \frac{x_{1} x_{2}^{2}}{\rho^{4}}\right]+\Omega_{3}\left[\frac{\varphi}{2 \pi}-\frac{x_{1} x_{2}}{4 \pi(1-\nu) \rho^{2}}\right],
$$

$\beta_{22}^{T}=-\frac{\Omega_{1} x_{3}}{4 \pi(1-\nu)}\left[(1-2 \nu) \frac{x_{1}}{\rho^{2}}+2 \frac{x_{1} x_{2}^{2}}{\rho^{4}}\right]-\Omega_{1} x_{3} H\left(-x_{1}\right) \delta\left(x_{2}\right)$

$-\frac{\Omega_{2} x_{3}}{4 \pi(1-\nu)}\left[(1-2 \nu) \frac{x_{2}}{\rho^{2}}-2 \frac{x_{1}^{2} x_{2}}{\rho^{4}}\right]+\frac{\Omega_{3}}{4 \pi(1-\nu)}\left[(1-2 \nu) \ln \rho+\frac{x_{1}^{2}}{\rho^{2}}\right]+\Omega_{3} x_{1} H\left(-x_{1}\right) \delta\left(x_{2}\right)$,

$\beta_{32}^{T}=-\Omega_{1}\left[\frac{\varphi}{2 \pi}-\frac{x_{1} x_{2}}{4 \pi(1-\nu) \rho^{2}}\right]-\frac{\Omega_{2}}{4 \pi(1-\nu)}\left[(1-2 \nu) \ln \rho+\frac{x_{1}^{2}}{\rho^{2}}\right]$,

$\beta_{13}^{T}=-\frac{\Omega_{1}}{4 \pi(1-\nu)}\left[(1-2 \nu) \ln \rho+\frac{x_{2}^{2}}{\rho^{2}}\right]-\Omega_{2}\left[\frac{\varphi}{2 \pi}-\frac{x_{1} x_{2}}{4 \pi(1-\nu) \rho^{2}}\right]$,

$\beta_{23}^{T}=\Omega_{1}\left[\frac{\varphi}{2 \pi}+\frac{x_{1} x_{2}}{4 \pi(1-\nu) \rho^{2}}\right]-\frac{\Omega_{2}}{4 \pi(1-\nu)}\left[(1-2 \nu) \ln \rho+\frac{x_{1}^{2}}{\rho^{2}}\right]-\Omega_{2} x_{1} H\left(-x_{1}\right) \delta\left(x_{2}\right)$,

$\beta_{33}^{T}=0$. 
Note that the total distortion contains terms which are singular on $S$ and which correspond exactly to the dislocation loop density $\beta_{k l}^{*}$ given by (4.3). This result is in agreement with the general equation (II5.34). Therefore we may be tempted to regard these terms as the plastic distortion $\beta_{k l}^{P}$ and consequently find the elastic distortion from (3.13). This is the point of view taken by Mura [9]. We shall investigate the consequences of this approach in section 8. Strictly speaking we are at liberty to split the total distortion in any way we wish into an elastic and a plastic part. However, the present theory then implies that there are no disclinations. We note, though, that the total distortion (5.3) still contains the angle $\phi$, in contrast to the dislocation case. Since $\phi$ is discontinuous at the surface $S$, it is not possible to split it into a part that is continuous everywhere except at the $x_{3}$ axis, and a part that is localized only on the surface $S$. Therefore the total distortion cannot be split into an elastic part that should be continuous everywhere except at the $x_{3}$ axis and a plastic part that is localized on the surface $S$ of plastic displacement. We shall find, however, that the basic total fields no longer contain the angle $\phi$ and so it will be easy to find an intuitively reasonable way to split them into an elastic and plastic part.

We first consider the total strain which is the symmetric part of the total distortion and consists of an elastic and plastic part, (II4.14),

$$
e_{k l}^{T} \equiv \beta_{(k l)}^{T}=e_{k l}+e_{k l}^{P} .
$$

If we use (5.3) in this expression we shall find that the terms which are singular on $S$ correspond exactly to the plastic strain $e_{k l}^{P}$ given by (4.7). So we find for the elastic strain of the straight disclination

$$
\begin{aligned}
& e_{11}=-\frac{\Omega_{1} x_{3}}{4 \pi(1-\nu)}\left[(1-2 \nu) \frac{x_{1}}{\rho^{2}}-2 \frac{x_{1} x_{2}^{2}}{\rho^{4}}\right]-\frac{\Omega_{2} x_{3}}{4 \pi(1-\nu)}\left[(1-2 \nu) \frac{x_{2}}{\rho^{2}}+2 \frac{x_{1}^{2} x_{2}}{\rho^{4}}\right] \\
& +\frac{\Omega_{3}}{4 \pi(1-\nu)}\left[(1-2 \nu) \ln \rho+\frac{x_{2}^{2}}{\rho^{2}}\right], \\
& e_{22}=-\frac{\Omega_{1} x_{3}}{4 \pi(1-\nu)}\left[(1-2 \nu) \frac{x_{1}}{\rho^{2}}+2 \frac{x_{1} x_{2}^{2}}{\rho^{4}}\right]-\frac{\Omega_{2} x_{3}}{4 \pi(1-\nu)}\left[(1-2 \nu) \frac{x_{2}}{\rho^{2}}-2 \frac{x_{1}^{2} x_{2}}{\rho^{4}}\right] \\
& +\frac{\Omega_{3}}{4 \pi(1-\nu)}\left[(1-2 \nu) \ln \rho+\frac{x_{1}^{2}}{\rho^{2}}\right] \text {, }
\end{aligned}
$$

$e_{33}=0$,

$e_{12}=\frac{\Omega_{1} x_{3}}{4 \pi(1-\nu)}\left[\frac{x_{2}}{\rho^{2}}-2 \frac{x_{1}^{2} x_{2}}{\rho^{4}}\right]+\frac{\Omega_{2} x_{3}}{4 \pi(1-\nu)}\left[\frac{x_{1}}{\rho^{2}}-2 \frac{x_{1} x_{2}^{2}}{\rho^{4}}\right]-\frac{\Omega_{3} x_{1} x_{2}}{4 \pi(1-\nu) \rho^{2}}$,

$e_{23}=\frac{\Omega_{1} x_{1} x_{2}}{4 \pi(1-\nu) \rho^{2}}-\frac{\Omega_{2}}{4 \pi(1-\nu)}\left[(1-2 \nu) \ln \rho+\frac{x_{1}^{2}}{\rho^{2}}\right]$,

$e_{31}=-\frac{\Omega_{1}}{4 \pi(1-\nu)}\left[(1-2 \nu) \ln \rho+\frac{x_{2}^{2}}{\rho^{2}}\right]+\frac{\Omega_{2} x_{1} x_{2}}{4 \pi(1-\nu) \rho^{2}}$,

The dilatation $e=e_{k k}$ is

$$
e=-\frac{(1-2 \nu) x_{3}}{2 \pi(1-\nu) \rho^{2}}\left(\Omega_{1} x_{1}+\Omega_{2} x_{2}\right)+\frac{\Omega_{3}}{4 \pi(1-\nu)}[2(1-2 \nu) \ln \rho-1] .
$$

The elastic strain consists of functions that are continuous everywhere except at the $x_{3}$ axis, which is reasonable to expect for an elastic field. These expressions also show a logarithmic dependence 
for the elastic strain at large distances from the disclination line, in agreement with table II3.

To find the total bend-twist we first find the total rotation which is in general given by, (III5.11),

$$
\omega_{q}^{T}=\frac{1}{2} \epsilon_{k l q} \beta_{k l}^{T},
$$

or, from (5.3), for our special case by

$$
\begin{aligned}
& \omega_{1}^{T}=\frac{\Omega_{1} \varphi}{2 \pi}-\frac{1}{2} \Omega_{2} x_{1} H\left(-x_{1}\right) \delta\left(x_{2}\right), \\
& \omega_{2}^{T}=\frac{\Omega_{2} \varphi}{2 \pi}, \\
& \omega_{3}^{T}=\frac{\Omega_{1} x_{2} x_{3}}{2 \pi \rho^{2}}-\frac{\Omega_{2} x_{1} x_{3}}{2 \pi \rho^{2}}-\frac{1}{2} \Omega_{2} x_{3} H\left(-x_{1}\right) \delta\left(x_{2}\right)+\frac{\Omega_{3} \varphi}{2 \pi} .
\end{aligned}
$$

These expressions still contain the angle $\phi$, which prevents us from splitting the total rotation into an elastic and plastic part in a reasonable way. The total bend-twist is in general defined in terms of the total rotation and can be split into an elastic and plastic part, (II4.15),

$$
\kappa_{k q}^{T} \equiv \omega_{q, k}^{T}=\kappa_{k q}+\kappa_{k q}^{P}
$$

In carrying out this differentiation on the functions in (5.7) we shall find that the resulting terms singular on $S$ correspond exactly to the plastic bend-twist $\kappa_{k q}^{P}$ given by (4.8). So we find for the elastic bend-twist, using also (A6), (B3-4), and (B8),

$$
\begin{aligned}
& \kappa_{11}=-\frac{\Omega_{1} x_{2}}{2 \pi \rho^{2}}, \\
& \kappa_{21}=\frac{\Omega_{1} x_{1}}{2 \pi \rho^{2}}, \\
& \kappa_{31}=0, \\
& \kappa_{12}=-\frac{\Omega_{2} x_{2}}{2 \pi \rho^{2}}, \\
& \kappa_{22}=\frac{\Omega_{2} x_{1}}{2 \pi \rho^{2}}, \\
& \kappa_{32}=0, \\
& \kappa_{13}=-\frac{\Omega_{1} x_{1} x_{2} x_{3}}{\pi \rho^{4}}-\frac{\Omega_{2} x_{3}}{2 \pi}\left[\frac{1}{\rho^{2}}-2 \frac{x_{1}^{2}}{\rho^{4}}+\pi \delta(\boldsymbol{\rho})\right]-\frac{\Omega_{3} x_{2}}{2 \pi \rho^{2}}, \\
& \kappa_{23}=\frac{\Omega_{1} x_{3}}{2 \pi}\left[\frac{1}{\rho^{2}}-2 \frac{x_{2}^{2}}{\rho^{4}}+\pi \delta(\boldsymbol{\rho})\right]+\frac{\Omega_{2} x_{1} x_{2} x_{3}}{\pi \rho^{4}}+\frac{\Omega_{3} x_{1}}{2 \pi \rho^{2}} \\
& \kappa_{33}=\frac{\Omega_{1} x_{2}}{2 \pi \rho^{2}}-\frac{\Omega_{2} x_{1}}{2 \pi \rho^{2}} \cdot
\end{aligned}
$$


The elastic bend-twist, like the elastic strain, no longer contains the angle $\phi$ and is continuous everywhere except at the $x_{3}$ axis. Alternatively, if we had no a priori knowledge of the basic plastic fields (4.7-8), we could assign to them the terms in the basic total fields that are singular on $S$, because these surface singularities intuitively show where the plastic displacement has taken place. In this manner we would then also have been able to obtain the basic elastic fields (5.5) and (5.9), starting only with the expressions for the displacement (5.1), as discussed at the end of section 2 .

To check the consistency of our results, it can be verified that the basic elastic fields (5.5) and (5.9) and the defect densities (4.11-12) satisfy the field equations, (I6.3, I6.1), (II4.17-18),

$$
\begin{gathered}
\boldsymbol{\epsilon}_{p m k}\left(e_{k l, m}+\boldsymbol{\epsilon}_{k l q} \kappa_{m q}\right)=\alpha_{p l}, \\
\boldsymbol{\epsilon}_{p m k} \kappa_{k q, m}=\theta_{p q},
\end{gathered}
$$

by (B8-12). These results then confirm that (5.5) and (5.9) represent the basic elastic fields of a discrete disclination line running along the $x_{3}$ axis. Furthermore it can be verified that the elastic strain (5.5) and the incompatibility tensor (4.31) satisfy the field equation, (II4.22),

$$
-\epsilon_{p m k} \epsilon_{q n l} e_{k l, m n}=\eta_{p q},
$$

by $(\mathrm{B} 8-12)$.

Next we want to show that equilibrium is maintained everywhere. We first find the stress from Hookes' law (3.18) and the elastic strain (5.5)

$$
\begin{aligned}
& \sigma_{11}=-\frac{G \Omega_{1} x_{3}}{2 \pi(1-\nu)}\left[\frac{x_{1}}{\rho^{2}}-2 \frac{x_{1} x_{2}^{2}}{\rho^{4}}\right]-\frac{G \Omega_{2} x_{3}}{2 \pi(1-\nu)}\left[\frac{x_{2}}{\rho^{2}}+2 \frac{x_{1}^{2} x_{2}}{\rho^{4}}\right] \\
& \quad+\frac{G \Omega_{3}}{2 \pi(1-\nu)}\left[\ln \rho+\frac{x_{2}^{2}}{\rho^{2}}+\frac{\nu}{1-2 \nu}\right], \\
& \sigma_{22}=-\frac{G \Omega_{2} x_{3}}{2 \pi(1-\nu)}\left[\frac{x_{1}}{\rho^{2}}+2 \frac{x_{1} x_{2}^{2}}{\rho^{4}}\right]-\frac{G \Omega_{2} x_{3}}{2 \pi(1-\nu)}\left[\frac{x_{2}}{\rho^{2}}-2 \frac{x_{1}^{2} x_{2}}{\rho^{4}}\right] \\
& \quad+\frac{G \Omega_{3}}{2 \pi(1-\nu)}\left[\ln \rho+\frac{x_{1}^{2}}{\rho^{2}}+\frac{\nu}{1-2 \nu}\right], \\
& \sigma_{33}=-\frac{G \nu x_{3}}{\pi(1-\nu) \rho^{2}}\left(\Omega_{1} x_{1}+\Omega_{2} x_{2}\right)+\frac{G \Omega_{3}}{2 \pi(1-\nu)}\left[2 \nu \ln \rho+\frac{\nu}{1-2 \nu}\right], \\
& \sigma_{12}=\frac{G \Omega_{1} x_{3}}{2 \pi(1-\nu)}\left[\frac{x_{2}}{\rho^{2}}-2 \frac{x_{1}^{2} x_{2}}{\rho^{4}}\right]+\frac{G \Omega_{2} x_{3}}{2 \pi(1-\nu)}\left[\frac{x_{1}}{\rho^{2}}-2 \frac{x_{1} x_{2}^{2}}{\rho^{4}}\right]-\frac{G \Omega_{3} x_{1} x_{2}}{2 \pi(1-\nu) \rho^{2}}, \\
& \sigma_{23}=\frac{G \Omega_{1} x_{1} x_{2}}{2 \pi(1-\nu) \rho^{2}}-\frac{G \Omega_{2}}{2 \pi(1-\nu)}\left[(1-2 \nu) \ln \rho+\frac{x_{1}^{2}}{\rho^{2}}\right], \\
& \sigma_{31}=-\frac{G \Omega_{1}}{2 \pi(1-\nu)}\left[(1-2 \nu) \ln \rho+\frac{x_{2}^{2}}{\rho^{2}}\right]+\frac{G \Omega_{2} x_{1} x_{2}}{2 \pi(1-\nu) \rho^{2}} \cdot
\end{aligned}
$$

If we substitute these results into the equations of equilibrium (3.20) we find by (B5-10) that

$$
f_{l}=0 \text {, }
$$

everywhere, so that the above fields maintain equilibrium without body forces, including at the $x_{3}$ axis. The displacement (5.1) contains the characteristic Volterra terms (2.1) for a disclination. 
Therefore, as discussed in section 2 , we may conclude that (5.1) correctly represents the displacement of a straight disclination line.

In this section we have presented the static fields, i.e., the displacement, total distortion, basic elastic fields (strain and bend-twist), and stress, of a straight disclination line in an infinite isotropic body.

\section{Movement of the Disclination Axis}

If we do not restrict the disclination axis from passing through the origin as in sections 4-5 we obtain more general results. These can be found by removing the restriction $x_{r}^{0}=0$, which was used in those sections to find the explicit formulas from the general expressions of II. In this section we shall discuss a few selected expressions which are of particular interest. They will show that the effect of moving the disclination axis is equivalent to adding a dislocation, as was already pointed out in II.

\subsection{Geometry}

The general expression for the dislocation density of a discrete defect line is given by (4.9). We shall now consider the special case of a discrete disclination line lying along the $x_{3}$ axis, but whose rotation axis passes through the general point $x_{r}^{0}$. Then the only nonvanishing components of the dislocation density become by (A15), setting $b_{l}=0$,

$$
\alpha_{3 l}=\epsilon_{l q r} \Omega_{q}\left(x_{r}-x_{r}^{0}\right) \delta\left(x_{1}\right) \delta\left(x_{2}\right),
$$

instead of (4.11). On the other hand, the disclination density of our discrete disclination line with its axis shifted from the origin remains the same as (4.12). When written out in full we have for (6.1)

$$
\begin{aligned}
& \alpha_{31}=\left[\Omega_{2}\left(x_{3}-x_{3}^{0}\right)+\Omega_{3} x_{2}^{0}\right] \delta\left(x_{1}\right) \delta\left(x_{2}\right), \\
& \alpha_{32}=\left[-\Omega_{3} x_{1}^{0}-\Omega_{1}\left(x_{3}-x_{3}^{0}\right)\right] \delta\left(x_{1}\right) \delta\left(x_{2}\right), \\
& \alpha_{33}=\left[-\Omega_{1} x_{2}^{0}+\Omega_{2} x_{1}^{0}\right] \delta\left(x_{1}\right) \delta\left(x_{2}\right),
\end{aligned}
$$

where we had used (A8). These results of course reduce to (4.11) when $x_{r}^{0}=0$. In the present case we find that the total Burgers vector no longer vanishes,

$$
B_{l}=-\epsilon_{l q r} \Omega_{q} x_{r}^{0},
$$

from (4.21) with $b_{l}=0$. So the displacement jump across the surface $S$ for our special case becomes

$$
\left[u_{l}(\mathbf{r})\right]=\epsilon_{l q r} \Omega_{q}\left(x_{r}-x_{r}^{0}\right),
$$

from (4.27-28), or, when written out in detail

$$
\begin{aligned}
& {\left[u_{1}(\mathbf{r})\right]=\Omega_{2}\left(x_{3}-x_{3}^{0}\right)-\Omega_{3}\left(x_{2}-x_{2}^{0}\right),} \\
& {\left[u_{2}(\mathbf{r})\right]=\Omega_{3}\left(x_{1}-x_{1}^{0}\right)-\Omega_{1}\left(x_{3}-x_{3}^{0}\right),} \\
& {\left[u_{3}(\mathbf{r})\right]=\Omega_{1}\left(x_{2}-x_{2}^{0}\right)-\Omega_{2}\left(x_{1}-x_{1}^{0}\right) .}
\end{aligned}
$$

The total Burgers vector (6.2) is the displacement jump at the origin. Anthony [10] has treated the special case of the twist disclination, $\Omega_{1}$, for the case when the axis passes through the point $x_{2}^{0}=x_{2}(Z), x_{3}^{0}=0$. To find the results for his case we set $\Omega_{2}=\Omega_{3}=0$ in the above equations. The 
only nonvanishing components of (4.12), (6.1), and (6.3) then are

$$
\begin{aligned}
\theta_{31} & =\Omega_{1} \delta\left(x_{1}\right) \delta\left(x_{2}\right), \\
\alpha_{32} & =-\Omega_{1} x_{3} \delta\left(x_{1}\right) \delta\left(x_{2}\right), \\
\alpha_{33} & =-\Omega_{1} x_{2}(Z) \delta\left(x_{1}\right) \delta\left(x_{2}\right), \\
{\left[u_{2}\right] } & =-\Omega_{1} x_{3}\left(P_{0}\right), \\
{\left[u_{3}\right] } & =\Omega_{1}\left(x_{2}\left(P_{0}\right)-x_{2}(Z)\right),
\end{aligned}
$$

where we have set $x_{2}=x_{2}\left(P_{0}\right), x_{3}=x_{3}\left(P_{0}\right)$. These results correspond to Anthony's equations (31), (33), and (34). Hence our results are in complete agreement with Anthony.

If we compare (6.1) with (4.11) we see that the axis has been moved from the origin to the point $x_{r}^{0}$ by adding the dislocation density

$$
\alpha_{3 l}=-\epsilon_{l q r} \Omega_{q} x_{r}^{0} \delta\left(x_{1}\right) \delta\left(x_{2}\right)
$$

to the disclination line of section 4. This is in agreement with (II5.24). From (3.4) this corresponds to adding a dislocation line with Burgers vector

$$
b_{l}=-\epsilon_{l q r} \Omega_{q} x_{r}^{0}
$$

to the disclination line, in agreement with (II5.23). In detail (6.8) is

$$
\begin{aligned}
& b_{1}=-\Omega_{2} x_{3}^{0}+\Omega_{3} x_{2}^{0}, \\
& b_{2}=-\Omega_{3} x_{1}^{0}+\Omega_{1} x_{3}^{0}, \\
& b_{3}=-\Omega_{1} x_{2}^{0}+\Omega_{2} x_{1}^{0} .
\end{aligned}
$$

So the disclination axis is moved from the origin to $x_{r}^{0}$ by simply adding a dislocation with Burgers vector (6.8) to it.

\subsection{Statics}

The fields of a straight discrete disclination along the $x_{3}$ axis whose rotation axis passes through the point $x_{r}^{0}$ can be obtained by adding the fields of the dislocation line in section 3.2 with the Burgers vector given by (6.8) to the fields of the disclination line in section 5 . For example, the displacement is obtained by substituting (6.8) into (3.12) and adding it to (5.1)

$$
\begin{aligned}
& u_{1}^{T}=-\frac{\Omega_{1}\left(x_{3}-x_{3}^{0}\right)}{4 \pi(1-\nu)}\left[(1-2 \nu) \ln \rho+\frac{x_{2}^{2}}{\rho^{2}}\right]+\Omega_{2}\left(x_{3}-x_{3}^{0}\right)\left[\frac{\varphi}{2 \pi}+\frac{x_{1} x_{2}}{4 \pi(1-\nu) \rho^{2}}\right] \\
& +\frac{\Omega_{3}\left(x_{1}-x_{1}^{0}\right)}{4 \pi(1-\nu)}\left[(1-2 \nu) \ln \rho+\frac{x_{2}^{2}}{\rho^{2}}\right]-\Omega_{3}\left(x_{2}-x_{2}^{0}\right)\left[\frac{\varphi}{2 \pi}+\frac{x_{1} x_{2}}{4 \pi(1-\nu) \rho^{2}}\right]-\Omega_{3} \frac{1-2 \nu}{4 \pi(1-\nu)} x_{1}, \\
& u_{2}^{T}=-\Omega_{1}\left(x_{3}-x_{3}^{0}\right)\left[\frac{\varphi}{2 \pi}-\frac{x_{1} x_{2}}{4 \pi(1-\nu) \rho^{2}}\right]-\frac{\Omega_{2}\left(x_{3}-x_{3}^{0}\right)}{4 \pi(1-\nu)}\left[(1-2 \nu) \ln \rho+\frac{x_{1}^{2}}{\rho^{2}}\right] \\
& +\Omega_{3}\left(x_{1}-x_{1}^{0}\right)\left[\frac{\varphi}{2 \pi}-\frac{x_{1} x_{2}}{4 \pi(1-\nu) \rho^{2}}\right]+\frac{\Omega_{3}\left(x_{2}-x_{2}^{0}\right)}{4 \pi(1-\nu)}\left[(1-2 \nu) \ln \rho+\frac{x_{1}^{2}}{\rho^{2}}\right]-\Omega_{3} \frac{1-2 \nu}{4 \pi(1-\nu)} x_{2},
\end{aligned}
$$


$u_{3}^{T}=\Omega_{1}\left[\frac{\left(x_{2}-x_{2}^{0}\right) \varphi}{2 \pi}-\frac{1-2 \nu}{4 \pi(1-\nu)} x_{1}(\ln \rho-1)\right]-\Omega_{2}\left[\frac{\left(x_{1}-x_{1}^{0}\right) \varphi}{2 \pi}+\frac{1-2 \nu}{4 \pi(1-\nu)} x_{2}(\ln \rho-1)\right]$

This is the displacement of a straight disclination line running along the $x_{3}$ axis, with a rotation axis that passes thru the point $x_{r}^{0}$. Other field quantities can be calculated in the same way, or can be derived from (6.9) by the same method as used in section 5 .

\section{The Infinitesimal Disclination Dipole}

In this section we wish to apply the general expressions of section II7 to the special case of a straight disclination dipole line. The biaxial dipole is obtained by moving the whole basic straight disclination (line and axis) by an infinitesimal distance $\xi_{m}$. The uniaxial dipole is obtained by moving only the line and keeping the axis fixed. In both cases the negative of the basic disclination is left at the $x_{3}$ axis.

\subsection{The Biaxial Dipole}

In general the defect densities of a biaxial dipole are given by, (II7.1-2),

$$
\begin{aligned}
\alpha_{p l}^{D} & =-\xi_{m} \alpha_{p l, m}, \\
\theta_{p q}^{D} & =-\xi_{m} \theta_{p q, m},
\end{aligned}
$$

where $\alpha_{p l}$ and $\theta_{p q}$ are the defect densities of the corresponding basic defect. For the special case of a discrete straight biaxial disclination dipole line the basic defect densities are given by (4.1112). So for the conjugate dipole the only nonvanishing components of $(7.1-2)$ are

$$
\begin{aligned}
\alpha_{3 l}^{D} & =-\epsilon_{l q r} \Omega_{q}\left[x_{r} \xi_{m} \delta_{, m}(\boldsymbol{\rho})+\xi_{r} \delta(\boldsymbol{\rho})\right], \\
\theta_{3 q}^{D} & =-\Omega_{q} \xi_{m} \delta, m(\boldsymbol{\rho}),
\end{aligned}
$$

where $\delta(\boldsymbol{\rho})$ is the two-dimensional Dirac delta function defined by (B9). These results could of course also be found from (II7.3-4) and (A15). In these expressions the tensor $\Omega_{q} \xi_{m}$ represents the strength of the dipole. When written out in full (7.3-4) become

$$
\begin{aligned}
& \alpha_{31}^{D}=-\Omega_{2}\left[x_{3} \xi_{1} \delta^{\prime}\left(x_{1}\right) \delta\left(x_{2}\right)+x_{3} \xi_{2} \delta\left(x_{1}\right) \delta^{\prime}\left(x_{2}\right)+\xi_{3} \delta\left(x_{1}\right) \delta\left(x_{2}\right)\right], \\
& \alpha_{32}^{D}=\Omega_{1}\left[x_{3} \xi_{1} \delta^{\prime}\left(x_{1}\right) \delta\left(x_{2}\right)+x_{3} \xi_{2} \delta\left(x_{1}\right) \delta^{\prime}\left(x_{2}\right)+\xi_{3} \delta\left(x_{1}\right) \delta\left(x_{2}\right)\right], \\
& \theta_{31}^{D}=-\Omega_{1}\left[\xi_{1} \delta^{\prime}\left(x_{1}\right) \delta\left(x_{2}\right)+\xi_{2} \delta\left(x_{1}\right) \delta^{\prime}\left(x_{2}\right)\right], \\
& \theta_{32}^{D}=-\Omega_{2}\left[\xi_{1} \delta^{\prime}\left(x_{1}\right) \delta\left(x_{2}\right)+\xi_{2} \delta\left(x_{1}\right) \delta^{\prime}\left(x_{2}\right)\right], \\
& \theta_{33}^{D}=-\Omega_{3}\left[\xi_{1} \delta^{\prime}\left(x_{1}\right) \delta\left(x_{2}\right)+\xi_{2} \delta\left(x_{1}\right) \delta^{\prime}\left(x_{2}\right)\right],
\end{aligned}
$$

where we have used (A8-9).

In general the displacement of the biaxial dipole is given by, (II7.5),

$$
u_{n}^{D}=-\xi_{m} u_{n, m}^{T}
$$

where $u_{n}^{T}$ is the displacement of the corresponding basic defect. For our special case the distortion of the basic straight disclination line is given by (5.3), and so the displacement of the conjugate 
dipole is

$$
\begin{aligned}
& u_{1}^{D}=\frac{\Omega_{1} \xi_{1} x_{3}}{4 \pi(1-\nu)}\left[(1-2 \nu) \frac{x_{1}}{\rho^{2}}-2 \frac{x_{1} x_{2}^{2}}{\rho^{4}}\right]-\frac{\Omega_{2} \xi_{2} x_{3}}{4 \pi(1-\nu)}\left[(3-2 \nu) \frac{x_{1}}{\rho^{2}}-2 \frac{x_{1} x_{2}^{2}}{\rho^{4}}\right] \\
& +\frac{\left(\Omega_{1} \xi_{2}+\Omega_{2} \xi_{1}\right) x_{3}}{4 \pi(1-\nu)}\left[(1-2 \nu) \frac{x_{2}}{\rho^{2}}+2 \frac{x_{1}^{2} x_{2}}{\rho^{4}}\right]-\left(\Omega_{2} \xi_{3}-\Omega_{3} \xi_{2}\right)\left[\frac{\varphi}{2 \pi}+\frac{x_{1} x_{2}}{4 \pi(1-\nu) \rho^{2}}\right] \\
& -\frac{\Omega_{3} \xi_{1}-\Omega_{1} \xi_{3}}{4 \pi(1-\nu)}\left[(1-2 \nu) \ln \rho+\frac{x_{2}^{2}}{\rho^{2}}\right] \\
& u_{2}^{D}=-\frac{\Omega_{1} \xi_{1} x_{3}}{4 \pi(1-\nu)}\left[(3-2 \nu) \frac{x_{2}}{\rho^{2}}-2 \frac{x_{1}^{2} x_{2}}{\rho^{4}}\right]+\frac{\Omega_{2} \xi_{2} x_{3}}{4 \pi(1-\nu)}\left[(1-2 \nu) \frac{x_{2}}{\rho^{2}}-2 \frac{x_{1}^{2} x_{2}}{\rho^{4}}\right] \\
& +\frac{\left(\Omega_{1} \xi_{2}+\Omega_{2} \xi_{1}\right) x_{3}}{4 \pi(1-\nu)}\left[(1-2 \nu) \frac{x_{1}}{\rho^{2}}+2 \frac{x_{1} x_{2}^{2}}{\rho^{4}}\right]+\frac{\Omega_{2} \xi_{3}-\Omega_{3} \xi_{2}}{4 \pi(1-\nu)}\left[(1-2 \nu) \ln \rho+\frac{x_{1}^{2}}{\rho^{2}}\right] \\
& -\left(\Omega_{3} \xi_{1}-\Omega_{1} \xi_{3}\right)\left[\frac{\varphi}{2 \pi}-\frac{x_{1} x_{2}}{4 \pi(1-\nu) \rho^{2}}\right] \\
& u_{3}^{D}=\frac{\Omega_{1} \xi_{1}}{4 \pi(1-\nu)}\left[(1-2 \nu) \ln \rho+\frac{x_{2}^{2}}{\rho^{2}}\right]+\frac{\Omega_{2} \xi_{2}}{4 \pi(1-\nu)}\left[(1-2 \nu) \ln \rho+\frac{x_{1}^{2}}{\rho^{2}}\right] \\
& -\left(\Omega_{1} \xi_{2}-\Omega_{2} \xi_{1}\right)\left[\frac{\varphi}{2 \pi}-\frac{x_{1} x_{2}}{4 \pi(1-\nu) \rho^{2}}\right]
\end{aligned}
$$

where we have ignored the terms in (5.3) which are concentrated on the surface $S$. We presume that the displacement is not a state quantity because it contains the angle $\phi$. The displacement has a logarithmic dependence at large distances from the dipole line, in agreement with table II3.

\subsection{The Uniaxial Dipole}

To derive the relations for the uniaxial disclination dipole we first find the results of moving the axis of the basic disclination line by an infinitesimal distance $\xi_{m}$, keeping the disclination line fixed. The dislocation density of this defect is given by, (II7.7),

$$
\alpha_{p l}^{A}=\xi_{m} \partial \alpha_{p l} / \partial x_{m}^{0},
$$

whereas the disclination density vanishes, $\theta_{p q}^{A}=0$. For our special case of a straight disclination line we find from (6.1) that the only nonvanishing components of (7.7) are

$$
\alpha_{3 l}^{A}=-\epsilon_{l q r} \Omega_{q} \xi_{r} \delta(\boldsymbol{\rho}),
$$

in agreement with (II7.9) and (A15). The displacement of the above defect is, (II7.11),

$$
u_{n}^{A}=\xi_{m} \partial u_{n}^{T} / \partial x_{m}^{0},
$$

which, for our special case, becomes

$$
u_{1}^{A}=-\left(\Omega_{2} \xi_{3}-\Omega_{3} \xi_{2}\right)\left[\frac{\varphi}{2 \pi}+\frac{x_{1} x_{2}}{4 \pi(1-\nu) \rho^{2}}\right]-\frac{\Omega_{3} \xi_{1}-\Omega_{1} \xi_{3}}{4 \pi(1-\nu)}\left[(1-2 \nu) \ln \rho+\frac{x_{2}}{\rho^{2}}\right],
$$




$$
\begin{aligned}
& u_{2}^{A}=\frac{\Omega_{2} \xi_{3}-\Omega_{3} \xi_{2}}{4 \pi\left(1-\nu^{\prime}\right)}\left[(1-2 \nu) \ln \rho+\frac{x_{1}^{2}}{\rho^{2}}\right]-\left(\Omega_{3} \xi_{1}-\Omega_{1} \xi_{3}\right)\left[\frac{\varphi}{2 \pi}-\frac{x_{1} x_{2}}{4 \pi(1-\nu) \rho^{2}}\right] \\
& u_{3}^{A}=-\left(\Omega_{1} \xi_{2}-\Omega_{2} \xi_{1}\right) \frac{\varphi}{2 \pi}
\end{aligned}
$$

from (6.9). If we compare (7.8) and (7.10) with (3.4) and (3.12) we see that the above results are the dislocation density and displacement of a discrete straight dislocation line with a Burgers vector given by

$$
b_{l}=-\epsilon_{l q r} \Omega_{q} \xi_{r}
$$

in agreement with (II7.13).

We now find the relations for the discrete straight uniaxial disclination dipole line. In general they are given by the difference between those in section 7.1 and the above. For example, the dislocation density is found from, (II7.14),

$$
\alpha_{p l}^{L}=\alpha_{p l}^{D}-\alpha_{p l}^{A}
$$

Hence from (7.3) and (7.8) we find that the only nonvanishing components of (7.12) are

$$
\alpha_{3 l}^{L}=-\epsilon_{l q r} \Omega_{q} x_{r} \xi_{m} \delta, m(\boldsymbol{\rho}),
$$

in agreement with (II7.15) and (A15). When written out in full this expression becomes

$$
\begin{aligned}
& \alpha_{31}^{L}=-\Omega_{2} x_{3}\left[\xi_{1} \delta^{\prime}\left(x_{1}\right) \delta\left(x_{2}\right)+\xi_{2} \delta\left(x_{1}\right) \delta^{\prime}\left(x_{2}\right)\right]-\Omega_{3} \xi_{2} \delta\left(x_{1}\right) \delta\left(x_{2}\right), \\
& \alpha_{32}^{L}=\Omega_{1} x_{3}\left[\xi_{1} \delta^{\prime}\left(x_{1}\right) \delta\left(x_{2}\right)+\xi_{2} \delta\left(x_{1}\right) \delta^{\prime}\left(x_{2}\right)\right]+\Omega_{3} \xi_{1} \delta\left(x_{1}\right) \delta\left(x_{2}\right), \\
& \alpha_{33}^{L}=\left(\Omega_{1} \xi_{2}-\Omega_{2} \xi_{1}\right) \delta\left(x_{1}\right) \delta\left(x_{2}\right),
\end{aligned}
$$

where we have used (A8-9). The disclination density of the uniaxial dipole is the same as for the biaxial dipole, $\theta_{p q}^{L}=\theta_{p q}^{D}$, given by (7.4). The displacement of the uniaxial dipole is given by, (II7.16),

$$
u_{n}^{L}=u_{n}^{D}-u_{n}^{A}
$$

By (7.6) and (7.10) this becomes for our special case

$$
\begin{array}{r}
u_{1}^{L}=\frac{\Omega_{1} \xi_{1} x_{3}}{4 \pi(1-\nu)}\left[(1-2 \nu) \frac{x_{1}}{\rho^{2}}-2 \frac{x_{1} x_{2}^{2}}{\rho^{4}}\right]-\frac{\Omega_{2} \xi_{2} x_{3}}{4 \pi(1-\nu)}\left[(3-2 \nu) \frac{x_{1}}{\rho^{2}}-2 \frac{x_{1} x_{2}^{2}}{\rho^{4}}\right] \\
\quad+\frac{\left(\Omega_{1} \xi_{2}+\Omega_{2} \xi_{1}\right) x_{3}}{4 \pi(1-\nu)}\left[(1-2 \nu) \frac{x_{2}}{\rho^{2}}+2 \frac{x_{1}^{2} x_{2}}{\rho^{4}}\right], \\
\begin{array}{r}
u_{2}^{L}=-\frac{\Omega_{1} \xi_{1} x_{3}}{4 \pi(1-\nu)}\left[(3-2 \nu) \frac{x_{2}}{\rho^{2}}-2 \frac{x_{1}^{2} x_{2}}{\rho^{4}}\right]+\frac{\Omega_{2} \xi_{2} x_{3}}{4 \pi(1-\nu)}\left[(1-2 \nu) \frac{x_{2}}{\rho^{2}}-2 \frac{x_{1}^{2} x_{2}}{\rho^{4}}\right] \\
+\frac{\left(\Omega_{1} \xi_{2}+\Omega_{2} \xi_{1}\right) x_{3}}{4 \pi(1-\nu)}\left[(1-2 \nu) \frac{x_{1}}{\rho^{2}}+2 \frac{x_{1} x_{2}^{2}}{\rho^{4}}\right],
\end{array}
\end{array}
$$




$$
\begin{array}{r}
u_{3}^{L}=\frac{\Omega_{1} \xi_{1}}{4 \pi(1-\nu)}\left[(1-2 \nu) \ln \rho+\frac{x_{2}^{2}}{\rho^{2}}\right]+\frac{\Omega_{2} \xi_{2}}{4 \pi(1-\nu)}\left[(1-2 \nu) \ln \rho+\frac{x_{1}^{2}}{\rho^{2}}\right] \\
+\left(\Omega_{1} \xi_{2}-\Omega_{2} \xi_{1}\right) \frac{x_{1} x_{2}}{4 p(1-\nu) \rho^{2}} .
\end{array}
$$

We note that these expressions no longer contain the angle $\varphi$, and so $u_{n}^{L}$ is a state quantity. The asymptotic dependence at large distances is the same as the biaxial dipole, and shown in table II3.

Furthermore the displacement of the uniaxial dipole $u_{n}^{L}$ does not contain any contribution from the wedge disclination, i.e., $\Omega_{3}$ is missing from (7.15). We may therefore conclude that it is possible to find the displacements of an edge dislocation from those of a biaxial wedge disclination dipole. Specifically if we set

$$
\Omega_{1}=\Omega_{2}=0,
$$

we find for (7.11)

$$
\begin{aligned}
& b_{1}=\Omega_{3} \xi_{2}, \\
& b_{2}=-\Omega_{3} \xi_{1} .
\end{aligned}
$$

If these results are substituted in $(7.6 \mathrm{a}-\mathrm{b})$ we find $(3.12 \mathrm{a}-\mathrm{b})$. This method of obtaining the edge dislocation displacements was first pointed out by Eshelby [11].

\section{The "Dislocation Model" of the Straight Disclination}

\subsection{Geometry}

As discussed in section II9 the dislocation model of a discrete defect line along the curve $L$ is obtained by eliminating the disclination loop density (II9.1)

$$
\varphi_{k q}^{*}=0,
$$

from the corresponding defect surface $S$ that spans $L$. We shall illustrate this for our special case and so find the dislocation model of a straight disclination. There are of course many possible choices for the surface $S$, but we consider only the one used in the previous sections and illustrated in figure 1.

The dislocation loop density remains the same as before, (4.3), and becomes the plastic distortion, (II9.2). So it has the only nonvanishing components

$$
\beta_{2 l}^{P}=\beta_{2 l}^{*}=\epsilon_{l q r} \Omega_{q} x_{r} H\left(-x_{1}\right) \delta\left(x_{2}\right),
$$

or, when written out in full

$$
\begin{aligned}
& \beta_{21}^{P}=\beta_{21}^{*}=\Omega_{2} x_{3} H\left(-x_{1}\right) \delta\left(x_{2}\right), \\
& \beta_{22}^{P}=\beta_{22}^{*}=\left(\Omega_{3} x_{1}-\Omega_{1} x_{3}\right) H\left(-x_{1}\right) \delta\left(x_{2}\right), \\
& \beta_{23}^{P}=\beta_{23}^{*}=-\Omega_{2} x_{1} H\left(-x_{1}\right) \delta\left(x_{3}\right) .
\end{aligned}
$$

The dislocation density of the dislocation model is in general given by, (II9.7), 


$$
\alpha_{p l}(\mathbf{r})=\delta_{p}(L)\left\{b_{l}+\epsilon_{l q r} \Omega_{q}\left(x_{r}-x_{r}^{0}\right)\right\}+\delta_{l}(S) \Omega_{p}-\delta_{p l} \delta_{k}(S) \Omega_{k} .
$$

For our special case, with $b_{l}=x_{r}^{0}=0$, this reduces to

$$
\begin{aligned}
& \alpha_{11}=\Omega_{2} H\left(-x_{1}\right) \delta\left(x_{2}\right), \\
& \alpha_{21}=0, \\
& \alpha_{31}=\Omega_{2} x_{3} \delta\left(x_{1}\right) \delta\left(x_{2}\right), \\
& \alpha_{12}=-\Omega_{1} H\left(-x_{1}\right) \delta\left(x_{2}\right), \\
& \alpha_{22}=0, \\
& \alpha_{32}=-\Omega_{1} x_{3} \delta\left(x_{1}\right) \delta\left(x_{2}\right)-\Omega_{3} H\left(-x_{1}\right) \delta\left(x_{2}\right), \\
& \alpha_{13}=0, \\
& \alpha_{23}=0, \\
& \alpha_{33}=\Omega_{2} H\left(-x_{1}\right) \delta\left(x_{2}\right),
\end{aligned}
$$

where we have used (A15-16) and (A8). The dislocation model consists of two parts, the same dislocation density along $L$ as for the corresponding discrete disclination, given by (4.11), and a constant distribution over the surface $S$, given by the terms with the Heaviside function. We have sketched the three different type of dislocation models for $\Omega_{1}, \Omega_{2}$, and $\Omega_{3}$ in figures 9-11. Rather than the constant dislocation distribution over $S$ implied by (8.4), which is somewhat difficult to draw, we have shown the discrete distribution that most closely approximates it, and is somewhat easier to visualize. These figures represent the dislocation models of the straight disclinations shown in figures 6-8. Figures 9 and 11 represent terminating tilt walls and figure 10 a terminating twist wall. The disclination density of course vanishes, (II9.8),

$$
\theta_{p q}=0 .
$$

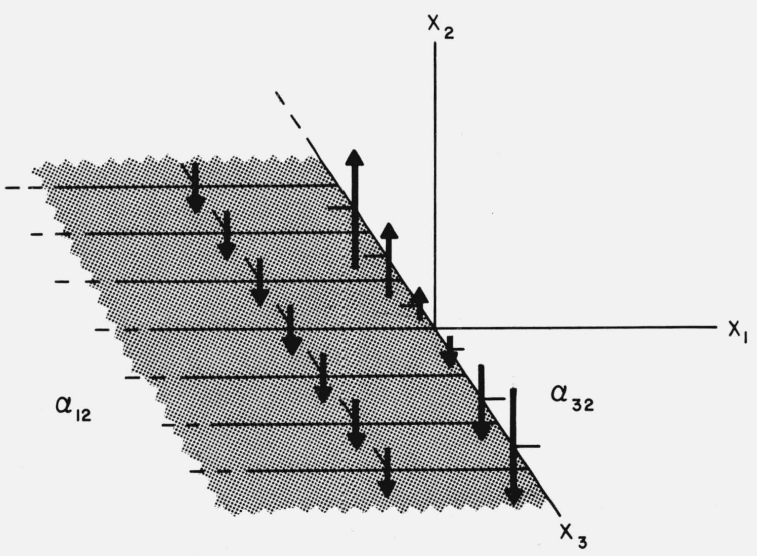

Figure 9. Dislocation model of the twist disclination in figure 6 .

The tilt wall on the surface $S$ (fig. 1), which terminates at the $x_{3}$ axis, is represented by a set of parallel edge dislocations. The arrows are Burgers vectors and the attached short lines show the orientation of the extra half planes. This defect is described by the dislocation densities $\alpha_{12}=-\Omega_{1} H\left(-x_{1}\right) \delta\left(x_{2}\right)$ and $\alpha_{32}=-$ $\Omega_{1} x_{3} \delta\left(x_{1}\right) \delta\left(x_{2}\right)$.

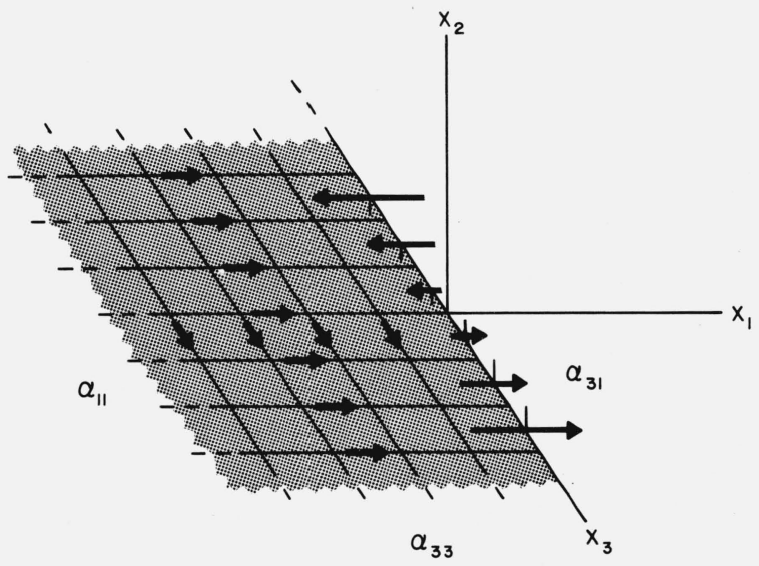

FIGURE 10. Dislocation model of the twist disclination in figure 7.

The twist wall on the surface $S$, which terminates at the $x_{3}$ axis, is represented by a crossed grid of screw dislocations. The arrows are Burgers vectors and the attached short lines show the orientation of the extra half planes for the edge dislocations. This defect is described by the dislocation densities $\alpha_{11}=\alpha_{33}=\Omega_{2} H$ $\left(-x_{1}\right) \delta\left(x_{2}\right)$ and $\alpha_{31}=\Omega_{2} x_{3} \delta\left(x_{1}\right) \delta\left(x_{2}\right)$. 


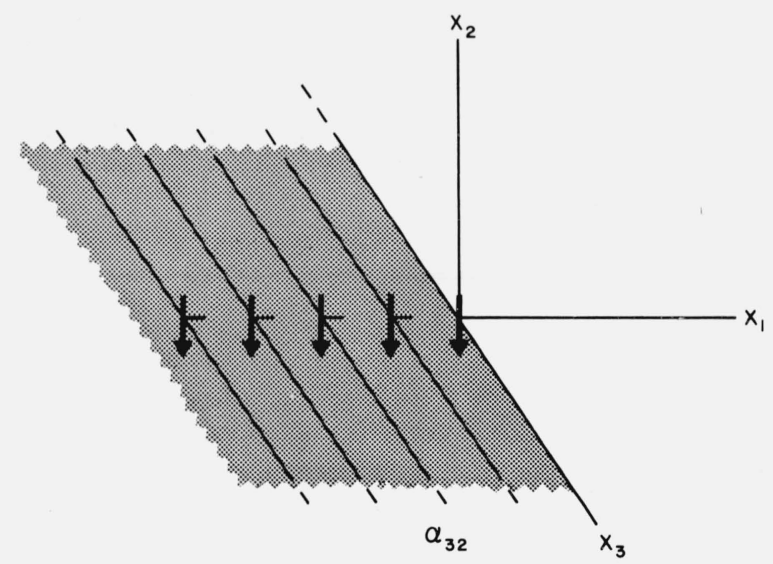

FIGURE 11. Dislocation model of the wedge disclination in figure 8.

The tilt wall on the surface $S$, which terminates at the $x_{3}$ axis, is represented by a set of parallel edge dislocations. The arrows are Burgers vectors and the attached short lines show the orientation of the extra half planes. This defect is described by the dislocation density $\alpha_{32}=-\Omega_{3} H\left(-x_{1}\right) \delta\left(x_{2}\right)$.

For consistency it is easily verified that (8.2) and (8.4) satisfy the definition (3.5). It is also easily checked that the continuity condition (3.6) is satisfied by (8.4), in agreement with (II9.9).

We conclude this section with expressions for the incompatibility tensor. If we substitute (8.4) into (3.9) we find the only nonvanishing components

$$
\begin{aligned}
& \eta_{13}=-\Omega_{1} \delta\left(x_{1}\right) \delta\left(x_{2}\right), \\
& \eta_{23}=-\Omega_{2} \delta\left(x_{1}\right) \delta\left(x_{2}\right), \\
& \eta_{33}=\Omega_{1} x_{3} \delta^{\prime}\left(x_{1}\right) \delta\left(x_{2}\right)+\Omega_{2} x_{3} \delta\left(x_{1}\right) \delta^{\prime}\left(x_{2}\right)-\Omega_{3} \delta\left(x_{1}\right) \delta\left(x_{2}\right) .
\end{aligned}
$$

These results are identical with the incompatibility tensor of the corresponding disclination given by (4.31). Since the incompatibility is the source of elastic strain, (II4.22) and (II4.31), this means that the dislocation model has the same elastic strain as the corresponding discrete disclination line.

\subsection{Statics}

According to (II9.11-12) the total displacement and distortion of the dislocation model are identical to those for the corresponding disclination line. They are therefore given by (5.1) and (5.3).

To determine the elastic fields it is necessary to know how to split up the total fields. The difference between a discrete disclination line and its dislocation model simply results from splitting the total fields up in a different way. For a discrete disclination we have argued that the total distortion cannot be split into an elastic and plastic part. In particular in section 5 we argued that for the straight disclination (5.3) cannot be split because it contains the angle $\varphi$. For the dislocation model, however, we ignore this point of view and say that the total distortion can be split into an elastic and plastic part. This approach leads to Mura's new concepts of "plastic distortion" and "elastic distortion" of a disclination line. In particular, for the straight disclination, we assert that (5.3) can be split. The logical terms for the plastic part are those that are concentrated on the surface $S$, which correspond exactly to (8.2), or the dislocation loop density of section 4 . So we find the elastic distortion from (3.13), (5.3), and (8.2), 


$$
\begin{aligned}
& \beta_{11}=-\frac{\Omega_{1} x_{3}}{4 \pi(1-\nu)}\left[(1-2 \nu) \frac{x_{1}}{\rho^{2}}-2 \frac{x_{1} x_{2}^{2}}{\rho^{4}}\right]-\frac{\Omega_{2} x_{3}}{4 \pi(1-\nu)}\left[(1-2 \nu) \frac{x_{2}}{\rho^{2}}+2 \frac{x_{1}^{2} x_{2}}{\rho^{4}}\right] \\
& +\frac{\Omega_{3}}{4 \pi(1-\nu)}\left[(1-2 \nu) \ln \rho+\frac{x_{2}^{2}}{\rho^{2}}\right], \\
& \beta_{21}=-\frac{\Omega_{1} x_{3}}{4 \pi(1-\nu)}\left[(1-2 \nu) \frac{x_{2}}{\rho^{2}}+2 \frac{x_{1}^{2} x_{2}}{\rho^{4}}\right]+\frac{\Omega_{2} x_{3}}{4 \pi(1-\nu)}\left[(3-2 \nu) \frac{x_{1}}{\rho^{2}}-2 \frac{x_{1} x_{2}^{2}}{\rho^{4}}\right] \\
& -\Omega_{3}\left[\frac{\varphi}{2 \pi}+\frac{x_{1} x_{2}}{4 \pi(1-\nu) \rho^{2}}\right] \text {, } \\
& \beta_{31}=-\frac{\Omega_{1}}{4 \pi(1-\nu)}\left[(1-2 \nu) \ln \rho+\frac{x_{2}^{2}}{\rho^{2}}\right]+\Omega_{2}\left[\frac{\varphi}{2 \pi}+\frac{x_{1} x_{2}}{4 \pi(1-\nu) \rho^{2}}\right], \\
& \beta_{12}=\frac{\Omega_{1} x_{3}}{4 \pi(1-\nu)}\left[(3-2 \nu) \frac{x_{2}}{\rho^{2}}-2 \frac{x_{1}^{2} x_{2}}{\rho^{4}}\right]-\frac{\Omega_{2} x_{3}}{4 \pi(1-\nu)}\left[(1-2 \nu) \frac{x_{1}}{\rho^{2}}+2 \frac{x_{1} x_{2}^{2}}{\rho^{4}}\right] \\
& +\Omega_{3}\left[\frac{\varphi}{2 \pi}-\frac{x_{1} x_{2}}{4 \pi(1-\nu) \rho^{2}}\right] \\
& \beta_{22}=-\frac{\Omega_{1} x_{3}}{4 \pi(1-\nu)}\left[(1-2 \nu) \frac{x_{1}}{\rho^{2}}+2 \frac{x_{1} x_{2}^{2}}{\rho^{4}}\right]-\frac{\Omega_{2} x_{3}}{4 \pi(1-\nu)}\left[(1-2 \nu) \frac{x_{2}}{\rho^{2}}-2 \frac{x_{1}^{2} x_{2}}{\rho^{4}}\right] \\
& +\frac{\Omega_{3}}{4 \pi(1-\nu)}\left[(1-2 \nu) \ln \rho+\frac{x_{1}^{2}}{\rho^{2}}\right] \text {, } \\
& \beta_{32}=-\Omega_{1}\left[\frac{\varphi}{2 \pi}-\frac{x_{1} x_{2}}{4 \pi(1-\nu) \rho^{2}}\right]-\frac{\Omega_{2}}{4 \pi(1-\nu)}\left[(1-2 \nu) \ln \rho+\frac{x_{1}^{2}}{\rho^{2}}\right], \\
& \beta_{13}=-\frac{\Omega_{1}}{4 \pi(1-\nu)}\left[(1-2 \nu) \ln \rho+\frac{x_{2}^{2}}{\rho^{2}}\right]-\Omega_{2}\left[\frac{\varphi}{2 \pi}-\frac{x_{1} x_{2}}{4 \pi(1-\nu) \rho^{2}}\right], \\
& \beta_{23}=\Omega_{1}\left[\frac{\varphi}{2 \pi}+\frac{x_{1} x_{2}}{4 \pi(1-\nu) \rho^{2}}\right]-\frac{\Omega_{2}}{4 \pi(1-\nu)}\left[(1-2 \nu) \ln \rho+\frac{x_{1}^{2}}{\rho^{2}}\right], \\
& \beta_{33}=0 \text {. }
\end{aligned}
$$

This is the elastic distortion of the "dislocation model" of the straight disclination line. It also represents Mura's "elastic distortion" of the straight disclination line. Note that these expressions still contain the angle $\phi$. For consistency it is straightforward to check that (8.7) and (8.4) satisfy the field equation (3.15). From the elastic distortion we can then find all the other elastic fields of the dislocation model.

The elastic strain is obtained by substituting (8.7) into (3.16) and the result is identical to (5.5). Thus the elastic strain and hence stress of the dislocation model is identical to that of the corresponding discrete disclination, as we already discussed in section II9.

The elastic rotation is found from (3.22) and (8.7)

$$
\begin{aligned}
& \omega_{1}=\frac{\Omega_{1} \varphi}{2 \pi}, \\
& \omega_{2}=\frac{\Omega_{2} \varphi}{2 \pi},
\end{aligned}
$$




$$
\omega_{3}=\frac{\Omega_{1} x_{2} x_{3}}{2 \pi \rho^{2}}-\frac{\Omega_{2} x_{1} x_{3}}{2 \pi \rho^{2}}+\frac{\Omega_{3} \varphi}{2 \pi} .
$$

In general the elastic bend-twist is given by, (II3.14),

$$
\kappa_{k q}=\omega_{q, k} .
$$

For our special case (8.8) we find the components

$$
\begin{aligned}
& \kappa_{11}=-\frac{\Omega_{1} x_{2}}{2 \pi \rho^{2}}, \\
& \kappa_{21}=\frac{\Omega_{1} x_{1}}{2 \pi \rho^{2}}+\Omega_{1} H\left(-x_{1}\right) \delta\left(x_{2}\right), \\
& \kappa_{31}=0, \\
& \kappa_{12}=-\frac{\Omega_{1} x_{2}}{2 \pi \rho^{2}}, \\
& \kappa_{22}=-\frac{\Omega_{2} x_{1}}{2 \pi \rho^{2}}+\Omega_{2} H\left(-x_{1}\right) \delta\left(x_{2}\right), \\
& \kappa_{32}=0, \\
& \kappa_{13}=-\frac{\Omega_{1} x_{1} x_{2} x_{3}}{\pi \rho^{4}}-\frac{\Omega_{2} x_{3}}{2 \pi}\left[\frac{1}{\rho^{2}}-2 \frac{x_{1}^{2}}{\rho^{4}}+\pi \delta(\boldsymbol{\rho})\right]-\frac{\Omega_{3} x_{2}}{2 \pi \rho^{2}}, \\
& \kappa_{23}=\frac{\Omega_{1} x_{3}}{2 \pi}\left[\frac{1}{\rho^{2}}-2_{2} \frac{x_{2}^{2}}{\rho^{4}}+\pi \delta(\boldsymbol{\rho})\right]+\frac{\Omega_{2} x_{1} x_{2} x_{3}}{\pi \rho^{4}} \\
& \kappa_{33}=\frac{\Omega_{1} x_{2}}{2 \pi \rho^{2}}-\frac{\Omega_{2} x_{1}}{2 \pi \rho^{2}},
\end{aligned}
$$

where we have used (B3-4) and (B8). The elastic bend-twist of the dislocation model consists of the sum of the elastic bend-twist of the corresponding disclination line, (5.9), and the disclination loop density or Mura's "plastic rotation," (4.4). For consistency it can easily be verified that (8.10) and (8.5) satisfy the field equation (5.11), by (B8) and (B11-12).

Mura's new concepts therefore have a clear interpretation in terms of the dislocation model. Mura's "plastic and elastic distortion" of the disclination line are the plastic and elastic distortion of the corresponding dislocation model. Mura's "plastic rotation" is the excess elastic bend-twist of the dislocation model over the corresponding disclination line.

\section{The Finite Disclination Dipole}

The results for a finite dipole can be derived simply by combining the fields of a positive and negative basic disclination which are a finite distance apart. We choose the positive disclination to pass through the point $(L, 0,0)$ and the negative one through $(-L, 0,0)$, both parallel to the $x_{3}$ axis.

\subsection{The Biaxial Dipole}

a. Geometry

We find the defect densities for the finite straight biaxial disclination dipole from the basic defect densities of the discrete straight disclination line, given by (4.11-12), using the above pro- 
cedure. The only nonvanishing components are

$$
\begin{aligned}
& \alpha_{31}^{D}=\Omega_{2} x_{3}\left[\delta\left(x_{1}-L\right)-\delta\left(x_{1}+L\right)\right] \delta\left(x_{2}\right), \\
& \alpha_{32}^{D}=-\Omega_{1} x_{3}\left[\delta\left(x_{1}-L\right)-\delta\left(x_{1}+L\right)\right] \delta\left(x_{2}\right), \\
& \theta_{3 q}^{D}=\Omega_{q}\left[\delta\left(x_{1}-L\right)-\delta\left(x_{1}+L\right)\right] \delta\left(x_{2}\right) .
\end{aligned}
$$

It is interesting also to find the dislocation model for this defect since it has exactly the same total displacement, total distortion, elastic strain, and stress. The defect densities for the dislocation model of the finite biaxial disclination dipole can be found from the basic defect densities of the dislocation model of the discrete straight disclination line, given by (8.4-5). Using the same procedure again, we find

$$
\begin{aligned}
& \alpha_{11}^{D}=-\Omega_{2}\left[H\left(x_{1}-L\right)-H\left(x_{1}+L\right)\right] \delta\left(x_{2}\right), \\
& \alpha_{21}^{D}=0, \\
& \alpha_{31}^{D}=\Omega_{2} x_{3}\left[\delta\left(x_{1}-L\right)-\delta\left(x_{1}+L\right)\right] \delta\left(x_{2}\right), \\
& \alpha_{12}^{D}=\Omega_{1}\left[H\left(x_{1}-L\right)-H\left(x_{2}+L\right)\right] \delta\left(x_{2}\right), \\
& \alpha_{22}^{D}=0, \\
& \alpha_{32}^{D}=-\Omega_{1} x_{3}\left[\delta\left(x_{1}-L\right)-\delta\left(x_{1}+L\right)\right] \delta\left(x_{2}\right)+\Omega_{3}\left[H\left(x_{1}-L\right)-H\left(x_{1}+L\right)\right] \delta\left(x_{2}\right), \\
& \alpha_{13}^{D}=0, \\
& \alpha_{23}^{D}=0, \\
& \alpha_{33}^{D}=-\Omega_{2}\left[H\left(x_{1}-L\right)-H\left(x_{1}+L\right)\right] \delta\left(x_{2}\right), \\
& \theta_{p q}^{D}=0 .
\end{aligned}
$$

The dislocation model consists of two parts: the same dislocation density as for the corresponding defect, given by (9.1), and a constant distribution over the surface strip between $(-L, 0,0)$ and $(L, 0,0)$. We have illustrated this case in figures 12-14. The dislocation model of the biaxial wedge disclination dipole, figure 14, was examined by $\mathrm{Li}$ [12], who called it a "dislocation wall."

\section{b. Statics}

The fields of the finite straight biaxial disclination dipole can be found from the fields of the corresponding basic defect, i.e., the straight disclination line, given in section 5 , by the same procedure as used above. We shall illustrate this by two examples. We first find the total displacement of the biaxial disclination dipole from (5.1)

$$
\begin{aligned}
u_{1}^{D}= & -\frac{\Omega_{1} x_{3}}{4 \pi(1-\nu)}\left[\frac{1}{2}(1-2 \nu) \ln \frac{\left(x_{1}-L\right)^{2}+x_{2}^{2}}{\left(x_{1}+L\right)^{2}+x_{2}^{2}}+\frac{x_{2}^{2}}{\left(x_{1}-L\right)^{2}+x_{2}^{2}}-\frac{x_{2}^{2}}{\left(x_{1}+L\right)^{2}+x_{2}^{2}}\right] \\
& +\frac{\Omega_{2} x_{3}}{2 \pi}\left[\varphi\left(x_{1}-L, x_{2}\right)-\varphi\left(x_{1}+L, x_{2}\right)+\frac{x_{2}}{2(1-\nu)}\left\{\frac{x_{1}-L}{\left(x_{1}-L\right)^{2}+x_{2}^{2}}-\frac{x_{1}+L}{\left(x_{1}+L\right)^{2}+x_{2}^{2}}\right\}\right]
\end{aligned}
$$




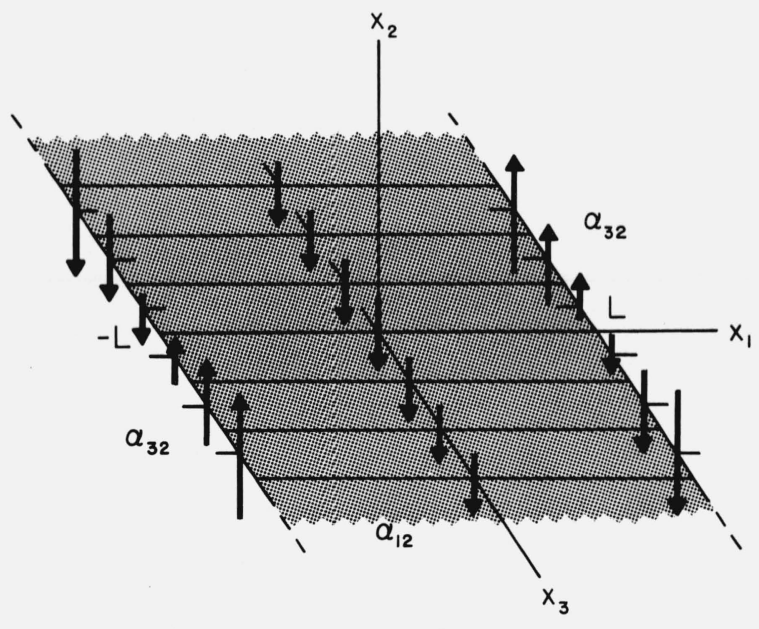

Figure 12. Dislocation model of the finite twist disclination dipole (biaxial and uniaxial), corresponding to figures 6 and 9.

The dislocation densities are $\alpha_{12}^{D}=\Omega_{1}\left[H\left(x_{1}-L\right)-H\left(x_{2}+L\right)\right] \delta\left(x_{2}\right)$ and $\alpha_{32}^{D}=$ $-\Omega_{1} x_{3}\left[\delta\left(x_{1}-L\right)-\delta\left(x_{1}+L\right)\right] \delta\left(x_{2}\right)$.

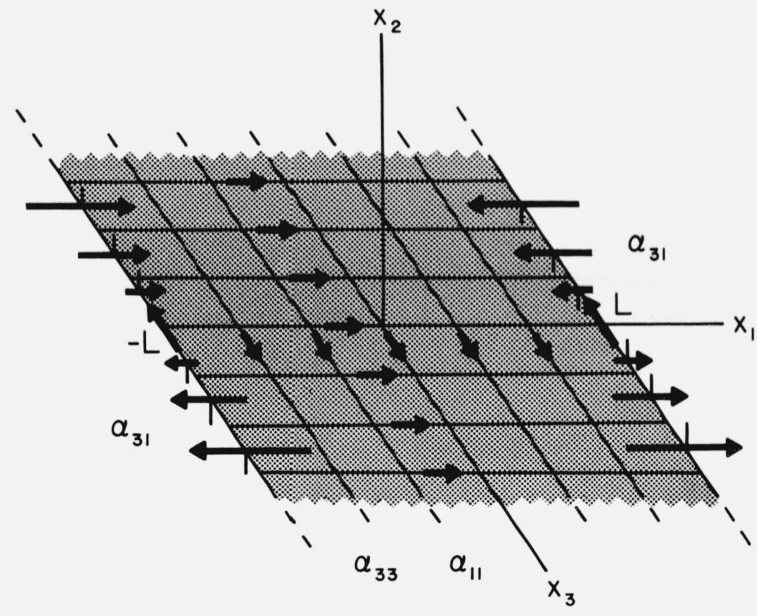

FigURE 13. Dislocation model of the finite twist disclination dipole, corresponding to figures 7 and 10.

The dislocation densities for the biaxial dipole are $\alpha_{11}^{D}=\alpha_{33}^{D}=-\Omega_{2}\left[H\left(x_{1}-L\right)\right.$ $\left.-H\left(x_{1}+L\right)\right] \delta\left(x_{2}\right)$ and $\alpha_{31}^{D=} \Omega_{2} x_{3}\left[\delta\left(x_{1}-L\right)-\delta\left(x_{1}+L\right)\right] \delta\left(x_{2}\right)$. For the uniaxial dipole we have to add the dislocation density $\alpha_{33}^{A}=-\Omega_{2} L\left[\delta\left(x_{1}-L\right)+\delta\left(x_{1}+L\right)\right]$ $\delta\left(x_{2}\right)$, which is indicated by the two Burgers vectors at $L$ and $-L$.

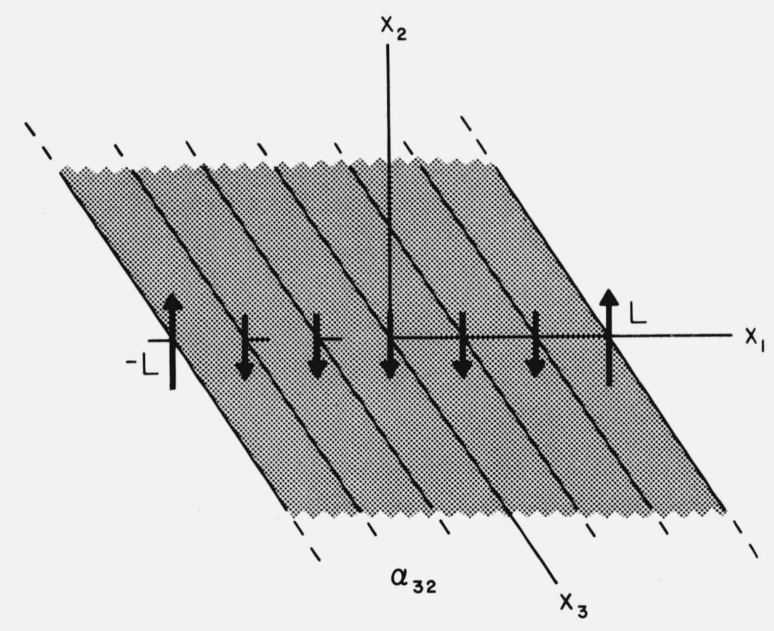

FIGURE 14. Disclination model of the finite wedge disclination dipole, corresponding to figures 8 and 11.

The dislocation density for the biaxial dipole is $\alpha^{D}=\Omega_{3}\left[H\left(x_{1}-L\right)-H\left(x_{1}+\right.\right.$ $L)] \delta\left(x_{2}\right)$. For the uniaxial dipole we have to add the dislocation density $\alpha_{32}^{A}=$ $\Omega_{3} L\left[\delta\left(x_{1}-L\right)+\delta\left(x_{1}+L\right)\right] \delta\left(x_{2}\right)$, which is indicated by the two large Burgers vectors at $L$ and $-L$.

$$
\begin{gathered}
-\frac{\Omega_{3}}{2 \pi}\left[x_{2}\left\{\varphi\left(x_{1}-L, x_{2}\right)-\varphi\left(x_{1}+L, x_{2}\right)\right\}-\frac{1-2 \nu}{2(1-\nu)}\left\{\frac{1}{2} x_{1} \ln \frac{\left(x_{1}-L\right)^{2}+x_{2}^{2}}{\left(x_{1}+L\right)^{2}+x_{2}^{2}}\right.\right. \\
\left.\left.-\frac{1}{2} L \ln \left(\left(x_{1}-L\right)^{2}+x_{2}^{2}\right)\left(\left(x_{1}+L\right)^{2}+x_{2}^{2}\right)+2 L\right\}\right], \\
u_{2}^{D}=-\frac{\Omega_{1} x_{3}}{2 \pi}\left[\varphi\left(x_{1}-L, x_{2}\right)-\varphi\left(x_{1}+L, x_{2}\right)-\frac{x_{2}}{2(1-\nu)}\left\{\frac{x_{1}-L}{\left(x_{1}-L\right)^{2}+x_{2}^{2}}-\frac{x_{1}+L}{\left(x_{1}+L\right)^{2}+x_{2}^{2}}\right\}\right] \\
-\frac{\Omega_{2} x_{3}}{4 \pi(1-\nu)}\left[\frac{1}{2}(1-2 \nu) \ln \frac{\left(x_{1}-L\right)^{2}+x_{2}^{2}}{\left(x_{1}+L\right)^{2}+x^{2}}+\frac{\left(x_{1}-L\right)^{2}}{\left(x_{1}-L\right)^{2}+x_{2}^{2}}-\frac{\left(x_{1}+L\right)^{2}}{\left(x_{1}+L\right)^{2}+x_{2}^{2}}\right]
\end{gathered}
$$




$$
\begin{array}{r}
+\frac{\Omega_{3}}{2 \pi}\left[x_{1}\left\{\varphi\left(x_{1}-L, x_{2}\right)-\varphi\left(x_{1}+L, x_{2}\right)\right\}-\frac{1}{2}\left\{\varphi\left(x_{1}-L, x_{2}\right)+\varphi\left(x_{1}+L, x_{2}\right)\right\}\right. \\
\left.+\frac{1-2 \nu}{2(1-\nu)} x_{2} \frac{1}{2} \ln \frac{\left(x_{1}-L\right)^{2}+x_{2}^{2}}{\left(x_{1}+L\right)^{2}+x_{2}^{2}}\right], \\
u_{3}^{D}=\frac{\Omega_{1}}{2 \pi}\left[x_{2}\left\{\varphi\left(x_{1}-L, x_{2}\right)-\varphi\left(x_{1}+L, x_{2}\right)\right\}-\frac{1-2 \nu}{2(1-\nu)}\left\{\frac{1}{2} x_{1} \ln \frac{\left(x_{1}-L\right)^{2}+x_{2}^{2}}{\left(x_{1}+L\right)^{2}+x_{2}^{2}}\right.\right. \\
\left.\left.-\frac{1}{2} L \ln \left(\left(x_{1}-L\right)^{2}+x_{2}^{2}\right)\left(\left(x_{1}+L\right)^{2}+x_{2}^{2}\right)+2 L\right\}\right]-\frac{\Omega_{2}}{2 \pi}\left[x_{1}\left\{\varphi\left(x_{1}-L, x_{2}\right)-\varphi\left(x_{1}+L, x_{2}\right)\right\}\right. \\
\left.-L\left\{\varphi\left(x_{1}-L, x_{2}\right)+\varphi\left(x_{1}+L, x_{2}\right)\right\}+\frac{1-2 \nu}{2(1-\nu)} x_{2}-\frac{1}{2} \ln \frac{\left(x_{1}-L\right)^{2}+x_{2}^{2}}{\left(x_{1}+L\right)^{2}+x_{2}^{2}}\right],
\end{array}
$$

where $\varphi\left(x_{1}, x_{2}\right)$ is defined by (B2). Second we find the stress of the biaxial wedge disclination dipole from (5.12), setting $\Omega_{1}=\Omega_{2}=0$,

$$
\begin{aligned}
& \sigma_{11}^{D}=\frac{G \Omega_{3}}{2 \pi(1-\nu)}\left[\frac{1}{2} \ln \frac{\left(x_{1}-L\right)^{2}+x_{2}^{2}}{\left(x_{1}+L\right)^{2}+x_{2}^{2}}+\frac{x_{2}^{2}}{\left(x_{1}-L\right)^{2}+x_{2}^{2}}-\frac{x_{2}^{2}}{\left(x_{1}+L\right)^{2}+x_{2}^{2}}\right] \\
& \sigma_{22}^{D}=\frac{G \Omega_{3}}{2 \pi(1-\nu)}\left[\frac{1}{2} \ln \frac{\left(x_{1}-L\right)^{2}+x_{2}^{2}}{\left(x_{1}+L\right)^{2}+x_{2}^{2}}+\frac{\left(x_{1}-L\right)^{2}}{\left(x_{1}-L\right)^{2}+x_{2}^{2}}-\frac{\left(x_{1}+L\right)^{2}}{\left(x_{1}+L\right)^{2}+x_{2}^{2}}\right] \\
& \sigma_{33}^{D}=\frac{G \Omega_{3} \nu}{2 \pi(1-\nu)} \ln \frac{\left(x_{1}-L\right)^{2}+x_{2}^{2}}{\left(x_{1}+L\right)^{2}+x_{2}^{2}} \\
& \sigma_{12}^{D}=-\frac{G \Omega_{3} x_{2}}{2 \pi(1-\nu)}\left[\frac{x_{1}-L}{\left(x_{1}-L\right)^{2}+x_{2}^{2}}-\frac{x_{1}+L}{\left(x_{1}+L\right)^{2}+x_{2}^{2}}\right] \\
& \delta_{23}^{D}=0 \\
& \sigma_{31}^{D}=0
\end{aligned}
$$

These results agree with the stress at large distances from a finite dislocation wall found by Li [12], except for a $90^{\circ}$ rotation of the $x_{1} x_{2}$ plane.

Finally, to compare the finite with the infinitesimal dipole, we let the two basic disclinations approach each other. When $L \rightarrow 0$ in (9.5) the displacement of the finite dipole approaches

$$
\begin{aligned}
& u_{1}^{D} \rightarrow \frac{2 L \Omega_{1} x_{3}}{4 \pi(1-\nu)}\left[(1-2 \nu) \frac{x_{1}}{\rho^{2}}-2 \frac{x_{1} x_{2}^{2}}{\rho^{4}}\right]+\frac{2 L \Omega_{2} x_{3}}{4 \pi(1-\nu)}\left[(1-2 \nu) \frac{x_{2}}{\rho^{2}}+2 \frac{x_{1}^{2} x_{2}}{\rho^{4}}\right] \\
& -\frac{2 L \Omega_{3}}{4 \pi(1-\nu)}\left[(1-2 \nu) \ln \rho+\frac{x_{2}^{2}}{\rho^{2}}\right], \\
& u_{2}^{D} \rightarrow-\frac{2 L \Omega_{2} x_{3}}{4 \pi(1-\nu)}\left[(1-2 \nu) \frac{x_{2}}{\rho^{2}}-2 \frac{x_{1}^{2} x_{2}}{\rho^{4}}\right]+\frac{2 L \Omega_{2} x_{3}}{4 \pi(1-\nu)}\left[(1-2 \nu) \frac{x_{1}}{\rho^{2}}+2 \frac{x_{1} x_{2}^{2}}{\rho^{4}}\right] \\
& -2 L \Omega_{3}\left[\frac{\varphi}{2 \pi}-\frac{x_{1} x_{2}}{4 \pi(1-\nu) \rho^{2}}\right], \\
& u_{3}^{D} \rightarrow \frac{2 L \Omega_{1}}{4 \pi(1-\nu)}\left[(1-2 \nu) \ln \rho+\frac{x_{2}^{2}}{\rho^{2}}\right]+2 L \Omega_{3}\left[\frac{\varphi}{2 \pi}-\frac{x_{1} x_{2}}{4 \pi(1-\nu) \rho^{2}}\right] .
\end{aligned}
$$

These results agree with the displacement of the infinitesimal dipole (7.6) if we put $\xi_{r}=(2 L, 0,0)$. This was to be expected since this dipole is built up by confining the translation of the basic 
disclination to the $x_{1}$ axis. Next we let $L \rightarrow 0$ in (9.6) and find that the stress of the finite wedge dipole approaches

$$
\begin{aligned}
& \sigma_{11}^{D} \rightarrow-\frac{2 L G \Omega_{3}}{2 \pi(1-\nu)}\left[\frac{x_{1}}{\rho^{2}}-2 \frac{x_{1} x_{2}^{2}}{\rho^{4}}\right], \\
& \sigma_{22}^{D} \rightarrow-\frac{2 L G \Omega_{3}}{2 \pi(1-\nu)}\left[\frac{x_{1}}{\rho^{2}}+2 \frac{x_{1} x_{2}^{2}}{\rho^{4}}\right], \\
& \sigma_{33}^{D} \rightarrow-\frac{2 L G \Omega_{3} \nu}{2 \pi(1-\nu)} 2 \frac{x_{1}}{\rho^{2}}, \\
& \sigma_{12}^{D} \rightarrow \frac{2 L G \Omega_{3}}{2 \pi(1-\nu)}\left[\frac{x_{2}}{\rho^{2}}-2 \frac{x_{1}^{2} x_{2}}{\rho^{4}}\right] .
\end{aligned}
$$

These results should agree with the stress of the infinitesimal biaxial wedge disclination dipole, which we did not calculate in section 7 . However, we noted there that the fields of such a dipole are the same as those of an edge dislocation. If we substitute the translation found above, $\xi_{r}=(2 L$, $0,0)$, into (7.17) we find $b_{l}=\left(0,-2 L \Omega_{3}, 0\right)$. Now (9.8) agrees with (3.19) with this particular value for the Burgers vector.

\subsection{The Uniaxial Dipole}

We find the finite straight uniaxial disclination dipole by moving the axis of each basic disclination in section 9.1 back to the $x_{3}$ axis. We shall sketch out how to get results for this case. In general the axis of a discrete disclination line is translated from the point $x_{r}^{0}$ to the point $x_{r}^{0^{\prime}}$ by adding to it a discrete dislocation line whose dislocation density is given by, (II5.24),

$$
\alpha_{p l}(\mathbf{r})=\delta_{p}(L) \epsilon_{l q r} \Omega_{q}\left(x_{r}^{0}-x_{r}^{0^{\prime}}\right) .
$$

In our special case $x_{r}^{0^{\prime}}=0$, because we move the axis to the origin. For the positive disclination passing through $(L, 0,0)$ and the negative through $(-L, 0,0)$ parallel to the $x_{3}$ axis we find that the only nonvanishing components of $\delta_{p}(L)$ are

$$
\begin{aligned}
& \delta_{3}(L)=\delta\left(x_{1}-L\right) \delta\left(x_{2}\right), \text { at } x_{r}^{0}=(L, 0,0), \\
& \delta_{3}(L)=\delta\left(x_{1}+L\right) \delta\left(x_{2}\right), \text { at } x_{r}^{0}=(-L, 0,0) .
\end{aligned}
$$

by a coordinate translation of (A15). Hence, we find that the only nonvanishing components of the additional dislocations (9.9) are

$$
\begin{aligned}
& \alpha_{32}^{A}=\Omega_{3} L\left[\delta\left(x_{1}-L\right)+\delta\left(x_{1}+L\right)\right] \delta\left(x_{2}\right), \\
& \alpha_{33}^{A}=-\Omega_{2} L\left[\delta\left(x_{1}-L\right)+\delta\left(x_{1}+L\right)\right] \delta\left(x_{2}\right) .
\end{aligned}
$$

We now find the dislocation density from

$$
\alpha_{p l}^{L}=\alpha_{p l}^{D}+\alpha_{p l}^{A} .
$$

Note the change in sign between this relation and (7.12). Relation (9.12) gives the dislocation density of the finite uniaxial disclination dipole if $\alpha_{p l}^{D}$ is given by (9.1), but also of the corresponding dislocation model if $\alpha_{p l}^{D}$ is given by (9.3). The disclination density of the uniaxial dipole remains the 
same as for the biaxial dipole, (9.2) or (9.4). The additional dislocations (9.11) are also illustrated in figures $13-14$.

The static fields of the uniaxial dipole can be found simply by adding the fields of the dislocations (9.11) to the fields of the biaxial dipole in section 9.1. The procedure is straightforward and we leave the details to the reader.

\section{The Compensated Disclination Line}

As discussed in section III0 the compensated disclination line is obtained by eliminating the dislocation loop density, (II10.1),

$$
\beta_{k l}^{*}=0
$$

from the defect surface $S$ that spans the disclination line $L$. We shall illustrate this for our special case of a straight disclination. There are of course many possible choices for the surface $S$. We shall choose two of them, first the one already used and illustrated in figure 1 , and second the semi-infinite $x_{2} x_{3}$ plane for positive $x_{2}$.

From (10.1) and (4.5) we see that the plastic strain also vanishes, (II10.3),

$$
e_{k l}^{P}=0 \text {. }
$$

With the choice for $S$ as in figure 1 the disclination loop density remains the same as before, (4.4), and becomes the plastic bend-twist, (II10.4). So it has the only nonvanishing components

$$
\kappa_{2 q}^{P}=\varphi_{2 q}^{*}=\Omega_{q} H\left(-x_{1}\right) \delta\left(x_{2}\right)
$$

The defect densities of the compensated disclination are in general given by, (II10.5),

$$
\begin{aligned}
& \alpha_{p l}(\mathbf{r})=\delta_{p l} \delta_{k}(S) \Omega_{k}-\delta_{l}(S) \Omega_{p}, \\
& \theta_{p q}(\mathbf{r})=\delta_{p}(L) \Omega_{q} .
\end{aligned}
$$

For our special case we find the only nonvanishing components

$$
\begin{aligned}
& \alpha_{11}=-\Omega_{2} H\left(-x_{1}\right) \delta\left(x_{2}\right), \\
& \alpha_{12}=\Omega_{1} H\left(-x_{1}\right) \delta\left(x_{2}\right), \\
& \alpha_{32}=\Omega_{3} H\left(-x_{1}\right) \delta\left(x_{2}\right), \\
& \alpha_{33}=-\Omega_{2} H\left(-x_{1}\right) \delta\left(x_{2}\right), \\
& \theta_{3 q}=\Omega_{q} \delta\left(x_{1}\right) \delta\left(x_{2}\right),
\end{aligned}
$$

where we have used (A15-16). The compensated disclination consists of the same disclination line along $L$ as the corresponding discrete disclination, given by (4.12), and a constant dislocation distribution over the surface $S$, which is opposite to that of the dislocation model, given by (8.4). The latter distribution is a simple rotation wall, as we saw. The components $\alpha_{12}$ and $\alpha_{32}$ represent tilt walls, while the components $\alpha_{11}$ and $\alpha_{33}$ represent a twist wall.

For consistency it can be verified that (10.1-3) and (10.6-7) satisfy the definitions (4.13-14), as well as the relations (4.15-16). It is also easily verified that the continuity conditions (4.17-18) are satisfied by (10.6-7), in agreement with (II10.7-8). 
The incompatibility tensor is found by substituting (10.6-7) into (4.30) and we find

$$
\eta_{p q}=0
$$

In general we know that the total displacement and elastic strain vanish for the compensated disclination line, (II10.11-12),

$$
\begin{gathered}
u_{n}^{T}=0, \\
e_{k l}=0 .
\end{gathered}
$$

The last result (10.10) is consistent with (10.8), because the incompatibility is the source of elastic strain. Since the elastic strain vanishes we see by Hookes' law that the stress also vanishes. The only elastic field that does not vanish is the elastic bend-twist. It is in general given by, (II10.13),

$$
\kappa_{k q}=-\kappa_{k q}^{P},
$$

and for our special case has the only non-vanishing components

$$
\kappa_{2 q}=-\Omega_{q} H\left(-x_{1}\right) \delta\left(x_{2}\right),
$$

by (10.3).

We note that if we add the results of the compensated disclination to those of the dislocation model in section 8 we get the results for the disclination line in sections $4-5$, as we already pointed out for the general case in section II10. For example, we have, symbolically, $(10.1)+(8.2)=(4.3)$, $(10.3)+(8.1)=(4.4),(10.6)+(8.4)=(4.11),(10.7)+(8.5)=(4.12),(10.8)+(8.6)=(4.31)$, and $(10.12)+(8.10)=(5.9)$.

We now investigate the other choice for the surface $S$, namely the semi-infinite plane lying in the $x_{2} x_{3}$ plane for positive $x_{2}$ and with a normal in the negative $x_{1}$ direction. This geometry can easily be obtained by rotating the plane $S$ of figure 1 clockwise by $90^{\circ}$. Then the only nonvanishing component of $\delta_{p}(S)$ becomes

$$
\delta_{1}(S)=-\delta\left(x_{1}\right) H\left(x_{2}\right)
$$

instead of (A16). Hence from (4.2) the only nonvanishing components of the plastic bend-twist and disclination loop densities become

$$
\kappa_{1 q}^{P}=\varphi_{1 q}^{*}=\Omega_{q} \delta\left(x_{1}\right) H\left(x_{2}\right),
$$

instead of (10.3). Furthermore from (10.4) the only nonvanishing components of the dislocation density now become

$$
\begin{aligned}
& \alpha_{21}=\Omega_{2} \delta\left(x_{1}\right) H\left(x_{2}\right), \\
& \alpha_{31}=\Omega_{3} \delta\left(x_{1}\right) H\left(x_{2}\right), \\
& \alpha_{22}=-\Omega_{1} \delta\left(x_{1}\right) H\left(x_{2}\right), \\
& \alpha_{33}=-\Omega_{1} \delta\left(x_{1}\right) H\left(x_{2}\right),
\end{aligned}
$$

instead of (10.6). Finally the elastic bend-twist is also changed according to (10.11) and (10.14). All other relations remain the same for either choice of the surface $S$. 


\section{The Bent Dislocation Wall}

In section 10 we found the fields of the compensated disclination for two different choices of the defect surface $S$. If we take the difference between the results for these two geometries all the fields vanish, except the plastic and elastic bend-twist, the disclination loop density, and the dislocation density. The latter becomes from (10.6) and (10.15)

$$
\begin{aligned}
& \alpha_{11}=\Omega_{2} H\left(-x_{1}\right) \delta\left(x_{2}\right), \\
& \alpha_{21}=\Omega_{2} \delta\left(x_{1}\right) H\left(x_{2}\right), \\
& \alpha_{31}=\Omega_{3} \delta\left(x_{1}\right) H\left(x_{2}\right), \\
& \alpha_{12}=-\Omega_{1} H\left(-x_{1}\right) \delta\left(x_{2}\right), \\
& \alpha_{22}=-\Omega_{1} \delta\left(x_{1}\right) H\left(x_{2}\right), \\
& \alpha_{32}=-\Omega_{3} H\left(-x_{1}\right) \delta\left(x_{2}\right), \\
& \alpha_{13}=0, \\
& \alpha_{23}=0, \\
& \alpha_{33}=-\Omega_{1} \delta\left(x_{1}\right) H\left(x_{2}\right)+\Omega_{2} \mathrm{H}\left(-x_{1}\right) \delta\left(x_{2}\right) .
\end{aligned}
$$

What kind of defect is this? It represents two semi-infinite dislocation walls that meet at the $x_{3}$ axis, i.e., it is bent dislocation wall. It has no long range stress or elastic strain. The three different cases are illustrated in figures 15-17, where for clarity we have shown the discrete rather than the constant dislocation distribution. Figures 15-16 show the geometry that has been observed by Schober and Baluffi [13], their figures 6 and 7.

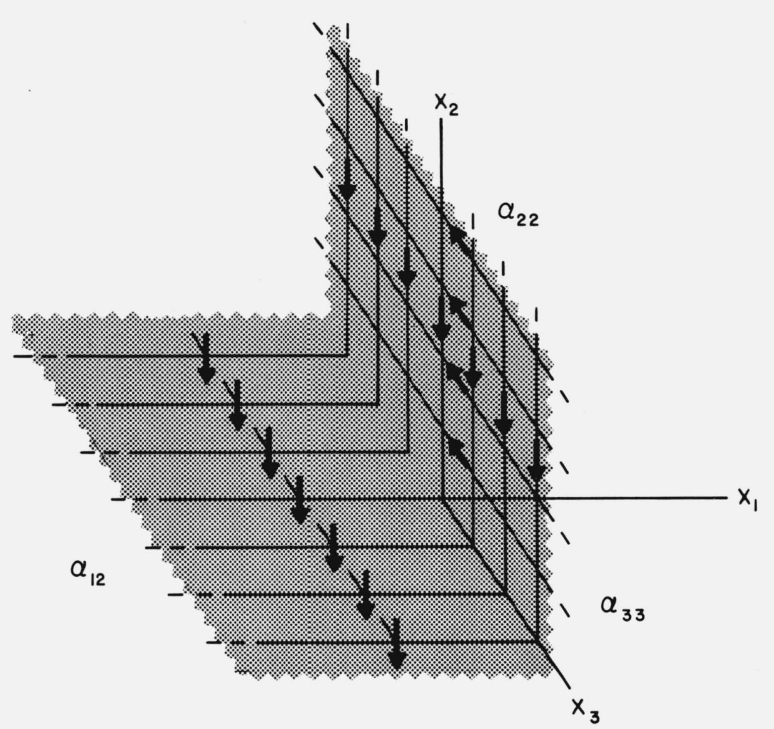

FIGURE 15. The bent dislocation wall with $\Omega_{1}$ rotation, described by the dislocation density $\alpha_{12}=-\Omega_{1} H\left(-x_{1}\right)$ $\delta\left(x_{2}\right)$ and $\alpha_{22}=\alpha_{33}=-\Omega_{1} \delta\left(x_{1}\right) H\left(x_{2}\right)$.

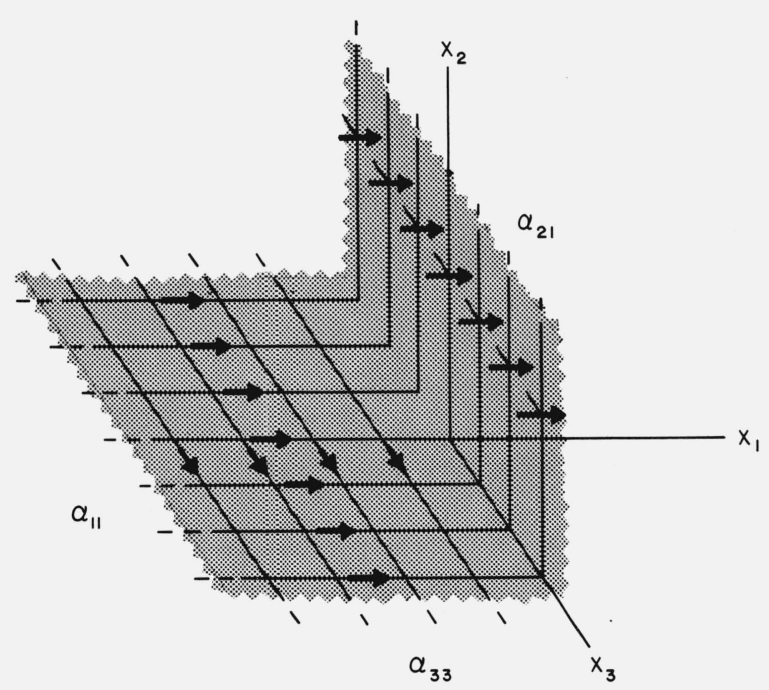

FIGURE 16. The bent dislocation wall with $\Omega_{2}$ rotation, described by the dislocation density $\alpha_{11}=\alpha_{33}=\Omega_{2} H\left(-x_{1}\right)$ $\delta\left(x_{2}\right)$ and $\alpha_{21}=\Omega_{2} \delta\left(x_{1}\right) H\left(x_{2}\right)$. 


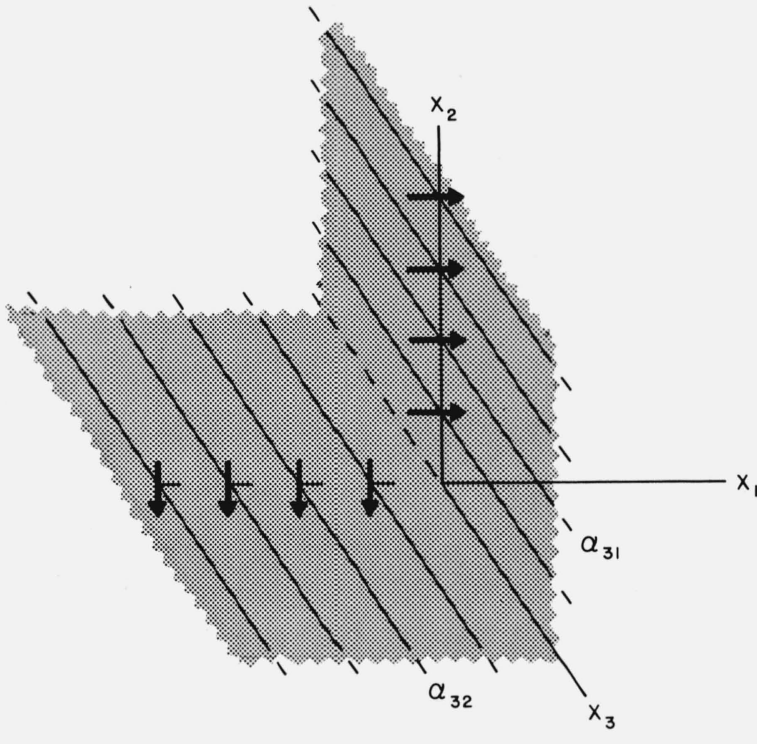

FIGURE 17. The bent dislocation wall with $\Omega_{3}$ rotation, described by the dislocation density $\alpha_{31}=\Omega_{3} \delta\left(x_{1}\right) H\left(x_{2}\right)$ and $\alpha_{32}=-\Omega_{3} H\left(-x_{1}\right) \delta\left(x_{2}\right)$.

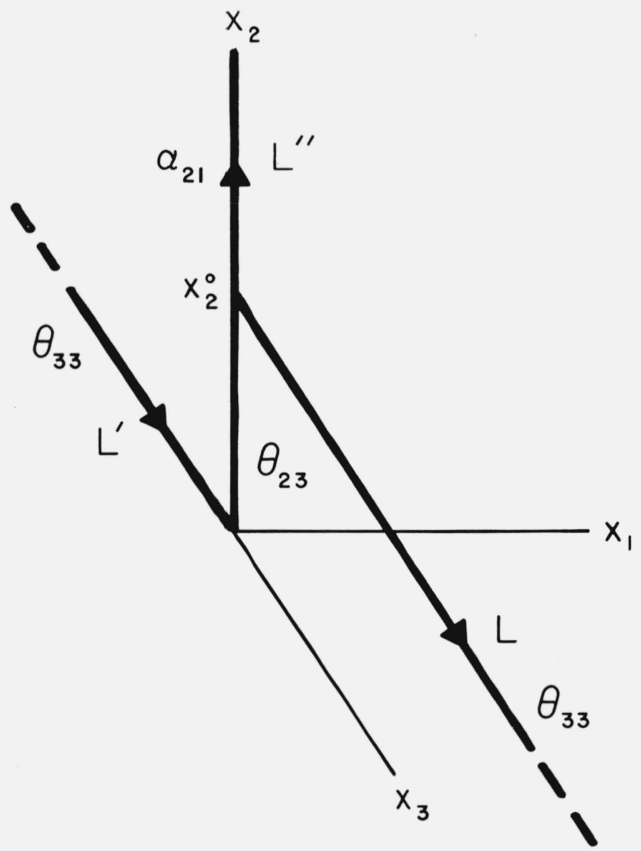

FIGURE 18. Edge dislocation terminating at a jog on a wedge disclination.

\section{Example of a Terminating Dislocation}

In a recent publication [14] we gave a concrete example of a discrete edge dislocation terminating on a jog in a discrete wedge disclination. We claimed that example illustrated the continuity equation (4.17). In this section we give a detailed analysis of the geometry of this example. Then we can verify the continuity equation for this case explicitly.

We have already derived the general expressions for a discrete dislocation terminating on a discrete disclination. The defect densities are given by, (II5.25-26),

$$
\begin{aligned}
& \alpha_{p l}(\mathbf{r})=\delta_{p}(L)\left\{b_{l}+\epsilon_{l q r} \Omega_{q}\left(x_{r}-x_{r}^{0}\right)\right\}+\delta_{p}\left(L^{\prime}\right)\left\{b_{l}+\epsilon_{l q r} \Omega_{q}\left(x_{r}-x_{r}^{0^{\prime}}\right)\right\}+\delta_{p}\left(L^{\prime \prime}\right) \boldsymbol{\epsilon}_{l q r} \Omega_{q}\left(x_{r}^{0}-x_{r}^{0^{\prime}} .\right), \\
& \theta_{p q}(\mathbf{r})=\delta_{p}\left(L+L^{\prime}\right) \Omega_{q} .
\end{aligned}
$$

Here the disclination line lies along the closed curve $L$ and $L^{\prime}$, and the dislocation line lies along the connecting link $L^{\prime \prime}$.

We choose the special case illustrated in figure 18. We set $b_{l}=x_{r}^{0^{\prime}} .=0$, and for $\Omega_{q}$ and $x_{r}^{0}$ we choose the only nonvanishing components $\Omega_{3}$ and $x_{2}^{0}>0$. Furthermore we choose for $L$ a line parallel to the positive $x_{3}$ axis passing through the point $\left(0, x_{2}^{0}, 0\right)$, for $L^{\prime}$ the negative $x_{3}$ axis and the small jog on the positive $x_{2}$ axis, running from the origin to $x_{2}^{0}$, and for $L^{\prime \prime}$ the rest of the positive $x_{2}$ axis beyond $x_{2}^{\circ}$. Then the only nonvanishing components of the delta functions in (12.1-2) are

$$
\begin{aligned}
& \delta_{3}(L)=\delta\left(x_{1}\right) \delta\left(x_{2}-x_{2}^{0}\right) H\left(x_{3}\right), \\
& \delta_{2}\left(L^{\prime}\right)=\delta\left(x_{1}\right) H\left(x_{2}\right) H\left(x_{2}^{0}-x_{2}\right) \delta\left(x_{3}\right), \\
& \delta_{3}\left(L^{\prime}\right)=\delta\left(x_{1}\right) \delta\left(x_{2}\right) H\left(-x_{3}\right), \\
& \delta_{2}\left(L^{\prime \prime}\right)=\delta\left(x_{1}\right) H\left(x_{2}-x_{2}^{0}\right) \delta\left(x_{3}\right) .
\end{aligned}
$$


Therefore we find for (12.1-2) the only nonvanishing components

$$
\begin{aligned}
& \alpha_{21}=-\Omega_{3} x_{2} \delta\left(x_{1}\right) H\left(x_{2}\right) H\left(x_{2}^{0}-x_{2}\right) \delta\left(x_{3}\right)-\Omega_{3} x_{2}^{0} \delta\left(x_{1}\right) H\left(x_{2}-x_{2}^{0}\right) \delta\left(x_{3}\right), \\
& \theta_{23}=\Omega_{3} \delta\left(x_{1}\right) H\left(x_{2}\right) H\left(x_{2}^{0}-x_{2}\right) \delta\left(x_{3}\right), \\
& \theta_{33}=\Omega_{3} \delta\left(x_{1}\right) \delta\left(x_{2}-x_{2}^{0}\right) H\left(x_{3}\right)+\Omega_{3} \delta\left(x_{1}\right) \delta\left(x_{2}\right) H\left(-x_{3}\right) .
\end{aligned}
$$

where we have used (A8). These relations represent a wedge disclination with a jog near the origin from which an edge dislocation emerges. Specifically, the disclination consists of a straight wedge disclination approaching the origin along the negative $x_{3}$ axis, (the second term in (12.5b)), a small jog of twist disclination running along the $x_{2}$ axis from the origin to $x_{2}^{0},(12.5 \mathrm{a})$, and a straight wedge disclination running parallel to the positive $x_{3}$ axis from the point $x_{2}^{0}$ on the $x_{2}$ axis, (the first term of (12.5b)). The dislocation consists of the following: The first term of (12.4) represents a dislocation that grows linearly in strength along the $x_{2}$ axis from the origin to the point $x_{2}^{0}$, and can be regarded as an integral part of the element of discrete twist disclination. The second term of (12.4) is an edge dislocation running along the positive $x_{2}$ axis starting from the point $x_{2}^{0}$. From (12.4) this edge dislocation has the Burgers vector

$$
b_{1}=-\Omega_{3} x_{2}^{0}
$$

Note that the effect of the terminating dislocation is to move the disclination as well as its axis from $x_{2}=0$ for $L^{\prime}$ to $x_{2}=x_{2}^{0}$ for $L$.

Now we shall show that $(12.4-5)$ satisfy the continuity equations $(4.17-18)$

$$
\begin{gathered}
\alpha_{p l, p}+\epsilon_{l p q} \theta_{p q}=0, \\
\theta_{p q, p}=0 .
\end{gathered}
$$

We need only consider the cases $l=1$ in (12.7) and $q=3$ in (12.8), for all other cases are identically satisfied. We have for (12.7)

$$
\begin{aligned}
\alpha_{21,2}+\theta_{23}=-\Omega_{3} \delta\left(x_{1}\right) H\left(x_{2}\right) H\left(x_{2}^{0}-x_{2}\right) \delta\left(x_{3}\right)+\Omega_{3} x_{2} \delta\left(x_{1}\right) H\left(x_{2}\right) \delta\left(x_{2}^{0}-x_{2}\right) \delta\left(x_{3}\right) \\
-\Omega_{3} x_{2}^{0} \delta\left(x_{1}\right) \delta\left(x_{2}-x_{2}^{0}\right) \delta\left(x_{3}\right)+\Omega_{3} \delta\left(x_{1}\right) H\left(x_{2}\right) H\left(x_{2}^{0}-x_{2}\right) \delta\left(x_{3}\right)
\end{aligned}
$$

$$
=0
$$

by $\left(\mathrm{A6} 6^{-7)}\right.$ and the assumption that $x_{2}^{0}>0$. We have for (12.8)

$$
\begin{aligned}
\theta_{23,2}+\theta_{33,3} & =\Omega_{3} \delta\left(x_{1}\right) \delta\left(x_{2}\right) H\left({ }_{2}^{0}-x_{2}\right) \delta\left(x_{3}\right) \\
& -\Omega_{3} \delta\left(x_{1}\right) H\left(x_{2}\right) \delta\left(x_{2}^{0}-x_{2}\right) \delta\left(x_{3}\right) \\
& +\Omega_{3} \delta\left(x_{1}\right) \delta\left(x_{2}-x_{2}^{0}\right) \delta\left(x_{3}\right)-\Omega_{3} \delta\left(x_{1}\right) \delta\left(x_{2}\right) \delta\left(x_{3}\right) \\
& =0
\end{aligned}
$$

similarly. So we have shown here by a detailed analysis that a dislocation can terminate on a disclination, and that this is consistent with the continuity equations. 


\section{Summary}

We have specialized results derived for the general theory to the special case of straight disclination lines. First we recalled the work of Volterra and reviewed the straight dislocation to introduce the subject.

We derived the geometry of straight disclination lines. We found the defect loop densities which are the same as Mura's "plastic distortion" and "plastic rotation." They are concentrated on the defect surface, which is a semi-infinite plane terminating at the disclination line. Then we identified the basic plastic fields (plastic strain and bend-twist) which are also concentrated on the defect surface. We found the defect densities (dislocation and disclination) which are singular along the disclination line. We noted that there is a finite dislocation density for the discrete disclination line, and we gave it an intuitively reasonable interpretation. Our results agree completely with Anthony's. We checked the consistency of our specific results by showing that they satisfy the necessary general equations: the definitions of the defect densities in terms of the basic plastic fields, the relations between defect loops and densities, and the continuity conditions. We illustrated the significance of the total Burgers vector as the displacement jump at the origin by showing that it vanishes for our special choice, whereas the displacement jump condition gives a result that is intuitively clear from the illustrations. The incompatibility tensor was found and shown to satisfy its continuity condition.

Then we found the isotropic static results for straight disclinations. The displacements of a straight disclination were written down. Our point of departure was to regard these expressions as an educated guess. By differentiating we found the total distortion. From this total distortion the basic total fields (strain and bend-twist) can be derived. They consist of two types of terms: the first terms are singular on the defect surface and correspond exactly to the basic plastic fields derived independently above and the second terms are continuous away from the singular disclination line. This then provides a natural separation of the basic fields into elastic and plastic parts. In this manner we found the elastic strain and bend-twist of a straight disclination line. We showed that they satisfied the field equations with the defect densities. The stress followed from Hooke's law. It satisfied the equilibrium equations, including at the singular defect line, thus confirming that the original displacements were correct.

Then we showed how the fields were modified when the disclination axis was moved away from the origin. The dislocation density and total Burgers vector were changed whereas the disclination density and Frank vector remained the same. The results can be interpreted by saying that the axis is moved by adding a dislocation to the disclination line. This observation facilitates finding the static fields of the disclination with the new axis. The fields are found by adding those for a dislocation to those for a disclination with its axis thru the origin.

We derived results for the infinitesimal disclination dipole, which can be biaxial or uniaxial. We found the defect densities and displacements of both types and the relations between them. We noted that the displacement of the straight uniaxial dipole does not contain a wedge component. Therefore the static fields of the straight biaxial wedge disclination dipole are the same as those of a straight edge dislocation, confirming a result published by Eshelby.

The dislocation model of a straight disclination was found to be a terminating dislocation wall (tilt and twist). We found that the dislocation model and its corresponding disclination line have the same incompatibility tensor, displacement, total distortion, elastic strain, and stress. The total distortion contains terms that are singular on the defect surface, which correspond exactly to the dislocation loop density derived independently before. If these terms are identified as the plastic distortion, as Mura did, the dislocation model follows. In this way we found the interpretation of Mura's "elastic distortion" of the discrete disclination as the elastic distortion of the corresponding dislocation model. Finally we interpreted Mura's "plastic rotation" as the difference in bend-twist between the disclination and its dislocation model.

Next we found the defect densities of the finite diselination dipole. It was also possible to construct the dislocation model for this defect. The displacement of the biaxial dipole was found. It 
approaches the displacement of the infinitesimal dipole when the width of the dipole approaches zero. The stress of the biaxial wedge disclination dipole was found to correspond to an earlier calculation of $\mathrm{Li}$ for the finite dislocation wall.

We found the defect densities for the compensated disclination line, using two different defect surfaces. From these results we constructed the bent dislocation wall, which has been observed by Schober and Baluffi. These defects have no displacement, elastic strain, or stress.

Finally we showed in analytical detail how a discrete edge dislocation can end on a jog in a discrete wedge disclination. The jog is a small segment of twist disclination line, which contains a finite amount of dislocation density. We showed that the analytic results satisfy the continuity conditions.

In appendix A we show how the general Dirac delta functions for a curve and a surface, which are defined for and occur in the general theory, are specialized to the case of a straight line and a semi-infinite plane. It is essential to know this type of specialization in order to apply the general theory to particular geometries. We also calculate some useful Burgers circuit integrals.

In appendix B we show how to take the generalized derivatives of the cylindrical coordinates. We carefully define the cylindrical angle $\varphi$ in terms of the Cartesian Coordinates $x_{1}$ and $x_{2}$, restricting it to the range $(-\pi, \pi)$. Its derivative is regarded as a generalized derivative and contains a delta function. Also functions whose degree of homogeneity is -1 or less will have generalized functions in their generalized derivatives. The usefulness of these generalized function techniques is that they allow us to carry through the traditional field operations, such as differentiation, even at singular lines and surfaces, in the field theoretic framework that is traditionally set up for continuous well-behaved functions only. This simplifies the work for discrete defects considerably, since we can apply straightforward mathematical techniques at the singularities, instead of intuitive notions as is done classically.

In appendix $\mathrm{C}$ we show how the isotropic general results of III can be used to find the functional dependence of the static fields for straight discrete defects.

\section{Appendix A. Evaluation of the Delta Function on a Straight Line and a Semi-Infinite Plane}

The Dirac delta function $\delta(x)$ is defined by, (IIBl),

$$
\int_{a}^{b} \delta\left(x-x^{\prime}\right) \varphi\left(x^{\prime}\right) d x^{\prime}= \begin{cases}0, & \text { if } x<a \\ \varphi(x), & \text { if } a<x<b, \\ 0, & \text { if } b<x .\end{cases}
$$

This relation is illustrated by the following special cases

$$
\begin{gathered}
\int_{-\infty}^{\infty} \delta\left(x-x^{\prime}\right) d x^{\prime}=1, \\
\int_{0}^{\infty} \delta\left(x-x^{\prime}\right) d x^{\prime}=H(x), \\
\int_{-\infty}^{0} \delta\left(x-x^{\prime}\right) d x^{\prime}=H(-x),
\end{gathered}
$$

where we have introduced the Heaviside function defined by

$$
H(x) \equiv \begin{cases}0, & \text { if } x<0 \\ 1, & \text { if } 0<x\end{cases}
$$

The derivative of the Heaviside function is easily found from (A3) by a partial integration 


$$
\begin{aligned}
H^{\prime}(x) & =\frac{\partial H(x)}{\partial x} \\
& =\int_{0}^{\infty} \frac{\partial}{\partial x} \delta\left(x-x^{\prime}\right) d x^{\prime} \\
& =-\int_{0}^{\infty} \frac{\partial}{\partial x^{\prime}} \delta\left(x-x^{\prime}\right) d x^{\prime} \\
& =-\left[\delta\left(x-x^{\prime}\right)\right]_{0}^{\infty} \\
& =\delta(x),
\end{aligned}
$$

since $\delta(x)=0$ whenever $x \neq 0$. From (Al) we see that we also have

$$
\delta\left(x-x^{\prime}\right) \varphi\left(x^{\prime}\right)=\delta\left(x-x^{\prime}\right) \varphi(x)
$$

A particular case of this relation is

$$
\left(x-x^{\prime}\right) \delta\left(x-x^{\prime}\right)=0 .
$$

By differentiating this relation we find

$$
\left(x-x^{\prime}\right) \delta^{\prime}\left(x-x^{\prime}\right)=-\delta\left(x-x^{\prime}\right)
$$

The three-dimensional Dirac delta function $\delta(\mathbf{r})$ is defined by, (IIB2),

$$
\delta\left(\mathbf{r}-\mathbf{r}^{\prime}\right) \equiv \delta\left(x_{1}-x_{1}^{\prime}\right) \delta\left(x_{2}-x_{2}^{\prime}\right) \delta\left(x_{3}-x_{3}^{\prime}\right) .
$$

The Dirac delta functions for a curve $L$ and a surface $S$ are defined by, (IIB4-5),

$$
\begin{aligned}
& \delta_{i}(L) \equiv \int_{L} \delta\left(\mathbf{r}-\mathbf{r}^{\prime}\right) d L_{i}^{\prime}, \\
& \delta_{i}(S) \equiv \int_{S} \delta\left(\mathbf{r}-\mathbf{r}^{\prime}\right) d S_{i}^{\prime} .
\end{aligned}
$$

We want to evaluate these quantities for the special case when $L$ is the straight line lying along the $x_{3}$ axis and $S$ is the semi-infinite plane lying in the $x_{1} x_{3}$ plane for negative $x_{1}$ and with a normal in the negative $x_{2}$ direction, as illustrated in figure 1 . Then we have in particular for $L$

$$
\begin{aligned}
d L_{i}^{\prime} & =\left(0,0, d L_{3}^{\prime}\right)=\left(0,0, d x_{3}^{\prime}\right), \\
\mathbf{r}^{\prime} & =\left(0,0, x_{3}^{\prime}\right),
\end{aligned}
$$

and for $S$

$$
\begin{aligned}
d S_{i}^{\prime} & =\left(0, d S_{2}^{\prime}, 0\right)=\left(0,-d x_{1}^{\prime} d x_{3}^{\prime}, 0\right), \\
\mathbf{r}^{\prime} & =\left(x_{1}^{\prime}, 0, x_{3}^{\prime}\right) .
\end{aligned}
$$

We find that the only nonvanishing components of (A11-12) are

$$
\delta_{3}(L)=\int_{-\infty}^{\infty} \delta\left(x_{1}\right) \delta\left(x_{2}\right) \delta\left(x_{3}-x_{3}^{\prime}\right) d x_{3}^{\prime}
$$




$$
\begin{aligned}
& =\delta\left(x_{1}\right) \delta\left(x_{2}\right) \\
\delta_{2}(S) & =-\int_{-\infty}^{\infty} \int_{-\infty}^{0} \delta\left(x_{1}-x_{1}^{\prime}\right) \delta\left(x_{2}\right) \delta\left(x_{3}-x_{3}^{\prime}\right) d x_{1}^{\prime} d x_{3}^{\prime} \\
& =-H\left(-x_{1}\right) \delta\left(x_{2}\right),
\end{aligned}
$$

where we have used (A2) and (A4). We note that these results satisfy Stokes' theorem, (IIB26),

$$
\epsilon_{i j k} \delta_{i, j}(S)=-\delta_{k}(L)
$$

by (A6). This was to be expected since $L$ is the boundary of $S$. Next we want to consider some integrals along a curve $\lambda$ that links $L$. We take the special case where $\lambda$ is a circle of radius $a$ in the $x_{1} x_{2}$ plane centered at the origin, also illustrated in figure 1 . Then we have

$$
x_{1}^{2}+x_{2}^{2}=a^{2}
$$

on $\lambda$. We wish to evaluate three typical closed line integrals on $\lambda$ involving generalized functions. The first one is

$$
\begin{aligned}
\oint_{\lambda} f\left(x_{1}\right) \delta\left(x_{2}\right) d L_{1} & =-\oint_{\lambda} f\left(x_{1}\right) \delta\left(x_{2}\right) x_{2}\left(a^{2}-x_{2}^{2}\right)^{-1 / 2} d L_{2} \\
& =0
\end{aligned}
$$

where we have used (A18) to change the variable of integration, and (A8). The second one is

$$
\begin{aligned}
\oint_{\lambda} f\left(x_{1}\right) \delta\left(x_{2}\right) d L_{2} & =\int_{-a}^{a} f\left(\left[a^{2}-x_{2}^{2}\right]^{1 / 2}\right) \delta\left(x_{2}\right) d x_{2}+\int_{a}^{-a} f\left(-\left[a^{2}-x_{2}^{2}\right]^{1 / 2}\right) \delta\left(x_{2}\right) d x_{2} \\
& =f(a)-f(-a),
\end{aligned}
$$

where we have used (A18) and (A1). The third one is

$$
\begin{aligned}
\oint_{\lambda} f\left(x_{1}\right) \delta^{\prime}\left(x_{2}\right) d L_{2} & =-\oint_{\lambda} f^{\prime}\left(x_{1}\right) \delta\left(x_{2}\right) d L_{1} \\
& =0
\end{aligned}
$$

by a partial integration and (A19). Finally, for the sake of consistency we wish to show that the results (A15-16) satisfy (IIB15). Let $\sigma$ be the circular plane bounded by $\lambda$ (fig. 1). Then we have from (A15)

$$
\begin{aligned}
\int_{\sigma} \delta_{3}(L) d S_{3} & =\iint \delta\left(x_{1}\right) \delta\left(x_{2}\right) d x_{1} d x_{2} \\
& =1,
\end{aligned}
$$

by (A1). Next we have from (A16)

$$
\begin{aligned}
\oint_{\lambda} \delta_{2}(S) d L_{2} & =-\oint_{\lambda} H\left(-x_{1}\right) \delta\left(x_{2}\right) d L_{2} \\
& =-H(-a)+H(a) \\
& =1
\end{aligned}
$$

by (A20) and (A5). So these results agree with (IIB15). 


\section{Appendix B. Generalized Derivatives of Cylindrical Coordinates}

The expressions that are differentiated in the text contain the cylindrical coordinates $\rho$ and $\varphi$. The geometric relation of these coordinates to the Cartesian Coordinates $x_{1}$ and $x_{2}$ is illustrated in figure 5. The functional relation for $\rho$ is

$$
\rho\left(x_{1}, x_{2}\right)=\left(x_{1}^{2}+x_{2}^{2}\right)^{1 / 2} \text {. }
$$

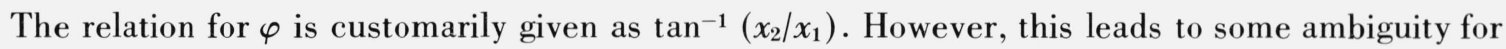
two reasons: (1) The arctangent is usually taken to be restricted to its principal value, lying in the range $(-\pi / 2, \pi / 2)$, especially in integral formulas. (2) The angle $\varphi$ is usually regarded to be multiplevalued, a procedure that conflicts with the assumption of uniqueness for the functions that occur in the general defect theory. Therefore we shall arbitrarily restrict $\varphi$ to the range $(-\pi, \pi)$, so that it is uniquely defined everywhere, but possesses a discontinuity of $2 \pi$ across the negative $x_{1}$ axis, as shown in figure 5 . This choice is convenient for the calculations in the text, for then the discontinuity occurs at the semi-infinite plane $S$ of appendix A (fig. 1). So the functional relation for $\varphi$ is

$$
\varphi\left(x_{1}, x_{2}\right)=\tan ^{-1}\left(x_{2} / x_{1}\right)+\pi H\left(-x_{1}\right)\left[H\left(x_{2}\right)-H\left(-x_{2}\right)\right],
$$

where the arctangent is restricted to its principal value and the Heaviside function $H$ is defined by (A5). We see that $\varphi$, so defined, assumes the values indicated in figure 5 .

In order to maintain the necessary uniqueness when differentiating the discontinuous function $\varphi$, it becomes necessary to take the generalized derivative. The discontinuity will then lead to a generalized function, the Dirac delta function. We refer to Gel'fand and Shilov [8], chapter 1, section 2.2 , for the detailed procedure on taking this generalized derivative. The result is

$$
\begin{aligned}
& \varphi_{, 1}=-x_{2} / \rho^{2}, \\
& \varphi_{, 2}=x_{1} / \rho^{2}+2 \pi H\left(-x_{1}\right) \delta\left(x_{2}\right) .
\end{aligned}
$$

Intuitively, these results could have been anticipated. They contain the ordinary derivatives of the arctangent function plus a term having a delta function across the negative $x_{1}$ axis, which originates from the discontinuity of $\varphi$.

By contrast the generalized derivatives of $\rho$ and $\ln \rho$ are the same as the straightforward classical ones. For the latter we have

$$
\begin{aligned}
& (\ln \rho)_{, 1}=x_{1} / \rho^{2}, \\
& (\ln \rho)_{, 2}=x_{2} / \rho^{2} .
\end{aligned}
$$

Another function whose generalized and classical derivatives coincide is the following

$$
\left(\frac{x_{\alpha} x_{\beta}}{\rho^{2}}\right)_{, \gamma}=-2 \frac{x_{\alpha} x_{\beta} x_{\gamma}}{\rho^{4}}+\frac{x_{\alpha} \delta_{\beta \gamma}+x_{\beta} \delta \gamma_{\alpha}}{\rho^{2}},(\alpha, \beta, \gamma=1,2)
$$

where we have used the convention that Greek subscripts have the range 1,2. When written out in full these equations are

$$
\begin{aligned}
\left(\frac{x_{1}^{2}}{\rho^{2}}\right)_{, 1} & =2 \frac{x_{1} x_{2}^{2}}{\rho^{4}}, & \left(\frac{x_{1}^{2}}{\rho^{2}}\right)_{, 2} & =-2 \frac{x_{1}^{2} x_{2}}{\rho^{4}}, \\
\left(\frac{x_{1} x_{2}}{\rho^{2}}\right)_{, 1} & =-2 \frac{x_{1}^{2} x_{2}}{\rho^{4}}+\frac{x_{2}}{\rho^{2}}, & \left(\frac{x_{1} x_{2}}{\rho^{2}}\right)_{, 2} & =-2 \frac{x_{1} x_{2}^{2}}{\rho^{4}}+\frac{x_{1}}{\rho^{2}},
\end{aligned}
$$




$$
\left(\frac{x_{2}^{2}}{\rho^{2}}\right)_{, 1}=-2 \frac{x_{1} x_{2}^{2}}{\rho^{4}}, \quad\left(\frac{x_{2}^{2}}{\rho^{2}}\right)_{, 2}=2 \frac{x_{1}^{2} x_{2}}{\rho^{4}} .
$$

The above derivatives (B5-7) are all locally integrable in the $x_{1} x_{2}$ plane, including the singularity at the origin. This is the reason why the generalized and classical functions coincide.

However, if we take further derivatives of (B7), the results (having a $\rho^{-2}$ dependence) will no longer be locally integrable at the origin, because then the algebraic singularity has the same strength as the dimension of the space (i.e., 2). To maintain uniqueness it becomes again necessary to take the generalized derivative. We refer also to Gel'fand and Shilov [8], chapter III, section 3.3, for the detailed procedure on taking this type of generalized derivative. The results will contain expressions homogeneous of degree -2 (such as $\rho^{-2}$ ), which have to be regarded as generalized functions. One result is

$$
\left(\frac{x_{\alpha}}{\rho^{2}}\right)_{, \beta}=\frac{\delta_{\alpha \beta}}{\rho^{2}}-2 \frac{x_{\alpha} x_{\beta}}{\rho^{4}}+\pi \delta_{\alpha \beta} \delta(\boldsymbol{\rho})
$$

where we have defined the two-dimensional Dirac delta function

$$
\delta(\boldsymbol{\rho})=\delta\left(x_{1}\right) \delta\left(x_{2}\right)
$$

When (B8) is written out in full we have

$$
\begin{array}{ll}
\left(\frac{x_{1}}{\rho^{2}}\right)_{, 1}=\frac{1}{\rho^{2}}-2 \frac{x_{1}^{2}}{\rho^{4}}+\pi \delta(\boldsymbol{\rho}), & \left(\frac{x_{1}}{\rho^{2}}\right)_{, 2}=-2 \frac{x_{1} x_{2}}{\rho^{4}}, \\
\left(\frac{x_{2}}{\rho^{2}}\right)_{, 1}=-2 \frac{x_{1} x_{2}}{\rho^{4}}, & \left(\frac{x_{2}}{\rho^{2}}\right)_{, 2}=\frac{1}{\rho^{2}}-2 \frac{x_{2}^{2}}{\rho^{4}}+\pi \delta(\boldsymbol{\rho}) .
\end{array}
$$

Another result is

$$
\left(\frac{x_{\alpha} x_{\beta} x_{\gamma}}{\rho^{4}}\right)_{, \delta}=-4 \frac{x_{\alpha} x_{\beta} x \gamma x_{\delta}}{\rho^{6}}+\frac{x_{\alpha} x_{\beta} \delta \gamma_{\delta}+x_{\alpha} \delta_{\beta \delta} x \gamma+\delta_{\alpha \delta} x_{\beta} x \gamma}{\rho^{4}}+\frac{\pi}{4}\left(\delta_{\alpha \beta} \delta \gamma_{\delta}+\delta \gamma_{\alpha} \delta_{\beta \delta}+\delta_{\beta \gamma} \delta_{\alpha \delta}\right) \delta(\boldsymbol{\rho}),
$$

or, when written out in full

$$
\begin{array}{rlrl}
\left(\frac{x_{1}^{3}}{\rho^{4}}\right)_{, 1} & =-4 \frac{x_{1}^{4}}{\rho^{6}}+3 \frac{x_{1}^{2}}{\rho^{4}}+\frac{3 \pi}{4} \delta(\rho), & \left(\frac{x_{1}^{3}}{\rho^{4}}\right)_{, 2}=-4 \frac{x_{1}^{3} x_{2}}{\rho^{6}}, \\
\left(\frac{x_{1}^{2} x_{2}}{\rho^{4}}\right)_{, 1}=-4 \frac{x_{1}^{3} x_{2}}{\rho^{6}}+2 \frac{x_{1} x_{2}}{\rho^{4}}, & \left(\frac{x_{1}^{2} x_{2}}{\rho^{4}}\right)_{, 2}=-4 \frac{x_{1}^{2} x_{2}^{2}}{\rho^{6}}+\frac{x_{1}^{2}}{\rho^{4}}+\frac{\pi}{4} \delta(\rho), \\
\left(\frac{x_{1} x_{2}^{2}}{\rho^{4}}\right)_{, 1}=-4 \frac{x_{1}^{2} x_{2}^{2}}{\rho^{6}}+\frac{x_{2}^{2}}{\rho^{4}}+\frac{\pi}{4} \delta(\rho), & \left(\frac{x_{1} x_{2}^{2}}{\rho^{4}}\right)_{, 2}=-4 \frac{x_{1} x_{2}^{3}}{\rho^{6}}+2 \frac{x_{1} x_{2}}{\rho^{4}}, \\
\left(\frac{x_{2}^{3}}{\rho^{4}}\right)_{, 1}=-4 \frac{x_{1} x_{2}^{3}}{\rho^{6}}, & \left(\frac{x_{2}^{3}}{\rho^{4}}\right)_{, 2}=-4 \frac{x_{2}^{4}}{\rho^{6}}+3 \frac{x_{2}^{2}}{\rho^{4}}+\frac{3 \pi}{4} \delta(\boldsymbol{\rho}) .
\end{array}
$$

Further generalized derivatives can be taken similarly, leading to homogeneous generalized functions of degree -3 . Two results we need are

$$
\left(\frac{1}{\rho^{2}}\right)_{, \alpha}=-2 \frac{x_{\alpha}}{\rho^{4}}-\pi \delta_{, \alpha}(\boldsymbol{\rho})
$$




$$
\left(\frac{x_{\alpha} x_{\beta}}{\rho^{4}}\right)_{, \alpha}=-4 \frac{x_{\alpha} x_{\beta} x_{\gamma}}{\rho^{6}}+\frac{x_{\alpha} \delta_{\beta \gamma}+x_{\beta} \delta \gamma_{\alpha}}{\rho^{4}}-\frac{\pi}{4}\left(\delta_{\alpha \beta} \delta, \gamma(\boldsymbol{\rho})+\delta_{\beta \gamma} \delta, \alpha(\boldsymbol{\rho})+\delta_{\gamma_{\alpha}} \delta_{, \beta}(\boldsymbol{\rho})\right) .
$$

The latter equation written out in full is

$$
\begin{array}{rlrl}
\left(\frac{x_{1}^{2}}{\rho^{4}}\right)_{, 1} & =-4 \frac{x_{1}^{3}}{\rho^{6}}+2 \frac{x_{1}}{\rho^{4}}-\frac{3 \pi}{4} \delta_{, 1}(\boldsymbol{\rho}), & \left(\frac{x_{1}^{2}}{\rho^{4}}\right)_{, 2} & =-4 \frac{x_{1}^{2} x_{2}}{\rho^{6}}-\frac{\pi}{4} \delta_{, 2}(\boldsymbol{\rho}), \\
\left(\frac{x_{1} x_{2}}{\rho^{4}}\right)_{, 1} & =-4 \frac{x_{1}^{2} x_{2}}{\rho^{6}}+\frac{x_{2}}{\rho^{4}}-\frac{\pi}{4} \delta_{, 2}(\boldsymbol{\rho}), & \left(\frac{x_{1} x_{2}}{\rho^{4}}\right)_{, 2} & =-4 \frac{x_{1} x_{2}^{2}}{\rho^{6}}+\frac{x_{1}}{\rho^{4}}-\frac{\pi}{4} \delta_{, 1}(\boldsymbol{\rho}), \\
\left(\frac{x_{2}^{2}}{\rho^{4}}\right)_{, 1} & =-4 \frac{x_{1} x_{2}^{2}}{\rho^{6}}-\frac{\pi}{4} \delta_{, 1}(\boldsymbol{\rho}), & \left(\frac{x_{2}^{2}}{)_{, 2}}=-4 \frac{x_{2}^{3}}{\rho^{6}}+2 \frac{x_{2}}{\rho^{4}}-\frac{3 \pi}{4} \delta_{, 2}(\boldsymbol{\rho}) .\right.
\end{array}
$$

\section{Appendix C. Derivation of Static Fields of a Straight Defect from the General Theory}

In III we derived general expressions for the static fields in an infinitely extended isotropic body. We showed there that a condition on the plastic strain for the integrals to converge is that $e_{i j}^{P}(\mathbf{r})$ approaches zero faster than $r^{-1}$ as $r \rightarrow \infty$. This condition is not satisfied for straight defect lines, as can be seen from (3.3) and (4.7). However, it is still possible to find the functional dependence of the static fields from the general formulas by ignoring infinite terms that appear in the integrations. The correctness of the resulting static fields can then be assessed by checking them in the general field theory, as is done in the text.

The general expressions that we shall use are the following: For a dislocation line, the displacement, (III4.21),

$$
u_{i}^{T}(\mathbf{r})=\frac{1}{8 \pi} \int_{S}\left[R, j j k b_{k} d S_{i}^{\prime}+R_{, j k k} b_{i} d S_{j}^{\prime}-\frac{1}{1-\nu} R_{, i j k} b_{k} d S_{j}^{\prime}+\frac{\nu}{1-\nu} R_{, i j j} b_{k} d S_{k}^{\prime}\right],
$$

and the elastic distortion, (III4.23),

$$
\beta_{i j}(\mathbf{r})=\frac{b_{l}}{8 \pi} \oint_{L}\left[R_{, l n n} \epsilon_{i j k} d L_{k}^{\prime}+R_{, k n n} \epsilon_{i k l} d L_{j}^{\prime}+\left(\frac{R, i j k}{1-\nu}-\delta_{i j} R_{, k n n}\right) \epsilon_{k l m} d L_{m}^{\prime}\right] .
$$

For a disclination line with its axis through the origin, the displacement, (III6.10),

$$
u_{i}^{T}(\mathbf{r})=\frac{1}{8 \pi} \int_{S} \epsilon_{k m n} \Omega_{m} x_{n}^{\prime}\left[R_{j j k} d S_{j}^{\prime}+R_{j l l} \delta_{i k} d S_{j}^{\prime}-\frac{1}{1-\nu} R_{, i j k} d S_{j}^{\prime}+\frac{\nu}{1-\nu} R_{, i j j} d S_{k}^{\prime}\right],
$$

and the basic elastic fields, i.e., the elastic strain, (III6.11),

$$
\begin{array}{r}
e_{i j}(\mathbf{r})=\frac{1}{8 \pi} \oint_{L}\left[\epsilon_{l p q} \Omega_{p} x_{q}^{\prime}\left\{R_{, k n n}\left(\epsilon_{i k l} d L_{j}^{\prime}\right)_{(i j)}+\left(\frac{R_{i j k}}{1-\nu}-\delta_{i j} R, k n n\right) \epsilon_{k l m} d L_{m}^{\prime}\right\}+R,_{n n}\left(\Omega_{j} d L_{i}^{\prime}\right)_{(i j)}\right. \\
\left.+\left(\frac{R_{, i j}}{1-\nu}-\delta_{i j} R,,_{n n}\right) \Omega_{m} d L_{m}^{\prime}\right]
\end{array}
$$

and the elastic bend-twist, (III6.14),

$$
\kappa_{l j}(\mathbf{r})=\frac{1}{8 \pi} \oint_{L}\left[\epsilon_{k p q} \Omega_{p} x_{q}^{\prime} \quad\left\{R,,_{k l n n} d L_{j}^{\prime}-\frac{1}{2} R,{ }_{, j l n} d L_{k}^{\prime}\right\}-R,{ }_{k n n} \epsilon_{i k l} \Omega_{j} d L_{i}^{\prime}\right]
$$


where

$$
\begin{aligned}
& R=\left(X_{i} X_{i}\right)^{1 / 2}, \\
& X_{i}=x_{i}-x_{i}^{\prime} .
\end{aligned}
$$

The expressions (C1-5) are line and surface integrals. For the special geometry of figure 1 the curve $L$ is given by (A13)

$$
d L_{i}^{\prime}=\left(0,0, d L_{3}^{\prime}\right)=\left(0,0, d x_{3}^{\prime}\right), \mathbf{r}^{\prime}=\left(0,0, x_{3}^{\prime}\right) .
$$

Hence we have

$$
\begin{gathered}
R=\left(\rho^{2}+X_{3}^{2}\right)^{1 / 2}, \\
\rho=\left(x_{1}^{2}+x_{2}^{2}\right)^{1 / 2},
\end{gathered}
$$

by (B1). The surface $S$ is given by (A14)

$$
d S_{i}^{\prime}=\left(0, d S_{2}^{\prime}, 0\right)=\left(0,-d x_{1}^{\prime} d x_{3}^{\prime}, 0\right), \mathbf{r}^{\prime}=\left(x_{1}^{\prime}, 0, x_{3}^{\prime}\right)
$$

Hence

$$
R=\left(X_{1}^{2}+x_{2}^{2}+X_{3}^{2}\right)^{1 / 2}
$$

We shall first investigate the line integrals. All the necessary results can in general be derived from differentiating the basic integrals $\int R d L_{i}^{\prime}$ and $\int x_{i}^{\prime} R d L_{j}^{\prime}$. From (C8) the only non-vanishing components of these integrals are $\int R d L_{3}$ and $\int x_{3}^{\prime} R d L_{3}^{\prime}$. Since these integrals are along an infinite straight line they will diverge. So we first find the finite integral

$$
\begin{aligned}
\int_{-L+x_{3}}^{L+x_{3}} R d x_{3}^{\prime} & =\int_{-L}^{L} R d X_{3} \\
& =\left[\frac{1}{2} \rho^{2} \ln \left(X_{3}+R\right)+\frac{1}{2} x_{3} R\right]_{-L}^{L} \\
& =\rho^{2} \ln \frac{L+\left(\rho^{2}+L^{2}\right)^{1 / 2}}{\rho}+L\left(\rho^{2}+L^{2}\right)^{1 / 2}
\end{aligned}
$$

The integral we want is obtained when $L \rightarrow \infty$, but then the terms in (C13) containing $L$ will also approach infinity. However, these terms can be regarded as constants in the subsequent differentiations. Therefore they do not contribute to the fields and we can ignore them for practical purposes. So the functional dependence of the desired integral goes as

$$
\int_{L} R d L_{3}^{\prime} \rightarrow q,
$$

where we have defined the function

$$
q \equiv-\rho^{2} \ln \rho
$$

The justification for this procedure can also be given as follows: A sufficiently high derivative of (C13) will converge for $L \rightarrow \infty$ to a finite function of $\rho$. The same derivative of (C15) will give the 
same finite function. Now the relations $(\mathrm{Cl}-5)$ contain only derivatives of integrals such as $\int R d L_{i}$. Therefore we can use (C14-15) in them directly. We find the functional dependence of the other integral in the same way. First we find the finite integral

$$
\begin{aligned}
\int_{-L+x_{3}}^{L+x_{3}} x_{3}^{\prime} R d x_{3}^{\prime} & =\int_{-L}^{L}\left(x_{3}-X_{3}\right) R d X_{3} \\
& =\left[\frac{1}{2} x_{3} \rho^{2} \ln \left(X_{3}+R\right)+\frac{1}{2} x_{3} X_{3} R-\frac{1}{3} R^{3}\right]_{-L}^{L},
\end{aligned}
$$

and hence, comparing with (C13),

$$
\int_{L} x_{3}^{\prime} R d x_{3}^{\prime} \rightarrow x_{3} q .
$$

We now consider the surface integrals. All the necessary results can in general be obtained from $\int R d S_{i}^{\prime}$ and $\int x_{i}^{\prime} R d S_{j}^{\prime}$. From (C11) the only nonvanishing components are $\int R d S_{2}^{\prime}, \int x_{1}^{\prime} R d S_{2}^{\prime}$, and $\int x_{3}^{\prime} R d S_{2}^{\prime}$. The derivation of the first integral may be indicated as follows

$$
\begin{array}{r}
\int_{S} R d S_{2}^{\prime} \rightarrow-\int_{-L}^{L} \int_{-L}^{0} R d x_{1}^{\prime} d x_{3}^{\prime} \rightarrow \int_{-L}^{0}\left(X_{1}^{2}+x_{2}^{2}\right) \ln \left(X_{1}^{2}+x_{2}^{2}\right)^{1 / 2} d x_{1}^{\prime} \rightarrow \int_{x_{1}}^{L}\left(X_{1}^{2}+x_{2}^{2}\right) \ln \left(X_{1}^{2}+x_{2}^{2}\right)^{1 / 2} d X_{1} \\
=\left[\left(\frac{1}{3} X_{1}^{3}+X_{1} x_{2}^{2}\right) \ln \left(X_{1}^{2}+x_{2}^{2}\right)^{1 / 2}-\frac{2}{3} x_{2}^{3} \tan ^{-1} \frac{x_{2}}{x_{1}}-\left(\frac{1}{9} X_{1}^{3}+\frac{2}{3} X_{1} x_{2}^{2}\right)\right]_{x_{1}}^{L}
\end{array}
$$

Here the first result follows from the geometry of the surface $S$ in figure 1, the second by integration over $x_{3}^{\prime}$ which goes through the same as above but with $R$ given by (C12) instead of (C9), and the third by a change of variable. So the functional dependence becomes

$$
\int_{S} R d S_{2}^{\prime} \rightarrow g
$$

where we have defined the function

$$
g \equiv-\left(\frac{1}{3} x_{1}^{3}+x_{1} x_{2}^{2}\right) \ln \rho+\frac{2}{3} x_{2}^{3} \varphi+\left(\frac{1}{9} x_{1}^{3}+\frac{2}{3} x_{1} x_{2}^{2}\right)
$$

The next integral goes as

$$
\begin{aligned}
\int_{S} x_{1}^{\prime} R d S_{2}^{\prime} & \rightarrow \int_{-L}^{0} x_{1}^{\prime}\left(X_{1}^{2}+x_{2}^{2}\right) \ln \left(X_{1}^{2}+x_{2}^{2}\right)^{1 / 2} d x_{1}^{\prime} \\
& \rightarrow \int_{x_{1}}^{L}\left(x_{1}-X_{1}\right)\left(X_{1}^{2}+x_{2}^{2}\right) \ln \left(X_{1}^{2}+x_{2}^{2}\right)^{1 / 2} d X_{1} .
\end{aligned}
$$

This leads to the functional dependence

$$
\int_{S} x_{1}^{\prime} R d S_{2}^{\prime} \rightarrow h
$$


where we have defined the function

$$
h \equiv x_{1} g+\frac{1}{4} \rho^{4} \ln \rho+\frac{1}{16} x_{1}^{4}-\frac{1}{8} x_{1}^{2} x_{2}^{2} .
$$

The last integral goes as

$$
\int_{S} x_{3}^{\prime} R d S_{2}^{\prime} \rightarrow-\int_{-L}^{L} \int_{-L}^{0} x_{3}^{\prime} R d x_{1}^{\prime} d x_{3}^{\prime} \rightarrow x_{3} g
$$

The functions $q, g$, and $h$ depend only on $x_{1}$ and $x_{2}$. The derivatives of $q$ that we shall need are the following

$$
\begin{aligned}
& q_{, 11}=-2 \ln \rho-1-2 \frac{x_{1}^{2}}{\rho^{2}}, \\
& q_{, 12}=-2 \frac{x_{1} x_{2}}{\rho^{2}}, \\
& q_{, 22}=-2 \ln \rho-1-2 \frac{x_{2}^{2}}{\rho^{2}} \\
& q_{, \alpha \alpha}=-4(\ln \rho+1), \\
& q_{, 111}=-2 \frac{x_{1}}{\rho^{2}}-4 \frac{x_{1} x_{2}^{2}}{\rho^{4}}, \\
& q_{, 112}=-2 \frac{x_{2}}{\rho^{2}}+4 \frac{x_{1}^{2} x_{2}}{\rho^{4}}, \\
& q_{, 122}=-2 \frac{x_{1}}{\rho^{2}}+4 \frac{x_{1} x_{2}^{2}}{\rho^{4}}, \\
& q_{, 222}=-2 \frac{x_{2}}{\rho^{2}}-4 \frac{x_{1}^{2} x_{2}}{\rho^{4}}, \\
& q_{, 1 \alpha \alpha}=-4 \frac{x_{1}}{\rho^{2}}, \\
& q_{, 2 \alpha \alpha}=-4 \frac{x_{2}}{\rho^{2}}, \\
& q_{, 11 \alpha \alpha}=-4\left(\frac{1}{\rho^{2}}-2 \frac{x_{1}^{2}}{\rho^{4}}+\pi \delta(\boldsymbol{\rho})\right), \\
& q_{, 12 \alpha \alpha}=8 \frac{x_{1} x_{2}}{\rho^{4}}, \\
& q_{, 22 \alpha \alpha}=-4\left(\frac{1}{\rho^{2}}-2 \frac{x_{2}^{2}}{\rho^{4}}+\pi \delta(\boldsymbol{\rho})\right) .
\end{aligned}
$$

Here the Greek indices range only over 1 and 2. The results $(\mathrm{C} 25 \mathrm{k}-\mathrm{m})$ follow from (B8). The other derivatives we shall need are

$$
g_{, 112}=-2 \frac{x_{1} x_{2}}{\rho^{2}}
$$




$$
\begin{aligned}
& g_{, 122}=-2\left(\ln \rho+\frac{1}{2}+\frac{x_{2}^{2}}{\rho^{2}}\right), \\
& g_{, 222}=4 \varphi+2 \frac{x_{1} x_{2}}{\rho^{2}}, \\
& g_{, 1 \alpha \alpha}=-4(\ln \rho+1), \\
& g_{, 2 \alpha \alpha}=4 \varphi, \\
& h_{, 122}=-2 x_{1} \ln \rho+4 x_{2} \varphi-\frac{3}{2} x_{1}, \\
& h_{, 222}=6 x_{2} \ln \rho+4 x_{1} \varphi+6 x_{2}+\frac{1}{2} x_{2}, \\
& h_{, 1 \alpha \alpha}=-4 x_{1} \ln \rho+4 x_{2} \varphi-x_{1}, \\
& h_{, 2 \alpha \alpha}=4 x_{2} \ln \rho+4 x_{1} \varphi+6 x_{2}-\frac{1}{2} x_{2} .
\end{aligned}
$$

The displacement of the dislocation (C1) now reduces to

$$
u_{i}^{T}=\frac{1}{8 \pi}\left[g_{, \alpha \alpha \beta} b_{\beta} \delta_{i 2}+g_{, 2 \alpha \alpha} b_{i}-\frac{1}{1-\nu} g_{, i 2 \alpha} b_{\alpha}+\frac{\nu}{1-\nu} g_{, i \alpha \alpha} b_{2}\right]
$$

The individual components are

$$
\begin{aligned}
& u_{1}^{T}=-\frac{b_{1}}{8 \pi}\left[\frac{1}{1-\nu} g_{, 112}-g_{, 2 \alpha \alpha}\right]-\frac{b_{2}}{8 \pi}\left[\frac{1}{1-\nu} g_{, 122}-\frac{\nu}{1-\nu} g_{, 1 \alpha \alpha}\right], \\
& u_{2}^{T}=-\frac{b_{1}}{8 \pi}\left[\frac{1}{1-\nu} g_{, 122}-g_{, 1 \alpha \alpha}\right]-\frac{b_{2}}{8 \pi}\left[\frac{1}{1-\nu} g_{, 222}-\frac{2-\nu}{1-\nu} g_{, 2 \alpha \alpha}\right], \\
& u_{3}^{T}=\frac{b_{3}}{8 \pi} g_{, 2 \alpha \alpha},
\end{aligned}
$$

which give (3.12) by (C26), except for some trivial constants. The elastic distortion of the dislocation (C2) reduces to

$$
\begin{aligned}
& \beta_{\alpha \beta}=\frac{b_{\lambda}}{8 \pi}\left[\epsilon_{\alpha \beta} q_{, \lambda \nu \nu}+\epsilon_{\kappa \lambda}\left(\frac{q_{\alpha \beta \kappa}}{1-\nu}-\delta_{\alpha \beta} q_{, \kappa \nu \nu}\right)\right], \\
& \beta_{\alpha 3}=\frac{b_{3}}{8 \pi} \epsilon_{\alpha \beta} q_{, \beta \lambda \lambda} . \\
& \beta_{3 j}=0
\end{aligned}
$$

where $\epsilon_{\alpha \beta}$ is the two dimensional permutation symbol, i.e., $\epsilon_{11}=\epsilon_{22}=0, \epsilon_{12}=-\epsilon_{21}=1$. The nonvanishing components of (C30) are

$$
\begin{aligned}
& \beta_{11}=-\frac{b_{1}}{8 \pi}\left[\frac{q_{, 112}}{1-\nu}-q_{, 2 \nu \nu}\right]+\frac{b_{2}}{8 \pi}\left[\frac{q_{, 111}}{1-\nu}-q_{, 1 \nu \nu}\right], \\
& \beta_{21}=-\frac{b_{1}}{8 \pi}\left[\frac{q_{, 122}}{1-\nu}+q_{, 1 \nu \nu}\right]+\frac{b_{2}}{8 \pi}\left[\frac{q_{, 112}}{1-\nu}-q_{, 2 \nu \nu}\right],
\end{aligned}
$$




$$
\begin{aligned}
& \beta_{12}=-\frac{b_{1}}{8 \pi}\left[\frac{q_{, 122}}{1-\nu}-q_{, 1 \nu \nu}\right]+\frac{b_{2}}{8 \pi}\left[\frac{q_{, 112}}{1-\nu}+q_{, 2 \nu \nu}\right], \\
& \beta_{22}=-\frac{b_{1}}{8 \pi}\left[\frac{q_{, 222}}{1-\nu}-q_{, 2 \nu \nu}\right]+\frac{b_{2}}{8 \pi}\left[\frac{q_{, 122}}{1-\nu}-q_{, 1 \nu \nu}\right], \\
& \beta_{13}=\frac{b_{3}}{8 \pi} q_{, 2 \nu \nu} \\
& \beta_{23}=-\frac{b_{3}}{8 \pi} q_{, 1 \nu \nu}
\end{aligned}
$$

which give (3.14) by (C25). The displacement of the disclination (C3) reduces to

$$
\begin{aligned}
u_{i}^{T}=\frac{\Omega_{3}}{8 \pi}\left[h_{, 2 \alpha \alpha} \delta_{i 2}\right. & \left.-\frac{1}{1-\nu} h_{, i 22}+\frac{\nu}{1-\nu} h_{, i \alpha \alpha}\right]+\frac{\Omega_{i}}{8 \pi} \epsilon_{i j l} h_{, 2 \alpha \alpha} \\
& +\frac{\epsilon_{\alpha \beta} \Omega_{\beta}}{8 \pi}\left[x_{3} g_{, \alpha \gamma \gamma} \delta_{i 2}+x_{3} g_{, 2 \gamma \gamma} \delta_{i \alpha}-\frac{1}{1-\nu}\left(x_{3} g\right)_{, i 2 \alpha}+\frac{\nu}{1-\nu}\left(x_{3} g\right)_{, i \gamma \gamma} \delta_{\alpha 2}\right] .
\end{aligned}
$$

The individual components are

$$
\begin{array}{r}
u_{1}^{T=} \frac{\Omega_{1} x_{3}}{8 \pi}\left[\frac{1}{1-\nu} g_{, 122}-\frac{\nu}{1-\nu} g_{, 1 \gamma \gamma}\right]-\frac{\Omega_{2} x_{3}}{8 \pi}\left[\frac{1}{1-\nu} g_{, 112}-g_{, 2 \gamma \gamma}\right] \\
-\frac{\Omega_{3}}{8 \pi}\left[\frac{1}{1-\nu} h_{, 122}-\frac{\nu}{1-\nu} h_{, 1 \alpha \alpha}\right], \\
\begin{array}{r}
u_{2}^{T}=\frac{\Omega_{1} x_{3}}{8 \pi}\left[\frac{1}{1-\nu} g_{, 222}-\frac{2-\nu}{1-\nu} g_{, 2 \gamma \gamma}\right]-\frac{\Omega_{2} x_{3}}{8 \pi}\left[\frac{1}{1-\nu} g_{, 122}-g_{, 1 \gamma \gamma}\right] \\
-\frac{\Omega_{3}}{8 \pi}\left[\frac{1}{1-\nu} h_{, 222}-\frac{2-\nu}{1-\nu} h_{, 2 \alpha \alpha}\right],
\end{array}
\end{array}
$$

$u_{3}^{T}=\frac{\Omega_{1}}{8 \pi}\left[\frac{1}{1-\nu} g_{, 22}-\frac{\nu}{1-\nu} g_{, \gamma \gamma}\right]-\frac{\Omega_{2}}{8 \pi}\left[\frac{1}{1-\nu} g_{, 12}+h_{, 2 \alpha \alpha}\right]$,

which give (5.1) by (C26-27), except for some trivial linear terms. The elastic strain of the disclination (C4) reduces to

$$
\begin{aligned}
& e_{\alpha \beta}=-\frac{1}{8 \pi}\left[\Omega_{\gamma x_{3}}\left(\frac{q_{, \alpha \beta \gamma}}{1-\nu}-\delta_{\alpha \beta} q_{, \gamma_{\nu \nu}}\right)+\Omega_{3}\left(\frac{q_{, \alpha \beta}}{1-\nu}-\delta_{\alpha \beta} q_{, \nu \nu}\right)\right], \\
& e_{\alpha 3}=\frac{1}{8 \pi}\left[\Omega_{\alpha} q_{, \beta \beta}-\frac{1}{1-\nu} \Omega_{\beta} q_{, \alpha \beta}\right], \\
& e_{33}=0 .
\end{aligned}
$$

The individual components are

$$
e_{11}=-\frac{\Omega_{1} x_{3}}{8 \pi}\left[\frac{q_{, 111}}{1-\nu}-q_{, 1 \nu \nu}\right]-\frac{\Omega_{2} x_{3}}{8 \pi}\left[\frac{q_{, 112}}{1-\nu}-q_{, 2 \nu \nu}\right]+\frac{\Omega_{3}}{8 \pi}\left[\frac{q_{, 11}}{1-\nu}-q_{, \nu \nu}\right],
$$




$$
\begin{aligned}
& e_{22}=-\frac{\Omega_{1} x_{3}}{8 \pi}\left[\frac{q_{, 122}}{1-\nu}-q_{, 1 \nu \nu}\right]-\frac{\Omega_{2} x_{3}}{8 \pi}\left[\frac{q_{, 222}}{1-\nu}-q_{, 2 \nu \nu}\right]+\frac{\Omega_{3}}{8 \pi}\left[\frac{q_{, 22}}{1-\nu}-q_{\nu \nu}\right], \\
& e_{33}=0, \\
& e_{12}=-\frac{\Omega_{1} x_{3}}{8 \pi} \frac{q_{, 112}}{1-\nu}-\frac{\Omega_{2} x_{3}}{8 \pi} \frac{q_{, 122}}{1-\nu}+\frac{\Omega_{3}}{8 \pi} \frac{q_{, 12}}{1-\nu}, \\
& e_{23}=-\frac{\Omega_{1}}{8 \pi} \frac{q_{, 12}}{1-\nu}-\frac{\Omega_{2}}{8 \pi}\left[\frac{q_{, 22}}{1-\nu}-q_{, \nu \nu}\right], \\
& e_{31}=-\frac{\Omega_{1}}{8 \pi}\left[\frac{q_{, 11}}{1-\nu}-q_{, \nu \nu}\right]-\frac{\Omega_{2}}{8 \pi} \frac{q_{, 12}}{1-\nu},
\end{aligned}
$$

which give (5.5) by (C25), except for some trivial constant terms. The elastic bend-twist of the disclination (C5) reduces to

$$
\begin{aligned}
& \kappa_{\alpha \gamma}=\frac{\Omega_{\gamma}}{8 \pi} \epsilon_{\alpha \beta} q_{, \beta \nu \nu}, \\
& \kappa_{\alpha 3}=\frac{1}{8 \pi}\left[\Omega_{\gamma} x_{3} \epsilon_{\beta \gamma} q_{, \alpha \beta \nu \nu}+\Omega_{3} \epsilon_{\alpha \beta} q_{, \beta \nu \nu}\right], \\
& \kappa_{3 \gamma}=0 .
\end{aligned}
$$

The individual components are

$$
\begin{aligned}
& \kappa_{11}=\frac{\Omega_{1}}{8 \pi} q_{, 2 \nu \nu}, \\
& \kappa_{21}=-\frac{\Omega_{1}}{8 \pi} q_{, 2 \nu \nu}, \\
& \kappa_{31}=0, \\
& \kappa_{12}=\frac{\Omega_{2}}{8 \pi} q_{, 2 \nu \nu}, \\
& \kappa_{22}=-\frac{\Omega_{2}}{8 \pi} q_{, 1 \nu \nu} \\
& \kappa_{32}=0, \\
& \kappa_{13}=-\frac{\Omega_{1} x_{3}}{8 \pi} q_{, 12 \nu \nu}+\frac{\Omega_{2} x_{3}}{8 \pi} q_{, 11 \nu \nu}+\frac{\Omega_{3}}{8 \pi} q_{, 2 \nu \nu}, \\
& \kappa_{23}=-\frac{\Omega_{1} x_{3}}{8 \pi} q_{, 22 \nu \nu}+\frac{\Omega_{2} x_{3}}{8 \pi} q_{, 12 \nu \nu}-\frac{\Omega_{3}}{8 \pi} q_{, 1 \nu \nu}, \\
& \kappa_{33}=-\frac{\Omega_{1}}{8 \pi} q_{, 2 \nu \nu}+\frac{\Omega_{2}}{8 \pi} q_{, 1 \nu \nu},
\end{aligned}
$$

which give (5.9) by (C25). 


\section{References}

[1] de Wit, R., in Fundamental Aspects of Dislocation Theory (NBS Spec. Publ. 317), Ed. by J. A. Simmons, R. de Wit, and R. Bullough (U.S. Government Printing Office, Washington, 1970), p. 651. In this paper referred to as I.

[2] de Wit, R., J. Res. Nat. Bur. Stand. (U.S.), 77 A (Phys. and Chem.), No. 1, 49-100 (Jan.-Feb. 1973). In this paper referred to as II.

[3] de Wit, R., J. Res. Nat. Bur. Stand. (U.S.), 77A (Phys. and Chem.), No. 3, 359-368 (May-June 1973). In this paper referred to as III.

[4] Volterra, V., Annls. scient. Ec. norm. sup., Paris [3], 24, 400 (1907).

[5] Love, A. E. H., A Treatise on the Mathematical Theory of Elasticity, 3rd ed. (Cambridge U. P., 1920).

[6] Frank, F. C., Disc. Faraday Soc. 25, 19 (1958).

[7] Nabarro, F. R. N., Theory of Crystal Dislocations (Clarendon Press, Oxford, 1967).

[8] Gel'fand, I. M., and Shilov, G. E., Generalized Functions, Vol. I (Academic Press, New York, 1964).

[9] Mura, T., Arch. Mech. Stos. 24, 449 (1972).

[10] Anthony, K. H., Arch. Rat. Mech. Anal. 39, 43 (1970).

[11] Eshelby, J. D., Brit. J. Appl. Phys. 17, 1131 (1966).

[12] Li, J. C. M., Acta Met. 8, 563 (1960).

[13] Schober, T., and Baluff, R. W., phys. stat. sol. (b) 44, 115 (1971).

[14] de Wit, R., J. Appl. Phys. 42, 3304 (1971). 